\title{
A qualitative study on the effect of lymphatic filariasis on women: experiences, needs and implications for lymphedema management programs
}

Citation for published version (APA):

Person, B. (2008). A qualitative study on the effect of lymphatic filariasis on women: experiences, needs and implications for lymphedema management programs. [Doctoral Thesis, Maastricht University]. Datawyse / Universitaire Pers Maastricht. https://doi.org/10.26481/dis.20081016bp

Document status and date:

Published: 01/01/2008

DOI:

10.26481/dis.20081016bp

Document Version:

Publisher's PDF, also known as Version of record

Please check the document version of this publication:

- A submitted manuscript is the version of the article upon submission and before peer-review. There can be important differences between the submitted version and the official published version of record.

People interested in the research are advised to contact the author for the final version of the publication, or visit the DOI to the publisher's website.

- The final author version and the galley proof are versions of the publication after peer review.

- The final published version features the final layout of the paper including the volume, issue and page numbers.

Link to publication

\footnotetext{
General rights rights.

- You may freely distribute the URL identifying the publication in the public portal. please follow below link for the End User Agreement:

www.umlib.nl/taverne-license

Take down policy

If you believe that this document breaches copyright please contact us at:

repository@maastrichtuniversity.nl

providing details and we will investigate your claim.
}

Copyright and moral rights for the publications made accessible in the public portal are retained by the authors and/or other copyright owners and it is a condition of accessing publications that users recognise and abide by the legal requirements associated with these

- Users may download and print one copy of any publication from the public portal for the purpose of private study or research.

- You may not further distribute the material or use it for any profit-making activity or commercial gain

If the publication is distributed under the terms of Article 25fa of the Dutch Copyright Act, indicated by the "Taverne" license above, 


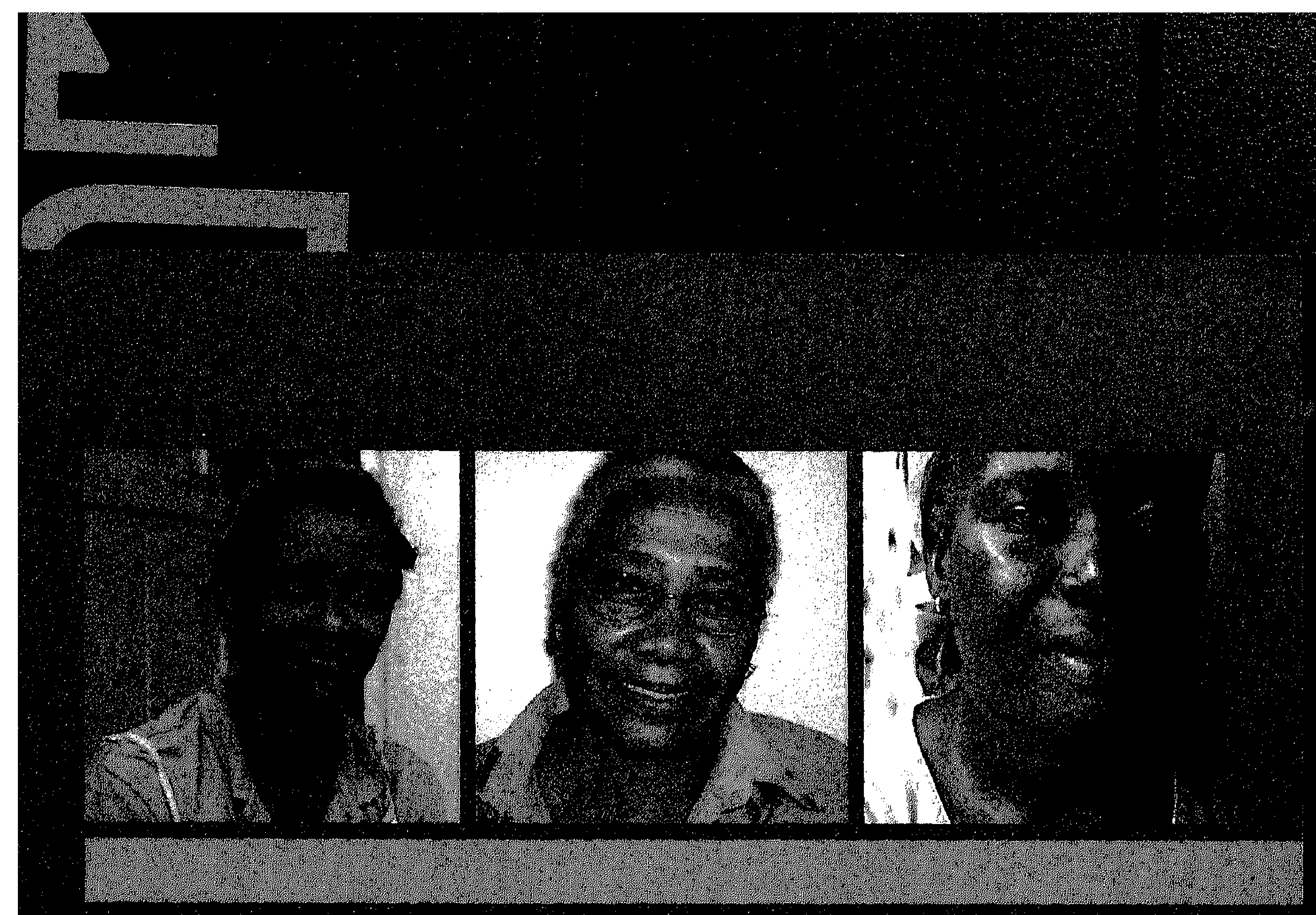

\section{A QUALITATIVE STUDY ON THE EFFECT OF LYMPHATIC FILARIASIS ON WOMEN}

Experiences, needs and implications for lymphedema management programs

Bobbie Person 
A qualitative study on the effect of lymphatic filariasis on women:

Experiences, needs and implications for lymphedema management programs

Bobbie Person 
ISBN: 9789052787596

Copyright: Bobbie Person, 2008

Printed by Datawyse, Universitaire Pers Maastricht 
A qualitative study on the effect of lymphatic filariasis on women: Experiences, needs and implications for lymphedema management programs

\section{DISSERTATION}

to obtain the degree of Doctor at the Maastricht University on the authority of the Rector Magnificus Prof.dr. G.P.M.F Mols, in accordance with the decision of the Board of Deans, to be defended in public on Thursday 16 October 2008, at 1400 hours

by

Bobbie Person

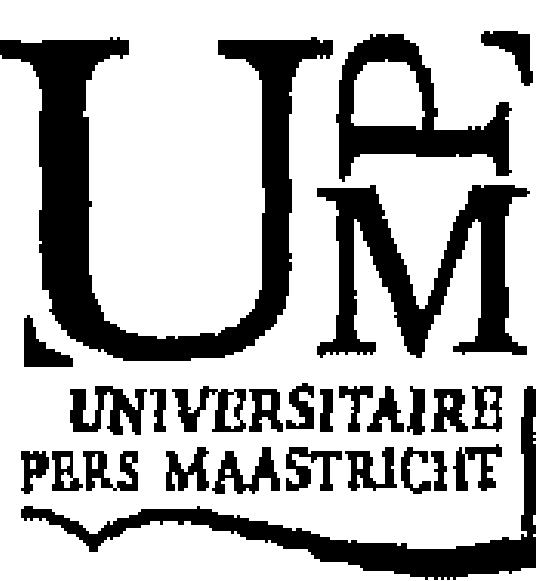




\section{Supervisor}

Prof. dr. H.W. van den Borne

\section{Co-supervisor}

Prof. dr. L.K. Bartholomew (University of Texas, Houston, USA)

\section{Assessment Committee}

Prof. dr. G.J. Kok (chairman)

Prof. dr. P. Kager (University of Amsterdam)

Dr. A. Krumeich

Prof. dr. H. Schaalma

Prof. dr. N.K. de Vries

CDC Advisor:

Dr. David Addiss (Centers for Disease Control and Prevention, Atlanta, Georgia, USA)

The studies presented in this dissertation were conducted under the auspices of the school for Public Health and Primary Care: CAPHRI at Maastricht University, The Netheriands. The school for Public Health and Primary Care: CAPHRl is part of the Netheriands School of Primary Care Research (CaRe), which has been acknowledged since 1995 by The Royal Netherlands Academy of Art en Sciences (KNAW).

\section{Funding and support}

Fieldwork for these studies was support by the Centers for Disease Control and Prevention (CDC) in Atlanta, Georgia, USA. Grants were provided by the Office of Minority and Women's Health (CDC).

\section{Disclaimer}

The opinions or assertions contained in this manuscript are the private ones of the authors and are not to be construed as official or reflecting the view of the US Public Health Service or Department of Health and Human Services. Use of trade names is for identification only and does not imply endorsement by the US Public Health Service or Department of Health and Human Services. 


\section{Dedication}

To all the women I met who shared their private lives with me.

Their determination to walk through life with wisdom, strength, and humor inspire me. 


\section{Table of Contents}

Chapter 1

General Overview

1. Purpose of the study

2. A general overview of lymphatic filariasis

3. Lymphatic filariasis in the Dominican Republic and Ghana

4. Study design

5. Major findings

6. Study limitations

7. Recommendations

Chapter 2

A Qualitative Study of the Psychosocial and Health Consequences associated with Lymphedema among Women in the Dominican Republic

Chapter 3

"Can It Be that God Does Not Remember Me?": A Qualitative Study on the Psychological

Distress, Suffering, and Coping of Dominican Women with Chronic Filarial Lymphedema and

Elephantiasis of the Leg

Chapter 4

Health-Seeking Behaviors and Self-Care Practices of Dominican Women with Lymphedema of the Leg: Implications for Lymphedema Management Programs

Chapter 5

Disrupted Social Connectedness among Dominican Women with Chronic Filarial Lymphedema

Chapter 6 . .81

Health-Related Stigma among Women with Lymphatic Filariasis from the Dominican Republic and Ghana

Chapter 7. 100

Evidence- and Theory-informed Recommendations for Program Planning to Improve Selfmanagement of Filarial Lymphedema in Women

Samenvatting

Acknowledgements

Biography 


\section{Chapter 1}

\section{General Overview}

This chapter presents the purpose of the study along with an overview of the life-cycle of the parasite that causes lymphatic filariasis, establishment of filarial infection, the resulting clinical manifestation of lymphedema among women, lymphatic filariasis as a global public health priority, and the epidemiology of filariasis-related disease in the Dominican Republic and Ghana. The study design and qualitative methods of inquiry and analysis are also described. The major findings along with study limitations and conclusions are presented. The chapter closes with recommendations derived from the study.

\section{Purpose of the study}

The clinical manifestations of lymphatic filariasis, a mosquito-transmitted disease, have been described throughout history. As early as $70 \mathrm{AD}$, the Indian textbook know as the Sushruta Samhita refers to a disease know as slipada (sli-elephant; padafoot) (1). Lymphatic filariasis is an infectious disease with global impact and clinical manifestations that range from asymptomatic damage to the lymphatic system to chronic grossly disfiguring lymphedema of the leg (Figure 1.1) (2-4). The preponderance of research on lymphatic filariasis in the literature is typically epidemiological studies on transmission and prevalence of disease (5-9). In-depth studies have characteristically been representative of men, particularly with hydrocele of the scrotum and lymphedema of the leg $(10-13)$. Data have noted significant psychosocial hardships, sexual dysfunction, reduced income and social status, stigmatization, and other cultural constraints imposed on men by the sequelae of disease $(13-15)$. Few studies have addressed the sequelae of lymphatic filariasis on

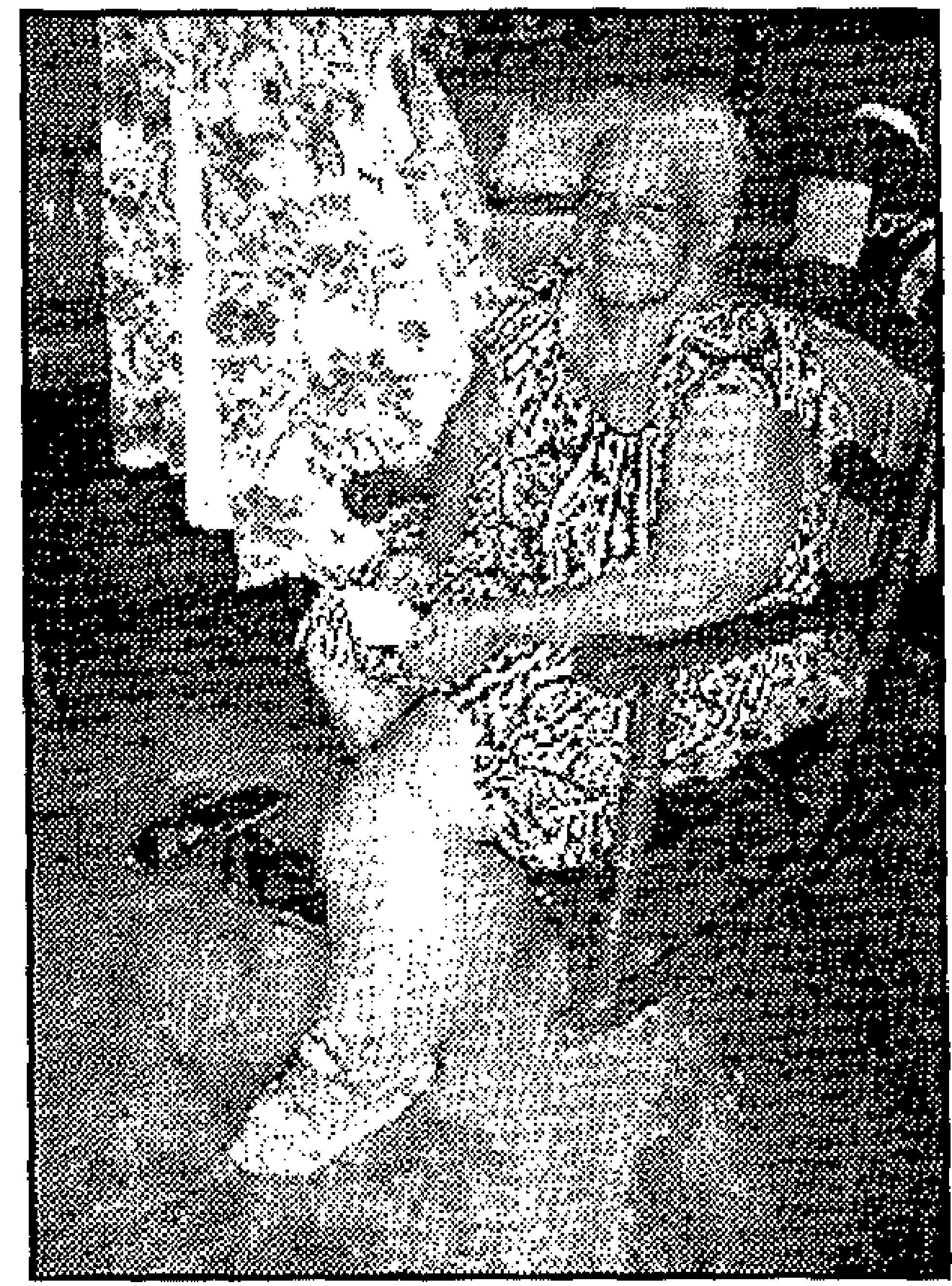

Figure 1.1. Woman with lymphedema of the leg. the quality of life of affected women who often face gender inequality in education and the labor market while carrying a disproportionate burden of responsibility for managing the household's childcare, water, sanitation, fuel, and waste management needs in resource-poor countries (16-25). Studies presented in this dissertation were derived from a two-year-long qualitative research investigation specifically into 
the lives of women with lymphatic filariasis in the Dominican Republic and Ghana (20-25).

The purpose of the study was to better understand the effect of lymphedema on the lives of women in resource-poor countries where lymphatic filariasis is endemic. The main emergent study themes of which the data provided greater understanding included 1) the psychosocial and health consequences of lymphedema; 2) the implications of lymphedema on psychological health; 3) women's health-seeking behaviors and self-care practices; 4) the role of social support in coping and care, 5) women's experience of health-related stigma; and 6) how to plan for prevention and control programs for early detection of disease, prevention of disease progression, and medical and behavioral management of sequelae of disease and resulting disability.

\section{A general overview of lymphatic filariasis}

\subsection{Lymphatic filariasis as a global public health priority}

Lymphatic filariasis is one of 13 parasitic and bacterial infections considered neglected tropical diseases $(3,26)$. Neglected diseases are some of the most common afflictions to humankind that affect more than 2.5 billion people that live on less than $\$ 2$ per day $(3,26)$. Lymphatic filariasis is caused by nematodes (roundworms) that live in the human lymphatic vessels and blood of humans $(27,28)$. This mosquitotransmitted parasitic disease affects over 120 million people and is estimated to be a leading cause of permanent disability worldwide (4,29-31). It is typically found in the tropics and subtropics of Africa, Asia, the Pacific Islands, and the Americas $(2,4,28,30,32)$. More than one billion people are at risk for infection in more than 80 countries and an estimated 44 million people, most of them living in poverty, are suffering from chronic symptomatic conditions such as lymphedema, hydrocele, chyluria, and episodic bouts of bacterial dermatolymphangioadenitis $(4,30-32)$. Researchers posit that the remainder of the 120 million affected people suffers from hidden damage to the lymphatic and renal systems that often go undiagnosed. Thirty-two of the 38 least developed countries in the world are endemic for lymphatic filariasis (3). Lymphatic filariasis is a disease of poverty which has a direct and indirect impact resulting in stigmatization, diminished productivity, and increased medical costs that promote even greater poverty.

In 1997, the World Health Assembly (WHA) recognized lymphatic filariasis as a potentially eradicable tropical disease and passed a resolution (50.29) that requested the member states of the World Health Organization (WHO) to commit to the global elimination of this disease $(33,34)$.

The World Health Organization (WHO), in 1997, also identified community management and care of chronic lymphedema or elephantiasis as one of the top twenty lymphatic filariasis research priorities (35). In 1998, WHO initiated the Global Program to Eliminate Lymphatic Filariasis (GPEFL), an initiative aimed at complete elimination of filariasis by 2020 (36). The program is based on two key strategies: interruption of transmission to prevent future disease and morbidity control for those suffering with clinical manifestations. 


\subsubsection{Interruption of transmission}

It has been demonstrated that the interruption of transmission of disease can be accomplished through the mass distribution of albendazole with either ivermectin or diethylcarbamazine to the entire eligible population once a year for five to six years in countries where disease is endemic (3). The pharmaceuticals kill the circulating larval form of the parasite, known as microfilariae (mf), and thereby reduce transmission, which benefits future generations. Some countries are also examining another way to interrupt transmission, the fortification of salt with diethylcarbamazine (37-41). Mass drug administration campaigns must continue for at least five years which requires political will, financial resources, public health infrastructure, and often technical support to be effective. The Dominican Republic is one of four countries in the Americas region that has an ongoing elimination program (5). In the Africa region, Ghana is also one of the countries with an ongoing program to interrupt transmission of the filarial parasite (3).

\subsubsection{Morbidity control}

The interruption of transmission of disease is a long-term solution for future generations. Even if transmission was interrupted and disease eliminated today there would still be millions of people suffering with the chronic clinical manifestations of disease since antifilarial drugs have little, if any, impact on established disease. Globally, countries are beginning to initiate national lymphedema management programs targeted to those who suffer chronic clinical manifestations of disease to complement mass drug distribution for interrupting transmission (3). The goal of treatment and care for the sequelae of disease are to prevent, reverse or halt progression of disease and resulting disability. Current lymphedema management control strategies include adequate health education, compliance with a regime of self-care that includes a medically prescribed hygiene and wound care intervention with exercise and elevation activities (42-47).

Prescribed lymphedema management strategies have provided a significant advance in the treatment of bacterial and fungal infections that promote disease progression and further compromise lymphatic function. These strategies have shown promising results in preventing and reducing dermatolymphangioadenitis, commonly called an acute attack, and anecdotally improving overall quality of life, alleviating pain and preventing further suffering (44-47). The tailoring of lymphedema management programs is often hindered by a lack of adequate information on gender-specific, cultural, psycho-social and quality of life (QOL) issues which influence the adoption of lymphedema management techniques and self-management practices. This dissertation focuses upon these issues with the intent of alleviating the suffering of women with clinical manifestations of disease rather than the interruption of transmission.

\subsection{Life cycle and establishment of infection}

The main mosquito vector for lymphatic filariasis found in the Dominican Republic is Culex quinquefasciatus, while in Ghana the main vector is the Anopheles gambiae (30). The parasite Wuchereria bancrofti (Figure 1.2) is a microscopic, 
thread-like roundworm responsible for bancroftian filariasis, which accounts for an estimated $90 \%$ of the lymphatic filariasis worldwide; it is the only known cause of lymphatic filariasis in the Dominican Republic and Ghana (30). India, Nigeria, Bangladesh, and Indonesia account for an estimated $70 \%$ of lymphatic filariasis worldwide $(30,48)$. Bancroftian filariasis occurs predominately in Africa, Asia, the Pacific Islands, and the Americas while Brugia malayi and B. timori Figure 1.2. Wuchereria bancrofti parasite. account for the remaining disease occur in Asia and some Pacific Islands $(30,48)$.

Humans are the only host for Wuchereria bancrofti. As shown in Figure 1.3, infected mosquitoes release parasitic filarial larvae when they take a blood meal from a human (27). These larvae migrate to the lymphatic vessels and lymph nodes. In approximately six to 12 months they develop into adult worms that mate and damage the lymphatic vessels and lymph nodes. The adult female worms reside in the lymphatic vessels for approximately six years, producing thousands of microfilaria which circulate in the blood. The microfilaria are taken up by mosquitoes during blood meals. Inside the mosquitoes, the microfilaria develop, in 1 to 2 weeks, into infective filariform (third-stage) larvae that are capable of initiating another cycle when the mosquito takes another blood meal. 


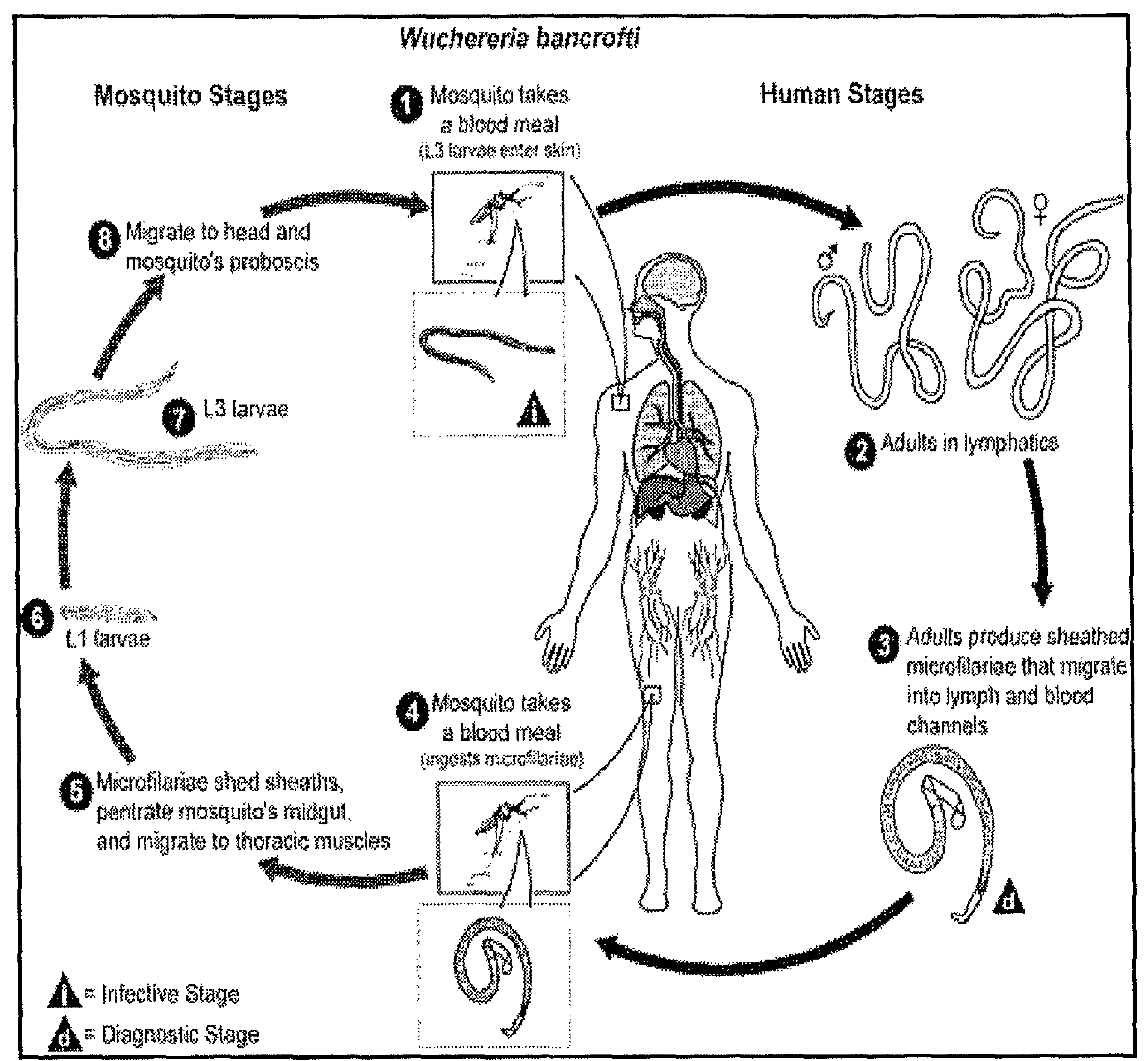

Figure 1.3. Lifecycle of Wuchereria bancrofti.

\subsection{Clinical manifestations associated with lymphatic filariasis}

Lymphatic filariasis infection is typically acquired in childhood although clinical manifestations may not present until many years later. Only a small percentage of infected individuals will present with clinical symptoms such as acute filarial lymphangitis, acute dermatolymphangioadenitis, and lymphedema (2). Most infected individuals will remain asymptomatic while being microfilariae positive, with possibly thousands of microfilariae in their blood and adult worms in the lymphatic system. Recent research posits that even without apparent clinical manifestations many people have hidden lymphatic pathology and renal involvement. Clinical manifestations present differently in different parts of the world depending, in part, upon the parasite involved. There are typically three distinct major clinical manifestations associated with lymphatic filariasis: acute inflammatory episodes, lymphedema, and hydrocele that have the attention of public health practitioners and researchers. In the late 1990s, two discrete acute 
inflammatory syndromes were identified by Gerusa Dryer and colleagues in Brazil (49). Acute filarial lymphangitis resulting from the death of adult worms (AFL) and acute dermatolymphangioadenitis (ADLA) the result of secondary bacterial infections are the two major acute inflammatory responses associated with lymphatic filariasis $(2,49-52)$. Male urogenital diseases associated with lymphatic filariasis, including testicular hydrocele (swelling due to excess fluid inside the tunica vaginalis), and swelling associated with lymphedema of the scrotal and penile skin, will not be addressed in this manuscript $(2,10)$. Chyluria, known as "milky urine," caused by leaking of lymph fluid into the urine, will also not be addressed (53). This section will be limited to the clinical manifestation of acute filarial lymphangitis, acute dermatolymphangioadenitis, and lymphedema which can be addressed through public health approaches in developing countries.

In a recent review article Addiss et al. noted that the sequelae of disease are currently believed to be dependent upon a wide range of factors such patient's age, gender, anatomical location of the adult worms, species of parasite, immune response, and secondary bacterial infections (2). Early studies suggested that adult worms contributed to an obstructive process in the lymphatic vessels impairing lymphatic function and producing clinical manifestations. Recent research suggests that the natural history of disease has a non-obstructive mechanism that contributes to lymphatic pathology and immunological responses earlier in life than has been previously noted and may play a role in the outcome of infection and disease later in life $(2,54,55)$.

\subsubsection{Acute inflammatory episodes: acute filarial lymphangitis (ADL)}

Acute episodes of local inflammation involving skin, lymph nodes and lymphatic vessels (lymphangitis) often accompany the chronic lymphedema without an injury or entry lesion in the skin. Some of these are caused by the body's immune response to the parasite $(15,50,55)$. A "string sign" may be visible in which the inflamed lymphatic vessel presents in a cord-like fashion in the limb and can actually be palpated. In the breast it may appear as a nodule.

\subsubsection{Acute inflammatory episodes: acute dermatolymphangioadenitis (ADLA)}

A skin lesion generally serves as a point of entry for bacteria resulting in acute dermatolymphangioadenitis among individuals with lymphatic filariasis (56-58). Typical clinical presentation of acute dermatolymphangioadenitis is that of high fevers, chills, swelling, pain, malaise, nausea and sometimes vomiting. The skin is often demarcated by inflamed edematous plagues and hyper-pigmentation. Blisters may also appear and skin exfoliation of the limb often occurs (56-58). While edema typically subsides after the acute attack, repeated episodes lead to chronic swelling or permanent lymphedema. In bancroftian filariasis the lymph nodes in the inguinal, axillary, and epitrochlear areas are most often involved in women. 


\subsubsection{Lymphedema}

In lymphatic filariasis a wide range of clinical manifestations are chronic among women, including lymphedema of the entire leg or arm (Figure 1.4), vulva, and breasts as a result of repeated acute attacks of dermatolymphangioadenitis (59). In bancroftian filariasis the entire leg or arm is usually involved. Skin changes are common in the affected limb and include disfiguring skin folds, hyperkeratosis or calloused layers of the skin, pigment changes, pachydermia or abnormal thickening of the tissues, chronic ulcerations, epidermal and sub-epidermal nodules, hair growth, and dermatitis occurring between folds of the skin (59). Fungal infections are also common. Elephantiasis is the most advanced form of chronic lymphedema, and is characterized by severe fibrosis or thickening of the skin,

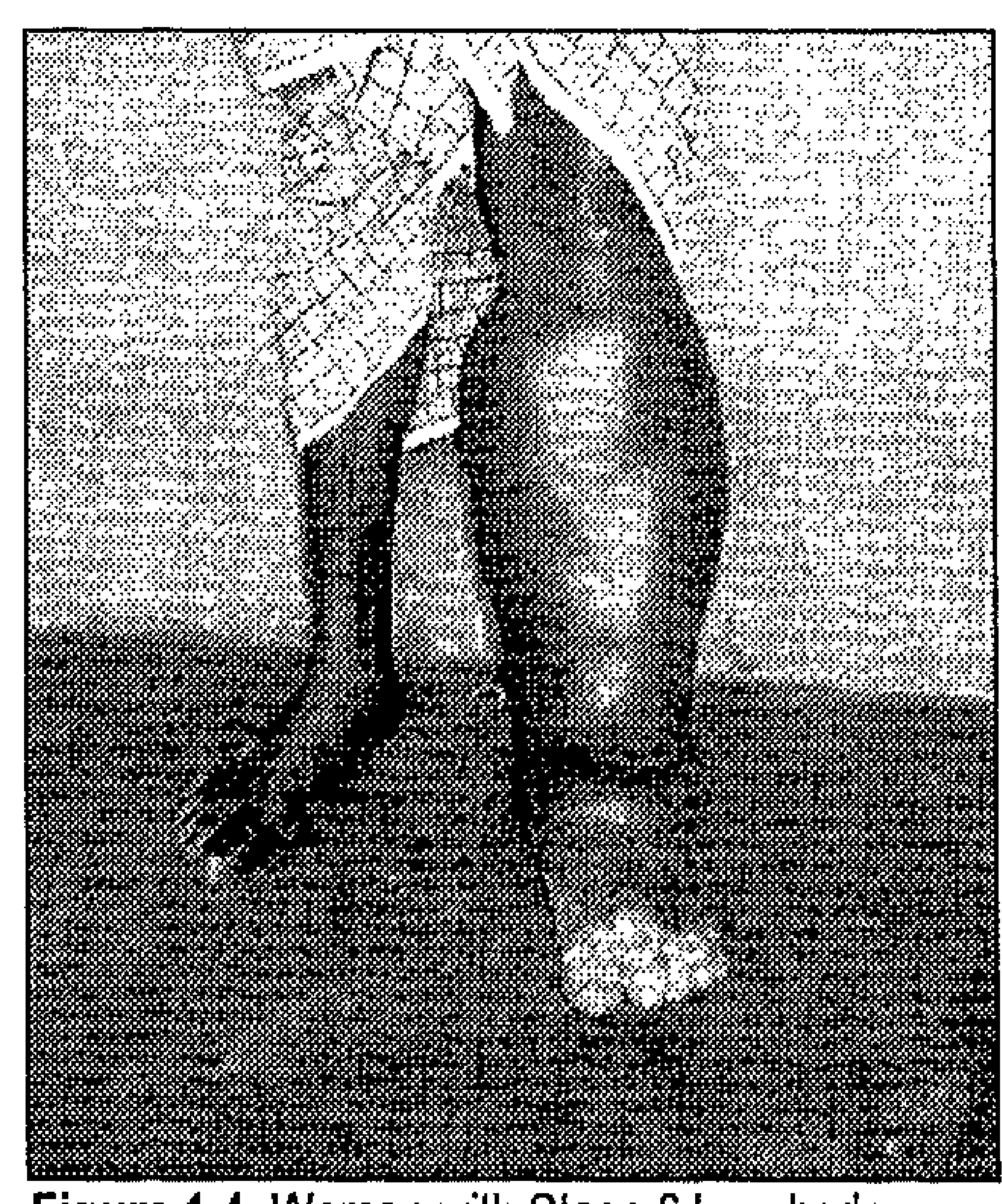

Figure 1.4. Woman with Stage 6 lymphedema. which makes it resemble the hide of an elephant. Tissues affected by lymphedema become highly susceptible to bacterial infection. The increased understanding of the role of bacterial infection in the progression of disease has contributed to improved treatment regimes.

\subsection{Staging algorithm severity of disease}

While researchers and medical practitioners have attempted to qualify the progression of lymphedema into discernible classifications there is no current global standard. The work of Dreyer et al. in developing lymphedema management guidelines proposes a staging procedure which classifies the lymphedema into specific stages which are linked to a corresponding set of clinical management strategies (60). Staging the lymphedema of the leg assists in providing patients the correct management techniques and determining potential disease progression. Based on the staging criteria of Dreyer et al., Stage 1 is reversible swelling that increase during the day and returns to normal at night with no specific treatment; Stage 2 is nonreversible swelling that does not change without lymphedema management techniques; permanent (non-reversing) lymphedema is considered Stage 3 if shallow skin folds are present; Stage 4 if there are protrusions or "knobs"; Stage 5 if deep skin folds are present; Stage 6 if "mossy lesions" are present; and Stage 7 if the patient is unable to perform activities of daily living $(42,60)$.

\subsection{Lymphatic filariasis diagnosis}

Historically, diagnosing filarial infection has been cumbersome due to the periodicity of microfilaria in the blood. In most parts of the world, the microfilariae have a nocturnal periodicity whereby the greatest concentration in the blood is 
around midnight-the peak mosquito feeding time. Typically, filarial infection is determined by detecting microfilariae on a blood slide. Recently, a simple "card test" which uses finger-prick blood droplets taken anytime during the day has revolutionized diagnosis (61). This immunochromatographic card test (ICT) is easy to use, provides results in approximately $5^{-15}$ minutes, is sensitive and specific, convenient, and at a reasonable cost (61). There is currently no laboratory test to actually confirm that lymphedema is filarial in origin (2).

\subsection{Lymphatic filariasis and women}

Increasingly, attention is being paid to promoting gender specific strategies for recruiting women into tropical disease prevention and control programs. While the magnitude of disease related to filarial lymphedema of the leg is significant among women in developing countries, little specific research has been conducted to explore and better understand either the effect of the condition on their daily lives or the effect of lymphedema management on the improvement of their lives (16-25).

\section{Lymphatic filariasis in the Dominican Republic and Ghana}

Wuchereria bancrofti, one of the parasites that cause lymphatic filariasis, was thought to have been brought to the Dominican Republic with forced migration of slaves from Africa in the 1600 s (5). Night-biting Culex Quinquefasciatus mosquitoes that live in polluted water are the primary vectors of Wuchereria bancrofti, thriving on unsanitary environmental conditions (62). Currently, approximately 740,000 people live in filarial-endemic areas in the Dominican Republic (63). In 2002, the prevalence of lymphedema nationwide was estimated to be $0.70 \%$ and prevalence of hydrocele to be $0.16 \%$ (64). Recent studies have found prevalence rates ranging between $2 \%$ and $14 \%$ for blood surveys, and $9 \%$ and $35 \%$ using the rapid immunochromatographic (ICT) card test (64). The highest infection rates occur in provinces in the southern region, where this study was conducted. In 2000 the Ministry of Health (MOH), partnering with the Centro Para el Control de Enfermedades Tropicales (CENCET), established a national program for the elimination of lymphatic filariasis.

Globally, one-third of those with lymphatic filariasis live in Africa and an estimated 500 million Africans are at risk for disease $(63,65)$. The $A$. gambiae and $A$. melas mosquitoes are the vectors for the parasite $W$. bancrofti, which causes lymphatic filariasis in Ghana (6). Almost half of the population of Ghana, 10.2 million people in 61 out of 138 districts, is estimated to be at risk for lymphatic filariasis $(6,63)$. In 1990, the first population-based survey of elephantiasis conducted in northern Ghana and showed that 735 out of $5846(12.6 \%)$ of the compounds visited had a least one person with reportable elephantiasis of the leg (64). In northern Ghana in a rural filarial endemic community $41 \%$ of the population over ten years of age had $W$. bancrofti microfilariae detected in the blood and $3 \%$ had chronic disease (66). The Ministry of Health has had a commitment to the elimination of lymphatic filariasis for more than 15 years (6). 


\section{Study design}

\subsection{Study area and prticipants}

This study was conducted in filariasis-endemic areas of the southern coastal region of the Dominican Republic (DR) on the Caribbean island of Hispaniola and in the rural fishing villages and farming communities in the southern coastal area of the Western and Central regions of Ghana, West Africa (67-70).

Using purposive sampling we recruited women for in-depth interviews who had moderate to severe lymphedema of the leg (stages $3-7$, based on the staging criteria of Dreyer and colleagues), varied in age and occupations, not routinely engaging in medically prescribed lymphedema self-management activities; and expressing willingness to be interviewed, audio-taped, and photographed $(42,71)$. Public health officials had recently conducted a combined health education campaign and mass drug distribution to interrupt disease transmission in filariasis-endemic regions of both countries. Clinic staff, ministry of health staff, and local communicable disease control officers referred women with visible edema of the leg for screening to determine cause of condition and stage of lymphedema if appropriate. The first 30 women in the DR and Ghana who met the study criteria were referred for an in-depth interview. Some women had no history of care while others had sought traditional and/or western medical treatments for their condition in the past. A few women had recently sought care receiving instruction in lymphedema management techniques but were not consistent or long-term performers of prescribed self-care. Of the 60 women recruited for an interview, four women did not have filarial lymphedema; there was one incomplete interview, damage to one interview tape, and two women declined to participate.

Additionally, 23 Ghanaian women were recruited from public health clinics supported by the Ministry of Health in the coastal towns of Princess Town and Winneba for 3 FGDs and 26 Dominican women from the Instituto Dermatológico de Cirugia y Piel (IDCP) in the coastal towns of Santo Domingo and Barahona for 3 FGDs. In both countries women were recruited for FGDs from clinic scheduling rosters and records, which included some women who were current clinic patients practicing medically prescribed self-management training for care of their leg and some who were new patients which offered some contrasting experiences reflecting on the role of the new program in influencing the typical care and treatment of lymphedema among women. Women were recruited during the two-week period prior to conducting the FGD. Those who met the study criteria were invited to participate in a FGD. The first 30 women who agreed were accepted into the study in each country. In Ghana we also conducted a Photovoice project where we gave cameras to women to document and discuss the day-to-day reality of living with their lymphedema.

\subsection{Ethical review}

Institutional and ethical review boards from the US Centers for Disease Control (CDC), the Centro Para el Control de Enfermedades Tropicales (CENCET), and the Ministry of Health for Ghana approved this study. Informed consent was 
read out loud and then signed with a signature or thumbprint by every participant. All women shown gave permission for their photographs to be reproduced for academic and educational purposes.

\subsection{Design and data collection}

Qualitative data were gathered through 52 in-depth interviews; 6 FGDs; field notes; and photographs of women in clinic, community, and home settings. Photographs were taken to allow study team to confirm stage of lymphedema in the leg. Data collection was facilitated by brief interview and moderator guides translated by bilingual research assistants into local languages of Spanish in the DR and Fanti in Ghana. Questions and probes in the guides were pretested with women with lymphedema in both countries to assure correspondence with local dialect, cultural meanings, and women's understanding. Interviews were conducted over 2 years to allowing for preliminary data analysis to help guide subsequent interviews. The 52 interviews, 2-3 hours long, took place locally in women's homes, clinics, and outdoor settings. Data saturation, the point at which no new themes were emerging, was achieved at 24 participants in both countries. FGDs were conducted to corroborate interview data, develop theme interpretation, explore concepts derived from individual interviews for greater details, and identify any contradicting information. FGDs were conducted in clinic and community settings. Following each interview and FGD the primary investigator taught a lymphedema self-management regimen that included demonstrations of leg washing, elevation, and exercise, a gift of local soap, and referral to local health care staff for follow-up.

\subsection{Data management and analysis}

Interviews and FGDs were audiotaped, transcribed verbatim, translated into English by a bilingual research assistant, and entered as a Microsoft Word document into ATLAS-ti to facilitate data coding, text searching, and analysis $(71,72)$. Transcripts were examined for emerging codes, concepts, categories, and themes $(72,73)$. The primary author and two research assistants derived coding schemes independently on a subset of interviews and then developed consensus on the codes. Independent coding on another subset of interviews was conducted to ensure consistency in implementing the coding strategy. Analyses drew upon Strauss and Corbin's explanation of open coding, axial coding and selective coding for analyzing the narratives and FGD transcripts (73-75). Open coding, a word by word analysis, was used to identify, name and categorize events and explanations found in the text. Open coding was followed by axial coding requiring the analyst to relate themes and categories with their subcategories to identify a more complex analysis of causal relationships in the data. Finally, selective coding was used to integrate and corroborate the emerging relationships and to construct the explanatory models related to the topic of interest (74). 


\section{Major findings}

The purpose of the study was to better understand the effect of lymphedema on the lives of women in developing countries where lymphatic filariasis is endemic. The main emergent study themes of which the data provided greater understanding included 1) the psychosocial and health consequences of lymphedema; 2) the implications of lymphedema on psychological health; 3) women's health-seeking behaviors and self-care practices; 4) the role of social support in coping and care; and 5) women's experience of health-related stigma. These findings provided evidence for the development of theory-based recommendations for program planning for early detection of disease, prevention of disease progression, and medical and behavioral management of sequelae of disease and resulting disability among women in developing countries were lymphatic filariasis is endemic.

We were able to gain insight in to the dynamic psychosocial and health consequences associated with lymphedema as reported by Dominican women in our study. Women derived their own explanatory model of illness, whereby they appraised and interpreted their symptoms within the experiences and contextual factors of their culture and daily lives. Women described an initial problem-solving phase in their explanatory model of illness where family and friends acted as lay health advisers providing health referrals to ensalmadores, curanderos, and brujos. Further exploration of this finding indicated that family, friends, and strong cultural beliefs influenced women's explanatory model of illness (EMS) and significantly influenced their health-seeking behaviors. Physical and functional limitations were not always associated with severity of symptoms or, as described in this study, stage of lymphedema. Despite functional limitations and psychological losses, many Dominican women were hesitant to consider themselves disabled. The most prominent consequence described by Dominican women was the ubiquitous state of psychological distress experienced by women across all stages of disease. While the sequelae of disease often resulted in a modification or loss of an occupational role, some women with limited and severe disease continued to care for themselves and their families, maintain income-generating activities, and even plan for new occupations. Religious beliefs, a positive outlook and hope provided the basis for positive future expectations while aging, weariness and hopelessness reinforced negative future expectations.

In using modified precepts of grounded theory methods, the psychological states of Dominican women and the coping strategies they used to ameliorate the detrimental psychological effects of this condition emerged as a critical finding. While some variation was expressed, women described feelings of embarrassment and shame; sadness, depression, and fear; personal losses; pity and social isolation; stigma; and despair which for some women led to suicidal thoughts. Women were also self-consciousness, ill ease, and ashamed about the appearance of their leg(s). Their inability to wear shoes prevented them from attending social activities and fulfilling expected cultural roles and responsibilities. The disfigurement of the leg also negatively influenced body image, self-esteem, and social identity among many women and was a source of stigmatization. The inability to meet the traditional Latin cultural roles for women as wife, mother, and nurturer often contributed to women feeling socially isolated which in turn led to an overall loss of purpose in life and 
lower self-esteem. Psychological distress was ubiquitous in the lives of women and the level, type, and amount of distress appeared to vary over time. Aging coupled with psychological distress, disrupted social connectedness, progressing disease appeared to create a highly vulnerable population of women. Internal resources of personality and coping methods and external resources of money, education, and social support appeared to influence coping abilities. While many women described meaningful experiences and adaptive coping strategies for dealing with their distress, it would be a mistake to underestimate the degree of suffering that they experienced.

Health-seeking behaviors and self-care practices of Dominican women were robustly discussed. As indicated earlier family, friends, and strong cultural beliefs influenced women's explanatory model of illness (EMS) and their health-seeking behaviors. Traditional healthcare providers and indigenous healers played an integral part of physical, mental, and spiritual healthcare for many women. Traditional healers often suggested specific procedures, incantations, generic potions, creams, injections and prayers for lymphedema. The current cultural paradigm of seeking indigenous healers as a first-line treatment often resulted in not only a significant delay in appropriate medical treatment but resulted also in potentially harmful treatments that could exacerbate the condition. It was often only the failure of these traditional methods of care that resulted in women seeking care by a trained healthcare provider. Even when seeking care from a trained physician, women were often misdiagnosed and provided only limited relief. A poor understanding of the disease, high healthcare costs, and frequent misdiagnosis by physicians led many women to eventually self-administer a regime of antibiotic injections and oral painkillers without medical consultations. There was also an extensive use of injectable and topical antibiotics by indigenous healers.

The role of social support in coping and care among women was addressed by women. An explanatory model of disrupted social connectedness was derived from Dominican women's reports on the usefulness and breakdown of their social relations and detailed the primary processes that caused social disconnectedness including: disease progression, women's failed attempts to adhere to cultural scripts, overtaxed social support resources, strained social interactions, damaged social roles in the community, and consequences of aging. The richness of their narratives allowed us to create a much more detailed picture of the effects of lymphedema on social connectedness than has been previous reported. Findings highlight the complexity of the life and disease management challenges faced by chronically ill women with lymphedema who lead restricted lives, experience a discredited sense of self, and exist in social isolation. Support from family and friends, particularly other females, and the church were particularly valued by women. Chronic lymphedema, aging, and disability, interacting with other medical conditions, often diminished social interactions, elicited exclusionary behaviors, decreased sense of self worth, and appeared to contribute to the emergence of depressive symptoms among women, which decreased their quality of life. The outcomes of disrupted social connectedness that emerged from our data included disrupted social support, poor health outcomes, loss of sense of self, psychological distress, depressive symptoms, and social isolation and exclusion. 
Experiences of health-related stigma were explored more in-depth through interviews with women with lymphatic filariasis in the Dominican Republic and Ghana. The overlapping constructs of enacted, perceived and internalized stigma provided a framework for observing a cycle of deleterious stigmatization experienced by women in both cultures. Women in both countries described the fear of contagion and personal discomfort related to the smell, sores, and physical disfigurement of their leg as the main contributor to stigmatization by others. Enacted stigma often resulted in a loss of income, loss of a social identity, decreased social interactions and access to resources, and labeling with further stigmatization. These enacted stigma experiences often led to perceived and internalized stigma contributing to increased fear, anxiety, depression, and suffering along with amplified self-isolating, selfdemeaning, and sick role behaviors, impaired body image, and in some cases suicidal thoughts. Women described management strategies, both negative and positive, influenced by personal and societal factors as contributing to negative health and psychosocial outcomes associated with living with this condition. Ghanaian women felt that their inability to work and being a contributing member of society was often their greatest loss. Dominican women described the loss of their sense of normalcy in appearance and ability to be a social person as their greatest loss. Drawing upon findings from the formative research, I posit that the three stigma constructs transcend the two cultures, have some underlying characteristics that overlap, and amplify the problems that women experience as the disease progresses, resources dwindle, and women age.

Study findings informed evidence- and theory-based recommendations for program planning to improve self-management of filarial lymphedema in women in developing countries. If disease transmission was completely interrupted today there would still be thousands of people living with health and psychosocial consequences of lymphatic filariasis for years to come. Limited funding for lymphedema management programs demands that health education prevention and promotion programs be deemed efficacious through the use of multiple theoretical and practical approaches that have been demonstrated to work. The Intervention Mapping framework as we have presented it allows for a problem-based tailored approach integrating theory with practical strategies for changing behaviors and environmental conditions in real-life situations to prevent disease progression, disability and further disfigurement.

\section{Study limitations}

In examining the specific implications of lymphatic filariasis sequelae on women in resource-poor countries problems did arise and study limitations do exist. There are several specific weaknesses in this qualitative study as explained in the next 4 sub-paragraphs.

\subsection{Funding}

The qualitative study would have been stronger if not constrained by funding limitations. Greater funding would have allowed for a study which included more women. In addition greater funding could have allowed for a study to include both men and women with lymphatic filariasis providing a more robust gender analysis of 
the impact of the sequelae of disease. An extended observational study component in which we could have observed daily life of women over time would have strengthened the self-reported experiences that were collected and analyzed in the study. Additionally, a formalized set of community interviews would have provided a comparison or contrasting of women's views with those of community members. The study would also have been stronger if study findings could have been translated into a quantitative survey administered to a representative sample of women with lymphatic filariasis to test the generalizability of our findings. Limitations in funding precluded the inclusion of such study components.

\subsection{Participants}

The women interviewed were from filariasis-endemic, peri-urban and urban communities in the southern coastal region of the Dominican Republic on the Caribbean island of Hispaniola and in the rural fishing villages and farming communities in the southern coastal area of the Western and Central regions of Ghana, West Africa. Social desirability bias and cultural factors unknown to the primary authors may have influenced the responses of some women.

Caution is suggested when considering these findings in relation to women with less education and fewer resources. Interviews were conducted in clinics, communities, and home settings. Bias related to the setting where the interview was conducted may occurred and would have been more apparent with greater numbers of interviews. In addition, there may have been women housebound due to advanced disease and disability that were not offered the opportunity to participate in the study or chose not to participate in the study thus our findings may not reflect the experiences of the most severely disfigured and disabled. We attempted to interview women with early stage lymphedema and found that their lymphedema experiences did not affect their daily lives and thus choose to focus upon women with observable permanent, lymphedema which in some way affected their day-to day life and plans for a future.

The loss of eight interviews due to interviewee drop-out and a damaged tape reduced our proposed participant sample size from an expected sixty interviewees to fifty-two across the two countries. While data saturation was noted at twenty-four interviews in each country, greater funding and time would have allowed us to replace those lost and the opportunity to explore any new issues which may have arisen.

\subsection{Methodological considerations}

Methodological limitations related to researcher bias, descriptive validity, interpretive validity, theoretical validity, internal and external validity also existed.

While debate exists on the use of the term validity in qualitative research there is agreement that qualitative research findings should be plausible, creditable, trustworthy, and therefore defensible $(75-76)$. Recently, some researchers have suggested measures of validity in qualitative research that we will present below for considering methodological limitations of this study (75-78). 
Researcher bias, in which a researcher finds what they set to find, can be a major threat to validity or creditability in qualitative research. While the primary author is a social scientist and has previous experience in qualitative research and working in resource-poor countries in cross-cultural settings, the dearth of literature on women with lymphatic filariasis and the devastating disfigurement that often results from this disease could contribute to an expectation of extreme suffering by women. Qualitative research is inductive and interpretative thus the analysis may be biased by the experience of the primary author and in-country researchers and their conceptual understanding of the culture of participants enrolled in the study. Attempts to control for researcher bias included an awareness of and ongoing reflection of preconceived ideas and beliefs throughout the study; inclusion of other researchers in discussions related to study design, implementation and interpretation of findings; and inclusion of and further exploration of those findings that were inconsistent with expectations and explanations, such as acknowledging women who do not feel stigmatized or who do not suffer from low self-esteem as a result of severe lymphedema (Chapter 6).

Descriptive validity refers to the factual accuracy of the researcher reporting descriptive information (i.e. what they saw and heard) (76). The primary author was not a native speaker of the multiple languages and dialects of women who were interviewed for the study and thus depended upon experienced Dominican and Ghanaian social scientists and research assistants for expressing and interpreting the nuances of dialect during interpersonal exchanges with women. While all interviews were taped, factual inaccuracies may have occurred during translation and transcription compromising descriptive validity. Translation during interviews, debriefing sessions following interviews and observations and review of transcriptions with tapes and corresponding translations were conducted to minimize threats to descriptive validity.

Interpretive validity represents the degree to which the participants' viewpoints, thoughts, feelings, intentions, and experiences are actually understood by the qualitative researcher (76). Interpretive validity is associated with accurate portrayal of the meaning and understanding attached to the topic under study by the person being interviewed. Attempts to control for threats to interpretive validity included the use of "member checking" or presenting the findings back to some women themselves to see if our interpretations were accurate; presenting our findings to other health care and public health professionals in the field who work with women to see if they were consistent with the thoughts and feelings that women had expressed to them; and to use verbatim quotes when writing up our findings so that the readers themselves can experience the actual language, dialect and personal meanings as expressed by women themselves (77).

Theoretical validity is the degree to which the data credibly fits with the theoretical explanation developed from the qualitative study. The study drew upon modified precepts of grounded theory where the researcher begins with the data and uses the data to generate a theory. In generating a theory in this study (Chapters 5 and 6) we may have developed relational constructs drawn upon faulty theoretical constructs or constructs lacking reliability in culturally diverse and resource-poor settings to explain the our phenomenon of interest. Attempts to control for threats to 
theoretical validity included the use of peer review whereby we presented and discussed with colleagues our theoretical explanations and received critical feedback; inclusion of and further exploration of those findings that were inconsistent with expectations and prevailing theoretical explanations; and triangulation of the data by drawing upon previous research related to lymphatic filariasis as well as previous behavioral and social science on the emergent topics of interest (e.g., social disconnectedness, stigma). While data saturation was obtained at 24 interviews in each country, a greater number of interviews and extending time in the field could promote stronger theoretical validity.

Internal validity in qualitative research is often associated with rigor and credibility or the degree to which a researcher is justified that a proposed relationship is casual or that it accurately reflects reality (76). The qualitative researcher recognizes that there may be multiple realities and attempts to represent these multiple realities adequately. Attempts to control threats to credibility or internal validity included the use of "member checking" or presenting the findings back to some women themselves to see if our interpretations were accurate; the use of field notes; consistent interviewing standards across interviews; triangulation of data and other strategies mentioned earlier in this section on descriptive, interpretive, and theoretical validity.

External validity is associated with the idea of generalizability of findings to other people, settings, and times (76) As typically noted in qualitative research, findings from this study conducted among a fairly small number of women may not be generalizable to broader global populations of women with this condition. The findings may though provide insight to health care and public health professional working with women with similar conditions, situations, and settings in resourcepoor countries.

\subsection{Unforeseen circumstances}

Unforeseen circumstances associated with government travel restrictions and funding cuts prohibited formal discussion with family members and traditional healers and delayed in-depth analysis of data from Ghana. In grounded theory a preliminary review of data from key informant interviews and focus group discussions inform subsequent interviews and focus group discussions so the primary author was familiar with the Ghana data but was unable to conduct a more comprehensive analysis until the end of the dissertation. The delay of in-depth analyses resulted in combined country data analyses for only two of the six manuscripts in the dissertation. Analysis of key informant interviews and focus group discussion data from Ghana was conducted and combined with field notes on informal discussions with family members, community members, health care and public health professionals for manuscripts in Chapter 6 on women's experiences of health-related stigma and Chapter 7 to inform the Intervention Mapping process and lymphedema management program recommendations.

\section{Recommendations}

The findings from this study enhanced the conceptual understanding of the complexity of life experiences among women living in filariasis-endemic areas who 
have lymphedema of the leg. The experiences that women describe suggest that there is not only a spectrum of consequences related to lymphedema itself but also a potential spectrum of coping mechanisms and strategies.

Findings from this qualitative research suggest the need for quantitative studies among women with lymphedema to better understand the distribution of the problems expressed by women, the generalizability of the current study findings, and variation of issues that were noted specifically as related to age and stage of lymphedema. If such research, confirmed the findings in this study, and further, suggested a substantial prevalence of the problems we've identified then the development, implementation, and evaluation of appropriate behavioral interventions as recommended below would be warranted.

\subsection{Health-seeking and self-care}

Training both indigenous healers, considered as lay health advisors by Dominican women, along with physicians in simple, low-cost self-care regimes and issues related to psychosocial distress could increase the early detection, diagnosis, and treatment of lymphedema among women in filariasis-endemic areas. Familyoriented interventions and support groups for women and their families are viable ways to provide psycho-educational opportunities with skill building sessions, reinforcement for self-management efforts and emotional support for women. The often indiscriminate use of injectable antibiotics without medical supervision suggests a need for health education messages about this topic for women during lymphedema management interventions.

\subsection{Psychological distress}

We recommend that the recognition, identification, and treatment of mental, emotional, and psychological distress among Dominican women with lymphedema of the leg, an important and unmet need, be addressed by primary care, dermatology, and mental health programs. Diagnostic tests such as those for depression and quality of life tests for dermatological conditions, illness intrusion, and chronic illnesses could be used to augment overall program efforts to identify and treat psychological distress.

\subsection{Disrupted social connectedness}

We recommend that morbidity control programs consider interventions with specific objectives to help Dominican women enhance or maintain existing social connections and natural social networks, and create replacements for impoverished or extinguished social connections and networks.

\subsection{Stigma}

We recommend that lymphedema management programs include community awareness and public education programs for perpetrators of enacted stigma and specific programs for mediating the negative effects of stigma, whether experienced, perceived, or internalized among women in Ghana and the Dominican Republic. 


\subsection{Program planning}

We recommend that the development of lymphedema management programs be guided by evidence and theory using an Intervention Mapping program planning process. This allows for the efficacious utilization of limited funding and resources and the opportunity to evaluate the effectiveness of the program efforts.

It is the hope of the author that this qualitative study will offer insight into the experiences of women living with lymphedema of the leg; and that findings from this study and further research will assist in determining the best interventions for improving quality of life for all women with this debilitating condition. 


\section{References}

1. Rajan TV. Lymphatic filariasis: a historical perspective. In: Nutman TB, ed. Lymphatic filariasis. Singapore: Imperial College Press; 2000:1-5.

2. Addiss DG, Brady MA. Morbidity management in the Global Programme to Eliminate Lymphatic Filariasis: a review of the scientific literature. Filaria J. 2007;6:2.

3. Molyneux $\mathrm{DH}$, Zagaria $\mathrm{N}$. Lymphatic filariasis elimination: progress in global programme development. Ann Trop Med Parasitol. 2002;96(Suppl 2):S15-40.

4. Ottesen EA, Duke BOL, Karam M, Behbehani K. Strategies and tools for the control/ elimination of lymphatic filariasis. Bull World Health Organ. 1997;75:491-503.

5. Centers for Disease Control and Prevention. Lymphatic filariasis in the Americas: an epidemiologic history. Atlanta, GA: US Department of Health and Human Services; 2002.

6. Gyapong JO, Adjei S, Sackey SO. Descriptive epidemiology of lymphatic filariasis in Ghana. Trans R Soc Trop Med Hyg. 1996;90:26-30.

7. Weller PF, Ottesen EA, Heck L, Tere T, Neva FA. Endemic filariasis on a Pacific island. I. Clinical, epidemiologic, and parasitologic aspects. Am J Trop Med Hyg. 1982;31(5):94252.

8. Ngwira BMM, Tambala P, Perez AM, Bowie C, Molyneux DH. The geographical distribution of lymphatic filariasis infection in Malawi. Filaria J. 2007;6:12.

9. Onapa AW, Simonsen PE, Baehr I, Pedersen EM. Rapid assessment of the geographical distribution of lymphatic filariasis in Uganda, by screening of schoolchildren for circulating filarial antigens. Ann Trop Med Parasitol. 2005;99:141-53.

10. Addiss D, Dimock KA, Eberhard ML, Lammie PJ. Clinical, parasitological, and immunologic observations of patients with hydrocele and elephantiasis in an area with endemic lymphatic filariasis. J Infect Dis. 1995;171:755-8.

11. Noroes J, Addiss D, Agnaldo C, Figueredo-Silva J, Lima G, Dreyer G. Filarial hydrocele: Risk associated with intrascrotal nodules caused by death of adult Wuchereria bancrofti. Trans R Soc Trop Med Hyg. 2003;97:561-6.

12. Ahorlu CK, Dunyo SK, Asamoah G, Simonsen PE. Consequences of hydrocele and the benefits of hydrocelectomy: A qualitative study in lymphatic filariasis endemic communities on the coast of Ghana. Acta Tropica. 2001; 80:215-21.

13. Babu BV, Harza RK, Chhotray GP, Satyanarayana K. Knowledge and beliefs about elephantiasis and hydrocele of lymphatic filariasis and some socio-economic determinants in an endemic community of Eastern India. Public Health. 2004;118:1217.

12. Gyapong JO, Evans D, Aikins M, Gyapong M, Adjei S. The economic burden of lymphatic filariasis in northern Ghana. Ann Trop Med Parasitol. 1996;90:39-48.

13. Babu BV, Swain BK, Rath K. Impact of chronic lymphatic filariasis on quantity and quality of productive work among weavers in an endemic village from India. Trop Med Int Health. 2006;11:712-7.

14. Babu BV, Nayak AN, Dahl K, Acharya AS, Jangrid PK, Mallick G. The economic loss due to treatment costs and work loss to individuals with chronic lymphatic filariasis in rural communities of Orissa, India. Acta Trop. 2002;82:31-8.

15. Gyapong M, Gyapong JO, Adjei S, Vlassoff C, Weiss M. Filariasis in Northern Ghana: Some cultural beliefs and practices and their implications for disease control. Soc Sci Med. 1996;43:235-42.

16. Bandyopadhyay L. Lymphatic filariasis and the women of India. Soc Sci Med. 1996;42: 1401-10.

17. Coreil J, Mayard G, Louis-Charles J, Addiss D. Filarial elephantiasis among Haitian women: social context and behavioural factors in treatment. Trop Med Int Health. 1998; $3: 467-73$.

18. Kanda K. The quality of life among lymphedema patients due to lymphatic filariasis in three rural towns in Haiti. Tampa, FL: University of South Florida; 2004. 
19. Coreil J, Mayard G, Addiss D. Support groups for women with lymphatic filariasis in Haiti. Geneva: World Health Organization/TDR; 2003.

20. Person B, Addiss D, Kay Bartholomew LK, Meijer C, Pou V, Gonzálvez G, et al. "Can it be that God does not remember me?": a qualitative study on the psychological distress, suffering, and coping of Dominican women with chronic filarial lymphedema and elephantiasis of the leg. Healthcare for Women International. 2008;29:349-65.

22. Person B, Addiss DG, Bartholomew LK, Meijer C, Pou V, Borne van den B. Healthseeking behaviors and self-care practices of Dominican women with lymphedema of the leg: implications for lymphedema management programs. Filaria J. 2006;5(1):13.

23. Person B, Addiss D, Bartholomew LK, Meijer C, Pou V, Gonzálvez G, et al. A qualitative study of the psychosocial and health consequences associated with lymphedema among women in the Dominican Republic. Acta Tropica. 2007;103(2):90-7.

24. Person B, Bartholomew LK, Addiss D, Borne van den B. Disrupted social connectedness among Dominican women with chronic filarial lymphedema. Patient Educ Couns. 2007; 68(3):279-86.

25. Person B, Bartholomew LK, Gyapong $M$, Addiss D, Borne van den B. Health-related stigma among women with lymphatic filariasis from the Dominican Republic and Ghana. Soc Sci Med. 2008. In press.

26. Molyneux DH, Hotez PJ, Fenwick A. "Rapid-impact interventions": how a policy of integrated control for Africa's neglected tropical diseases could benefit the poor. PLoS Med. 2005:e336.

27. US Department of Health and Human Services. Life cycle of Wuchereria bancrofti. 2007. Available at: http://www.dpd.cdc.gov/dpdx/html/imagelibrary/filariasis_il.htm. Accessed July 18, 2007.

28. World Health Organization. Lymphatic filariasis: diagnosis and pathogenesis. Bull World Health Organ. 1993;71:135-41.

29. Emory University Lymphatic Filariasis Support Center. Lymphatic filariasis in the Americas. 2005. Available at: http://www.sph.emory.edu/lfsc/americas/americas.html. Accessed December 2, 2006.

30. Michael E, Bundy DAP, Grenfell BT. Re-assessing the global prevalence and distribution of lymphatic filariasis. Parasitology. 1996;112:409-28.

31. World Health Organization. Bridging the gaps. Report of the director-general. Geneva: The Organization; 1995.

32. Durrheim D, Wynd S, Liese B, Gyapong J. Lymphatic filariasis endemicity-an indicator of poverty? [editorial]. Trop Med Int Health. 2004;9:843-5.

33. World Health Organization. Resolution of the Executive Board of the WHO: elimination of lymphatic filariasis as a public health problem. 5oth World Health Assembly. Geneva: the Organization; 1997.

34. Seim AR DG, Addiss DG. Controlling morbidity and interrupting transmission: twin pillars of lymphatic filariasis elimination. Revista da Sociedade Brasileira de Medicina Tropical. 1999;32:325-8.

35. World Health Organization. Prospect for elimination: chagas disease, leprosy, lymphatic filariasis, onchocerciasis. Geneva: the Organization; 1997.

36. Ottesen EA. The Global Programme to Eliminate Lymphatic Filariasis. Trop Med Int Health. 2000;5:591-4.

37. Houston R, Lapointe M, Lammie PJ, Freeman A, Addiss D. The role of salt in global elimination of lymphatic filariasis. Filaria J. 2003.

38. Freeman AR, Lammie PJ, Houston R, LaPointe MD, Streit TG, Jooste PL, et al. A community-based trial for the control of lymphatic filariasis and iodine deficiency using salt fortified with diethylcarbamazine and iodine. Am J Trop Med Hyg. 2001;65:865-71.

39. Beach MJ, Streit TG, Houston R, May WA, Addiss DG, Lammie PJ. Short report: documentation of iodine deficiency in Haitian schoolchildren: implication for lymphatic filariasis elimination in Haiti. Am J Trop Med Hyg. 2001;64:56-7. 
40. Houston R. Salt fortified with diethylcarbamazine (DEC) as an effective intervention for lymphatic filariasis, with lesson learned from salt iodization programmes. Parasitology. 2000;121S:S161-S73.

41. Shenoy RK, Varghese J, Kuttikkal VV, Kumaraswami V. The efficacy, tolerability and safety of diethylcarbamazine-fortified salt in the treatment of the microfilaraemias of brugian filariasis: an open, hospital-based study. Ann Trop Med Parasitol. 1998;92:28593.

42. Dreyer G, Addiss D, Dreyer P, Noroes J. Basic lymphoedema management: treatment and prevention of problems associated with lymphatic filariasis. Hollis, NH: Hollis Publishing Company; 2002.

43. Addiss DM, Mackenzie C. LF disease-clinical management. Am J Trop Med Hyg 2004:71(5 Suppl):12-15.44.

44. Addiss DG, Dreyer, G. Treatment of lymphatic filariasis. In: Nutman TB, ed. Lymphatic filariasis. London: Imperial College Press; 2000. p. 151-99.

45. Babu BV, Nayak AN. Footcare among lymphoedema patients attending a filariasis clinic in South India: a study of knowledge and practice. Ann Trop Med Parasitol. 2003;97: 21-4.

46. Nanda B, Ramaiah KD. Lymphoedema-management measures practiced by cases of chronic lymphatic filariasis. Ann Trop Med Parasitol. 2003;97:427-31.

47. McPherson T, Fay MP, Singh S, Penzer R, Hay R. Health workers agreement in clinical description of filarial lymphedema. Am. J. Trop. Med. Hyg. 2006;74(3):500-4.

48. Rajan TV, Gundlapalli AV. Lymphatic filariasis. Chem Immunol. 1997;66:125-58.

49. Dreyer G, Medeiros Z, Netto MJ, Leal NC, de Castro LG, Piessens WF. Acute attacks in the extremities of persons living in an area endemic for bancroftian filariasis: differentiation of two syndromes. Trans Roy Soc Trop Med Hyg. 1999;93:413-7.

50. Pani SP, Yuvaraj J, Vanamail P, et al. Episodic adenolymphangitis and lymphoedema in patients with bancroftian filariasis. Trans Roy Soc Trop Med Hyg. 1995;89:72-4.

51. Addiss DG, Louis-Charles J., Wendt JM. Epidemiology of "acute attacks" among patients in a treatment program for filariasis-associated lymphedema of the leg, Leogane, Haiti. Abstract 415. Am J Trop Med Hyg. 1999;61(Suppl 3):320.

52. Acton HW, Rao SS. The importance of secondary infections in causation of filarial lymphangitis. Indian Medical Gazette. 1929;64:421-3.

53. Dreyer G, Nores J, Figueredo-Silva J, Piessens WF. Pathogens of lymphatic disease in the Bancroftian filariasis: a clinical perspective. Parasitology Today. 2000;16(12):544-8.

54. Dreyer G. New insights into the natural history and pathology of bancroftian filariasis: Implications for clinical management and filariasis control programmes. Trans Roy Soc Trop Med Hyg. 2000;94:594-6.

55. Lammie PJ, Cuenco KT, Punkosdy GA. The pathogenesis of filarial lymphedema: is it the worm or is it the host? Ann NY Acad Sci. 2002;979:131-42.

56. Ananthakrishnan S, Das LK. Entry lesions in bancroftian filarial lymphoedema patients-a clinical observation. Acta Tropica. 2004;90:215-8.

57. Alexander N, Perry R, Dimber Z, Hyun P, Alpers M, Kazura J. Acute disease episodes in a Wuchereria bancrofti-endemic area of Papua New Guinea. Am J Trop Med Hyg. 1999;61:319-24.

58. Dunyo SK, Nkrumah FK, Ahorlu CK, Simonsen PE. Exfoliative skin manifestations in acute lymphatic filariasis. Trans R Soc Trop Med Hyg. 1998;92:539-40.

59. Kumaraswami V. The clinical manifestations of lymphatic filariasis. In: Nutman TB, ed. Lymphatic filariasis. London: Imperial College Press; 2000. p. 103-25.

6o. Dreyer G, Addiss D, Bettinger J, Dreyer P, Noroes J, Rio F. Lymphoedema staff manual: treatment and prevention of problems associated with lymphatic filariasis. Geneva: WHO/CDS/CPE/CEE/2001.26a; 2001.

61. Weil GJ, Lammie PJ, Weiss N. The ICT filariasis test: a rapid format antigen test for diagnosis of bancroftian filariasis. Parasitology Today. 1997;13:401-4.

62. Serivce MW. Mosquitoes. In: Lane RP, Cosskey RW, eds. Medical insects and arachnids. London: Chapman and Hall; 1993. p. 120-240. 
63. World Health Organization. Global Program to Eliminate Lymphatic Filariasis. WHO weekly epidemiological record. 2006;22:221-32.

64. Pan American Health Organization. Lymphatic filariasis elimination in the Americas. 2002.

65. The Global Alliance to Eliminate Lymphatic Filariasis. The socio-economic impact of LF and the program to eliminate it. 2005. Available at: http://www.taskforce.org/lfsc/ toolkit/overview/seburden.pdf. Accessed April 5, 2006.

66. Gyapong JO, Dollimore N, Binka FN, Ross DA. Lay reporting of elephantiasis of the leg in northern Ghana. Trans R Soc Trop Med Hyg. 1995;89:616-8.

67. Emory University Lymphatic Filariasis Support Center. Lymphatic filariasis elimination in the Dominican Republic. 2007. Available at: http://www.taskforce.org/LFSC/ americas/dominican.html. Accessed July 18, 2007.

68. Gyapong JO. The relationship between infection and disease in Wuchereria bancrofti infection in Ghana. Trans Roy Soc Trop Med Hyg. 1998;92:390-2.

69. Vincent AL, Gonzalvo A, Cowell BC, Nayar JK, Uribe L. A survey of bancroftian filariasis in the Dominican Republic. J Parasitol. 1987;73:839-40.

70 Vincent AL, Rojas UAC, Ayoub EM, Ottesen EA, Harden EG. Filariasis and erisipela in Santo Domingo. J Parasitol. 1998;84:557-61.

71. Patton M. Qualitative interviewing. In: Qualitative research and evaluation methods, 3rd ed. Thousand Oaks, CA: Sage Publications; 2002. p. 339-447.

72. Muhr T. ATLAS-ti version 4.2 [computer software]. Berlin: Scientific Software; 1997.

73. Strauss Al, Corbin J. Basics of qualitative research and evaluation methods. Thousand Oaks, CA: Sage Publications; 1998.

74. Charmaz K. Constructing grounded theory. London: Sage Publications; 2006.

75. Porter S. Validity, trustworthiness and rigour: reasserting realism in qualitative research. J Adv Nurs. 2007;60(1):108-9.

76. Johnson RB. Examining the validity structure of qualitative research. Education. 1997;118(2):282-92.

77. Lincoln YS, Guba EG. Naturalistic inquiry. Newbury Park, CA: Sage Publications; 1985.

78. Le Compte $M$, Preissle J. Ethnography and qualitative design in educational research. London: Academic Press Inc.; 1993. 


\title{
Chapter 2
}

\section{A Qualitative Study of the Psychosocial and Health Consequences Associated With Lymphedema among Women in the Dominican Republic}

\author{
Bobbie Person, David Addiss, L. Kay Bartholomew, Cecilia Meijer, \\ Victor Pou, Guillermo Gonzálvez, and Bart van den Borne
}

Manuscript published as: A qualitative study of the psychosocial and health consequences associated with lymphedema among women in the Dominican Republic. Acta Tropica. $2007 ; 103(2): 90-7$. 


\begin{abstract}
To date, few studies have addressed the sequelae of lymphatic filariasis, a mosquito-transmitted disease, on the quality of life of affected women in the Americas. In this qualitative study, we conducted 28 semi-structured interviews and 3 focus groups of women with lymphedema or elephantiasis of the leg living in filariasis-endemic areas of the Dominican Republic. Women in our study described a spectrum of consequences associated with their lymphedema but physical, functional, and psychological limitations were not always associated with severity of lymphedema. Data suggests that management frameworks need to be expanded to address women's explanatory models of illness, the cultural practices of seeking traditional healers for initial care, psychological distress, coping strategies unique to women, and the practice of self-treating with antibiotics without medical supervision. Further research to better understand the depth and breadth of psychological states and coping strategies of women; health-seeking and selfmanagement practices; and strain on social support networks of women is needed in order to assist health program planners in establishing culturally-tailored and gender-specific interventions for Dominican women.
\end{abstract}




\section{Introduction}

Lymphatic filariasis, a mosquito-transmitted parasitic disease, is estimated to affect more than 2 million people in the Americas with more than 8 million people at risk for infection (1). In the four major filariasis-endemic countries in the region, Haiti, the Dominican Republic, Guyana and Brazil, an estimated 200,000 men have urogenital filariasis, principally hydrocele, and another 100,000 persons, mostly women, suffer from lymphedema of the leg, or its most severe form, elephantiasis $(1,2)$. Lymphatic filariasis is caused by thread-like roundworms that damage the lymphatic vessels and this damage predisposes the person to lymphatic dysfunction and secondary bacterial dermatolymphangioadenitis characterized by fever, chills and painful inflammation and swelling of the limb, sometimes followed by exfoliation of the skin $(3-6)$.

Lymphedema of the leg in filariasis-endemic areas leads to disfigurement of the leg, compromised functional abilities, loss of work and increased economic burdens, diminished social roles and social identity, and psychological distress (713). While some studies focus on the economic and psychosocial impact of lymphedema in filariasis-endemic areas, few have examined in-depth the impact of lymphedema on the lives of affected women in the Americas (14-15).

For this study we used qualitative individual interviewing and focus group discussions (FGD) to obtain an in-depth perspective and understanding of the psychosocial and health consequences associated with lymphedema of the leg among women in the Dominican Republic. We focused on women's current and past experiences, perceptions, beliefs, feelings and expectations for future well-being as it related to having lymphedema.

\section{Materials and methods}

This qualitative study was conducted in the filariasis-endemic country of the Dominican Republic, which shares the island of Hispaniola with Haiti (16). Public health researchers from the Centro Para el Control de Enfermedades Tropicales (CENCET) have identified 10 municipalities in the southern coastal region of the country that are filariasis-endemic areas (17). These areas include densely populated urban neighborhoods or barrios of the cities as well as the poorer communities or bateys associated with rural sugarcane production (18).

Qualitative interviews and FGDs, used to gather data for this study, have been shown to be especially relevant in areas where there has been little previous research and there is a need for discovery, descriptive accounts and an understanding of how people interpret and explain a social phenomenon or personal experience $(19,20)$. Pretested, open-ended guides were constructed to encourage women to discuss a variety of experiences during interviews and FGDs. The guides were translated from English into Spanish by a native Spanish speaker and back-translated into English to assess comparability to the original text.

Purposive sampling was used to recruit women into the study who lived throughout filariasis-endemic areas; had multiple stages of lymphedema of the leg, stages $3-7$, based on the staging criteria of Dreyer et al.; were of diverse ages and occupations; did not engage in, were inconsistent with or who had just begun 
lymphedema self-management activities; and were willing to be interviewed, audiotaped and photographed (21,22).

Using data from a recent epidemiological study on the prevalence of lymphatic filariasis in the Southwestern region of the country, public health officials referred women with observable lymphedema to the Instituto Dermatológico de Cirugia y Piel (IDCP) in the coastal towns of Santo Domingo and Barahona (17). Dermatologists referred approximately 70 women for interviews of which 30 met the screening criteria. One woman declined to be interviewed and one interview tape was damaged leaving 28 women who completed individual interviews. As the study progressed, we recruited 28 additional women into FGDs who met the same study criteria above along with some women practicing consistent lymphedema management activities. Any woman coming into the clinic during the 2-week period prior to each of our visits was invited to participate in a FGD and the first eight women who met the study criteria and agreed to participate were accepted.

A Spanish-speaking Dominican social scientist, and the primary author, assisted by a Dominican translator, conducted interviews and FGDs. The interviews averaged 2 to 3 hours and the FGDs approximately 2 hours. Individual interviews took place in homes, community settings or the dermatology clinic before a woman's first clinical visit and FGDs were conducted at the IDCP in Santo Domingo and Barahona.

Data collected in Spanish were audiotaped, transcribed verbatim, translated into English by a bilingual research assistant and entered as a Word document into ATLAS-ti to facilitate data coding, text searching and analysis (23). Data were then systematically examined for emerging themes and patterns related to the experiences of living with lymphedema. To ensure inter-coder agreement, two coders derived open coding schemes independently on a subset of interviews, then made comparisons to achieve consensus on the coding strategy and repeated the coding independently on another subset of interviews to ensure consistency of coding strategy implementation. Axial coding allowed for the discovery of relational data and by exploring each interview and focus group transcript and developing thematic tables at subsequent readings, we were able to identify the most salient analysis topics and themes the women discussed (24).

\section{Results}

\subsection{Characteristics of women in the sample}

Background characteristics of the 56 women who agreed to participate in our study are shown in Table 2.1. Only minimal individual data were collected on women in the FGDs but they appeared to be of similar demographics as women who agreed to individual interviews. Most women were married or in a free union relationship and literate. More than two-thirds had previously worked at incomegenerating occupations, and although more than half were still working outside the home, most felt they were economically poor. Many women had relatives who had lymphedema. More than two- thirds of all women felt that their condition contributed to emotional problems in their lives and expressed embarrassment over their condition when in public. 
Table 2.1. Background characteristics of women in the study

\begin{tabular}{lc}
\hline \multicolumn{1}{c}{ Background characteristics of only women who were interviewed (N = 28) } \\
\hline Median age & 50.5 years \\
Literate, yes & $22(79 \%)$ \\
Stage 3 lymphedema (moderate) & $10(36 \%)$ \\
Stage 4-6 lymphedema (severe) & $18(64 \%)$ \\
Married or with a partner & $20(71 \%)$ \\
Widowed & $5(18 \%)$ \\
Divorced or single & $3(11 \%)$ \\
Family member with LF & $19(68 \%)$ \\
\hline \multicolumn{2}{c}{ Background characteristics of all women in the study (N=56) } \\
\hline Lymphedema both legs & $22(39 \%)$ \\
Lymphedema left leg & $20(36 \%)$ \\
Lymphedema right leg & $14(25 \%)$ \\
Age <40 years old & $18(32 \%)$ \\
Age >40 years old & $38(68 \%)$ \\
Lymphedema stage3 & $20(36 \%)$ \\
Lymphedema stage 4-6 & $36(64 \%)$ \\
\hline
\end{tabular}

\subsection{Knowledge of cause and transmission of lymphatic filariasis}

The etiology of lymphatic filariasis was unknown to most of the women in our study. All women had personal stories and causal explanations related to their first episode of swelling. Women attributed their lymphedema to spider bites, heredity, pregnancy, witchcraft, circulation problems, standing too long at a disagreeable job, stepping in a variety of magical substances and accidents. Only five women attributed mosquitoes as a possible cause of their lymphedema.

\subsection{Local terms associated with disease}

As in other countries where filariasis is endemic, local terms were used for lymphedema of the leg and episodic bouts of bacterial dermatolymphangioadenitis. The most common terms used to describe lymphedema of the leg were erisipela or dicipela, but occasionally it was described as "a milk leg." An acute episode of bacterial dermatolymphangioadenitis was commonly referred to as a crisis. The term seca, used interchangeably to refer to either a swollen gland in the groin or a large boil that oozes yellow fluid, and masamorra, used to describe maceration or fungal infections between the toes, were both associated with the onset of a crisis.

\subsection{Characterizations of acute bacterial dermatolymphangioadenitis}

Women characterized episodes of acute bacterial dermatolymphangioadenitis, or crisis, quite differently from chronic lymphedema. A 31-year-old woman with severe lymphedema from a FGD, describing a crisis, said, "I have fever so bad. I have to go to bed. I can't even eat. I lose the will for everything. When I don't have a crisis I manage the best I can." Women described a crisis as when the leg swells and becomes very painful, accompanied by high fevers, severe chills, headache, nausea and total incapacitation on average of 2 to 14 days. Women with more severe lymphedema attributed the onset of a crisis to cutting or abrading the skin or getting it wet, particularly if it remained wet between the toes and now avoided those 
situations. Women with less severe lymphedema were more likely to describe a crisis as a random occurrence having vague or supernatural causes. The behavioral dynamics associated with health-seeking behaviors, self-management strategies and treatment regimes also were different for the acute episodes of crisis than for chronic lymphedema.

\subsection{Health-seeking}

All women described exhaustive and expensive attempts at seeking treatment and a cure for their condition by visiting ensalmadores (specialized herbalists), brujos (witchdoctors), curanderos (healers) and trained health providers. One woman with early-stage lymphedema said, "Already, I have been to so many doctors, brujos and healers that I can't remember them all." Most women sought care from indigenous healers first followed by trained healthcare providers. Women were most frequently seeking a "cure" and most felt that any medical intervention that didn't provide a cure was a failure. Three-fourths of the women had sought care at least once during a crisis. For more than half the women, frustration with poor results from treatment provided by indigenous healers and trained healthcare providers resulted in self-management of an acute crisis with injectable antibiotics, topical antibiotics, and oral painkillers without medical consultation.

\subsection{Physical consequences of disease}

Similar to other studies, the most severe physical and functional limitations were associated with crisis episodes of incapacitation and pain regardless of lymphedema stage. Chronic functional impairment affected care-giving functions, income-generating opportunities and social interactions. But, regardless of lymphedema stage, nearly half of the Dominican women continued to care for themselves and their families and maintain income-generating activities. "When $I$ am not in crisis, I have to be at work at 5:30. I have to prepare the food for my husband before leaving. After arriving from work, I rest a little bit, wash the dishes and do everything else," said a woman with severe lymphedema who works as a secretary and takes care of her family.

\subsection{Psychological consequences of disease}

The psychological consequences of embarrassment over the disfigurement of the leg, sadness and depression, loss, loneliness, distress and shame appeared to be a greater burden to women than the physical consequences. Most women said that this condition had caused emotional problems in their lives regardless of lymphedema stage, repeatedly described feelings of great sadness, depression, low self-esteem and feeling socially disconnected. One 50-year-old woman described her emotional distress: "I would spend the whole day crying. I would drink my tears as I cried."

The changing physical appearance of the leg often created a negative body image and challenged positive self-esteem. One 45-year-old woman in a FGD described her grief: "I look at myself in a mirror and my foot looks like this fat, ugly thing. Sometimes I feel so sad and depressed. Who am I now?" The physical disfigurement of the leg also created embarrassment and shame in social 
interactions. Three-fourths of the women identified loss of self-esteem and social identity as major personal losses. A 65-year-old former cook described her attempts at hiding her condition: "I wear long dresses to hide the leg because I feel ashamed to be seen like this. I am not a real person any more. I hide from people now." A few women expressed ultimate despair with their condition and had even thought of killing themselves

Women also described losses associated with aging. One woman with lymphedema for more than 30 years talked about the losses in her social network as she has aged: "I had a good life long ago. Many people I knew have now died. Those were people who helped me. Now I have nothing and no one."

Despite experiences of physical limitation and psychological loss, women overall were hesitant to classify themselves as disabled, and a few women even described positive social comparison with others who had greater physical impairments and limitations. Women most often identified their religious beliefs and faith as providing the strength to live with this condition.

\subsection{Occupational and economic consequences}

Occupational and economic losses, exacerbated by exhaustive health-seeking attempts and treatment costs, created an economic toll among the women. These women had been beauticians, teachers, domestic workers, factory workers, cooks and bakers, housewives and public servants. While some were unable to perform their jobs, almost half the women continued to work, but at lower paying and lower status jobs, in order to contribute economically. One woman, describing her current situation, said, "I worked as a cook in a hospital for 14 years, then I had to quit because of my big leg. Now I make only pennies." We interviewed three women with moderate and severe lymphedema who, surprisingly, continued to not only work in the home or outside the home but were working toward better-paying occupations. " $I$ am a secretary, and when I get my official recognition I'll practice law," said a 52year-old student.

Health-seeking and treatment costs were often characterized in both tangible figures and intangible outcomes. "The youngest child of mine takes me to the capital when I get very ill. Each time he spends more than 6,000 pesos [\$180 USD]. Between the doctors, taking me to the clinic, the hospital, taking me here and there, he spends all his money. Look how poor we are because of this leg," said a 65-yearold woman with advanced lymphedema. Almost all women relied on additional economic support from family members to cope with the burdens of disease.

\subsection{Social consequences of disease}

The impact of lymphedema on the personal and social relationships of affected women varied. Women expressed varying attitudes, beliefs and experiences related to love, marriage and sex. Three women felt that true love allowed one's impairment to be overlooked and that a husband's sense of burden would determine whether he stayed with his wife. Some married women, both with mild and severe lymphedema, described their spouses as supportive and caring despite their condition. A few women even described how they married after lymphedema 
developed. A woman in a traditional marriage said, "I use to think that no one will want to marry me because I have this really ugly leg. But I did marry and everything has been fine. My husband has never talked bad to me and on the contrary he has helped me to face it, to struggle to get back my life." But there were also women who felt that their lymphedema contributed to the stress in their marriage or even to divorce. One 38-year-old woman said, "Yes, this condition has caused a few problems. Our [sexual] relations have been affected quite a bit. He always says there is not another woman, but you know how it is. I think he deceives me." Almost one-third of the married women in good relationships felt that their own situation was unique, that it would be difficult for other women with lymphedema to get married. For a few sexually active women sex was only interrupted when they experienced a crisis. "It affects me when I have the crisis. Then I am in no condition to have sex. The leg grows bigger and it is so painful." One woman's physician recommended that she and her husband cease sexual relations due to the size of her leg. She said, "One day the doctor called my husband and told him to treat me not like a wife but as a sister because my obligations as a married woman were affecting me." Another woman who described having a major marital problem humorously said that it was a result of having a bad husband rather than a bad leg. Overall, women felt there was little discrimination associated with having a family member with lymphedema. Most women felt that their lymphedema would not affect the marriage potential of anyone in their family and had examples of many family members who had married.

Women were provided direct and indirect support most frequently from close family members. The concern of a family member was usually the impetus for a woman's first visit to seek treatment for her condition. Women most often derived emotional support from other female family members. While family members provide emotional and instrumental support, women also told us that their families suffer emotional, psychological and economic burdens due to their lymphedema. One 60-year-old woman said, "Oh, they [my family] have suffered because of my ailment. My children get desperate and so does my husband. My sister said that my disease has cost more than 1 million pesos. She died just wanting to see me better."

A few women described significant experiences of enacted stigma and discrimination by health care providers who asked them to sit somewhere other than the waiting room or who didn't want to touch their leg during examination. While children were more likely to tease or taunt women, adults often were more covert about discrimination with fear of contagion being a source of fear. A woman with severe lymphedema said, "People in my neighborhood are afraid of getting infected by my clothes and won't help me [wash my clothes]. I know because I have heard their conversations." While most women felt that people often pitied them they didn't think that they would necessarily go out of their way to avoid them. Despite having some understanding and supportive experiences with others in their social and community network and even having family and friends who visited when they were housebound, many women still felt socially disconnected and experienced grief over who they used to be and who they could have been. 


\subsection{Future expectations}

Some women were still hopeful for a cure and even optimistic about the future course of their disease even if unrealistic. But other women expressed little hope for a better future. "The future is not for me. You see I have no opportunity. I have no hope," said one woman with severe lymphedema. A few women expressed concern that their lymphedema would be exacerbated by conditions brought on by aging. "In the following years I think my condition will get worse because you know age is very unforgiving. You start to come into your age, getting older and your illness affects you more," said a 66-year-old woman with poor health and a small social network.

\section{Discussion}

The few studies specific to women with lymphedema in Haiti and India indicate that they are severely burdened by the physical and social consequences of disease $(14,15)$. Dominican women described a spectrum of consequences associated with their lymphedema and noted that quality of life was dynamic depending upon changes in their health status and circumstances over time. Similar to participants in studies in other endemic areas of the world, the women in our study had local terms for lymphedema and acute bacterial dermatolymphangioadenitis, and few women knew the etiology of lymphatic filariasis and its association with their own lymphedema $(14,25)$. Most of the women derived their own explanatory model of illness, whereby they appraised and interpreted their symptoms within the experiences and contextual factors of their culture and daily lives (26). Women most often attributed their lymphedema to spider bites, heredity, pregnancy, witchcraft, circulation problems and accidents, plausible within their cultural paradigm, these explanatory models failed to facilitate early treatment seeking to slow the progression of lymphedema. Similar to other studies conducted in a Latin culture, women described an initial problem-solving phase in their explanatory model of illness where family and friends acted as lay health advisers providing health referrals to ensalmadores, curanderos, and brujos $(27,28)$. Ensalmadores were most likely to have specific procedures, incantations and remedies for erisipelas, while other traditional providers offered generic potions, creams, injections and prayers. It was often only the failure of these traditional methods of care that resulted in women seeking care by a trained healthcare provider. Even when seeking care from a trained physician, women were often misdiagnosed and provided only limited relief. A poor understanding of the disease, high healthcare costs and frequent misdiagnosis by physicians led many women to eventually self-administer a regime of antibiotic injections and oral painkillers without medical consultations. Exhaustive attempts at seeking health treatments and a cure often reinforced their beliefs that nothing could be done for their condition despite their continued hopes for a cure in the future.

Physical and functional limitations were not always associated with severity of symptoms or, as described in this study, stage of lymphedema. Some women continued to be caregivers, hold jobs, and remain involved in social activities despite advanced-stage lymphedema, while others with less severe disease were unable to do so. Despite functional limitations and psychological losses, many women were 
hesitant to consider themselves disabled. In contrast to the findings of studies in Ghana and India but similar to the results of some studies in Haiti, we found that many women with lymphedema in the Dominican Republic were married and reportedly had supportive husbands $(14,15,29)$. While several women described situations that resulted in the cessation of sexual relations with their husbands, most women said the disease had not affected sexual activity in their relationships. Support was most often intergenerational, including an array of family members that helped to moderate the distressing consequences of a woman's condition (30). Family members and particularly husbands most often provided instrumental assistance of money, food and transportation despite the considerable burdens placed upon them. Similar to other research on women and stress, women in our study most often turned to other female family members and friends for emotional support (30).

A striking finding was the breadth of psychological distress experienced by women. Embarrassment over the disfigurement of the leg, sadness and depression, loss, loneliness and shame contributed to a ubiquitous state of psychological distress for women across all stages of disease. Repeatedly and with great emotion, women described feelings of sadness and depression with episodes of crying. Issues related to negative body image appeared to be exacerbated as women tried to hide their enlarged, disfigured legs. The economic ramifications of an altered occupational role and the associated loss of social identity often negatively influenced a woman's perception of self-worth. But while the sequelae of disease often resulted in a modification or loss of an occupational role, some women with limited and severe disease continued to care for themselves and their families, maintain incomegenerating activities, and even plan for new occupations. Despite the pervasive nature of psychological distress, women appeared to be coping across multiple domains of their lives. Religious beliefs, a positive outlook and hope provided the basis for positive future expectations while aging, weariness and hopelessness reinforced negative future expectations.

Recent studies on coping suggest that underlying physiological and behavioral differences may affect how women cope with adversity (31). Researchers studying the biobehavioral responses of women to stress now posit that women respond to stress differently than men and characterize this response as "tend-and-befriend." They assert that women have an adaptive response to seek affiliation and social contact with others during times of stress. Findings from our study suggest that further understanding of the depth and breadth of psychological distress experienced by women, as well as the coping strategies they use to moderate distress, will be critical to providing comprehensive lymphedema management programs for women. As seen in other devastating chronic diseases, coping strategies are learned in a cultural context (32). Examining more thoroughly the explanatory models of illness and understanding the health-seeking behaviors of Dominican women could assist health program planners in establishing culturally tailored interventions that seek to identify women soon after onset of lymphedema and provide appropriate treatment and ongoing support. Discerning the role of family and social support, the types of support offered and use of support systems could contribute to the development of methods and strategies to motivate and reinforce regimens of care that reduce the 
chronic effects of morbidity among women. Further research is also necessary to determine the educational and training needs of not only trained physicians but also of indigenous lay health advisers, who appear to be the first source of care for women in the Dominican Republic.

\section{Conclusion}

The findings from this study enhance our conceptual understanding of the complexity of life experiences among women living in filariasis-endemic areas who have lymphedema of the leg. The experiences that women describe suggest that there is not only a spectrum of consequences related to lymphedema itself but also a potential spectrum of coping mechanisms and strategies. A better understanding is needed of the unique needs of women with lymphedema in filariasis-endemic countries and of the coping strategies within the cultural context of their daily lives if lymphedema management programs are to ameliorate the effects of morbidity. Our findings also demonstrated that there is a lack of trained healthcare providers to deal with the needs of those affected by lymphedema. It is essential to determine training mechanisms whereby knowledge and standard of care practices could be taught to formally trained as well as lay health advisers who interact with these women. 


\section{References}

1. US Department of Health and Human Services. Lymphatic filariasis in the Americas: an epidemiologic history. Atlanta, GA: Centers for Disease Control and Prevention; 2002.

2. Vincent AL, Gonzalvo A, Cowell BC, Nayar JK, Uribe, L. Research notes: a survey of bancroftian filariasis in the Dominican Republic. J Parasitol. 1987;73:839-40.

3. Connor DH, Palmieri JR, Gibson DW. Pathogenesis of lymphatic filariasis in man. Parasitol Res. 1986;72:13-28.

4. Pani S, Yuvaraj J, Vanamail P, Dhanda V, Michael E, Grenfell BT, et al. 1995. Episodic adenolymphangitis and lymphedema in patients with bancroftian filariasis. Trans R Soc Trop Med Hyg. 1995;89:72-4.

5. World Health Organization. Expert Committee: lymphatic filariasis: diagnosis and pathogenesis. Bull World Health Organ. 1993;71:135-41.

6. Partono F. The spectrum of disease in lymphatic filariasis. In: Filariasis: Ciba Foundation Symposium 127. Chichester, UK: John Wiley \& Sons; 1987:15-31.

7. Babu BV, Harza RK, Chhotray GP, Satyanarayana $\mathrm{K}$. Knowledge and beliefs about elephantiasis and hydrocele of lymphatic filariasis and some socio-economic determinants in an endemic community of Eastern India. Public Health. 2004;118:121-7.

8. Gyapong JO, Evans D, Aikins M, Gyapong M, Adjei, S. The economic burden of lymphatic filariasis in northern Ghana. Ann Trop Med Parasitol. 1996;90:39-48.

9. Kanda $\mathrm{K}$. The quality of life among lymphoedema patients due to lymphatic filariasis in three rural towns in Haiti. Tampa, FL: University of South Florida; 2004.

10. Kumari AK, Harichandrakumar KT, Das LK, Krishnamoorthy K. Physical and psychosocial burden due to lymphatic filariasis as perceived by patients and medical experts. Trop Med Int Health. 2005;10:567-73.

11. McPherson T. Impact on the quality of life of lymphoedema patients following introduction of a hygiene and skin care regimen in a Guyanese community endemic for lymphatic filariasis: a preliminary clinical intervention study. Filaria J. 2003;2:1.

12. Person B, Addiss DG, Bartholomew LK, Meijer C, Pou V, van den Borne B. Healthseeking behaviors and self-care practices of Dominican women with lymphoedema of the leg: implications for lymphoedema management programs. Filaria J. 2006;5:13.

13. Ramaiah KD, Guyatt H, Ramu K, Vanamail P, Pani SP, Das PK. Treatment costs and loss of work time to individuals with chronic lymphatic filariasis in rural communities in south India. Trop Med Int Health. 1999;4:19-25.

14. Coreil J, Mayard G, Louis-Charles J, Addis D. Filarial elephantiasis among Haitian women: social context and behavioral factors in treatment. Trop Med Int Health. 1998;3:467-73.

15. Bandyopadhyay L. Lymphatic filariasis and the women of India. Soc Sci Med. 1996;42:1401-10.

16. Central Intelligence Agency. Dominican Republic. The World Factbook. 29 March 2006. Available at: http://www.cia.gov/cia/publications/factbook/geos/dr.html. Accessed April 5, 2006.

17. Emory University Lymphatic Filariasis Support Center. Lymphatic filariasis elimination in the Dominican Republic. Available at: http://www.taskforce.org/LFSC/americas/ dominican.html. Accessed April 5, 2006.

18. Huang S, Paulino F, Gonzálvez G, Dietz V, Stroh G, Addiss D. Elimination of lymphatic filariasis in the Americas: rapid assessment in the Dominican Republic to determine the need for an elimination program. In: The 47th Annual Meeting of the American Society of Tropical Medicine and Hygiene, San Juan, Puerto Rico. Am J Trop Med Hyg. 1998;Suppl:245.

19. Patton MQ. Qualitative interviewing. In: Qualitative research and evaluation methods, 3rd ed. Thousand Oaks, CA: Sage Publications; 2003:339-427. 
20. Berg B. Qualitative research methods for the social sciences. $5^{\text {th }}$ ed. London: Allyn and Bacon; 2003.

21. Charmez K. Constructing grounded theory: a practical guide through qualitative analysis. London: Sage Publications; 2006.

22. Dreyer G, Addiss D, Dreyer P, Noroes J. Basic lymphedema management: treatment and prevention of problems associated with lymphatic filariasis. Hollis, NH: Hollis; 2002.

23. Muhr T. ATLAS-ti version 4.2 [computer software]. Berlin: Scientific Software; 1997.

24. Strauss A, Corbin J. Basics of qualitative research: techniques and procedures for developing grounded theory. Thousand Oaks, CA: Sage Publications; 1998.

25. Gyapong M, Gyapong JO, Adjei S, Vlassoff C, Weiss M. Filariasis in northern Ghana: some cultural beliefs and practices and their implications for disease control. Soc Sci Med. 1996;43:235-42.

26. Leventhal H, Brisseette I, Leventhal E. The common-sense model of self-regulation of health and illness. In: Cameron $\mathrm{L}$, Leventhal $\mathrm{H}$, editors. The self regulation of health and illness behavior. London: Routledge; 2003:42-65.

27. López-De Fede A, Haeussler-Fiore D. A report: an introduction to the culture of the Dominican Republic for rehabilitation service providers. Center for International Rehabilitation Research Information and Exchange. 2002. Available at: http:// www.cirrie.buffalo.edu/domrep.html. Accessed April 5, 2006.

28. Finkler $K$. Sacred healing and biomedicine compared. In: Brown $P$, editor. Understanding and applying medical anthropology. New York: McGraw-Hill; 1998:11828.

29. Ahorlu CK, Dunyo SK, Koram KA, Nkrumah FK, Aagaard-Hansen J, Simonsen PE. Lymphatic filariasis related perceptions and practices on the coast of Ghana: implications for prevention and control. Acta Trop. 1999;73:251-61.

30. Taylor SE. Stress. In: Health psychology. $5^{\text {th }}$ ed. New York: McGraw-Hill; 2003:411-29.

31. Taylor SE, Klein LC, Lewis BP, Gruenewald TL, Gurung RAR, Updegraff JA. Biobehavioral responses to stress in females: tend-and-befriend, not fight-or-flight. Psychol Rev. 2000;107:411-29.

32. Waxler NE. Learning to be a leper: a case study in the social construction of illness. In: Brown P, editor. Understanding and applying medical anthropology. New York: McGraw-Hill; 1998:147-57. 


\title{
Chapter 3
}

\section{"Can it be that God does not remember me": \\ A Qualitative Study on the Psychological Distress, Suffering, and Coping of Dominican Women with Chronic Filarial Lymphedema and Elephantiasis of the Leg}

\author{
Bobbie Person, David Addiss, L. Kay Bartholomew, Cecilia Meijer, Victor Pou, \\ Guillermo Gonzálvez, and Bart van den Borne
}




\begin{abstract}
The psychological states of Dominican women with chronic lymphedema and elephantiasis of the leg and the coping strategies they used to ameliorate the negative psychological effects of this condition were explored using modified precepts of grounded theory method. Qualitative data were gathered through in-depth interviewing and focus group discussions held in the Dominican Republic. Thematic results found that compounding their physical disfigurement, functional limitations, and social losses were feelings of depression, embarrassment, social isolation, and despair. Adaptive problem solving and emotion-focused coping strategies that emerged during analysis are also discussed. It is recommended that management of psychological distress should be a significant component of lymphedema management programs in developing countries.
\end{abstract}




\section{Introduction}

Lymphatic filariasis, a mosquito-transmitted parasitic disease, is estimated to affect more than two million people in the Americas, with more than eight million people at risk for infection (1). In the four major filariasis-endemic countries in the region-Haiti, the Dominican Republic, Guyana, and Brazil-an estimated 200,000 men have urogenital filariasis, principally hydrocele, and another 100,000 persons, mostly women, suffer from lymphedema of the leg, or its most severe form, elephantiasis (1). Of those women with lymphedema or elephantiasis of the leg, many are women in the Dominican Republic on the island of Hispaniola.

Endemic lymphatic filariasis in the Dominican Republic, caused by the parasite Wuchereria bancrofti, damages the lymphatic system and impacts the physical, functional, social, economic, and psychological domains of a patient's life (2-6). While the disease burden of lymphedema of the leg is greater among women than men, little gender-specific in-depth research has been conducted to better understand the specific effects of the condition on women's quality of life (7-9). This lack of research is particularly evident in the psychological and emotional domains of women with lymphedema. Developing countries with few resources are seldom able to assess and strategically plan for the negative psychological states associated with chronic conditions, particularly those resulting from infectious diseases (10). We conducted this qualitative study to understand better the psychological states that accompany the physical and functional limitations of disease, as well as the coping strategies that women use to ameliorate negative psychological states.

\section{Methods}

\subsection{Study site}

The Dominican Republic, sharing the island of Hispaniola with Haiti, is a filariasis-endemic country in the Caribbean Sea (11). This study was conducted in filariasis-endemic areas of the country. Previous research had shown a microfilaremia prevalence of $12.8 \%$ (29/227) among schoolchildren 4 to 19 years old in La Cienaga, a densely populated urban barrio in Santo Domingo, and a microfilaremia prevalence of $4.1 \%(10 / 243)$ in the poorer, rural shanty-towns or bateys of Santa Cruz de Barahona (12). Both of these areas are included in the study.

\subsection{Qualitative design and approach}

Qualitative data were gathered through in-depth interviews, focus group discussions, field notes, and photographs of women in their homes (13-15). A semistructured interview guide served to structure the in-depth interview sessions of 28 women with lymphedema. The general topics in the guide revolved around impact of disease, health-seeking behaviors, and economic vulnerability. During interviews, women discussed many topics, including physical bodily changes, influence of lymphedema on functioning, psychological states, emotional experiences, occupations and loss of work, personal and social relationships, health-seeking behaviors both traditional and western, self-care practices, stigma, coping strategies, 
and loss and grief. A native Spanish speaker translated the questions from English to Spanish and then translated them back into English to ensure congruence with the original intent of the questions in the guide. Questions and probes in the interview guide, pretested on a sample of local women with lymphedema, were modified to correspond with local dialect, cultural meanings, and literacy levels of the women. Three focus group discussions, with a total of 28 women, were also conducted at the Instituto Dermatológico de Cirugia y Piel (IDCP) to validate data, explore theme interpretation, and explore additional ideas and concepts from individual interviews (14). A semi-structured focus group moderator's guide was developed to ensure exploration of specific ideas, concepts, and categories that emerged during interview sessions specific to the role of different types of traditional healers, the types and results of traditional remedies that women had used in the past, the misdiagnosis of their disease by trained physicians, and the role of faith as a coping mechanism for living with their condition.

We used constant comparison derived from modified precepts of grounded theory $(16,17)$. This is a theoretical methodology often used when little is known about a topic. In this inductive method, the field data define the conceptual categories from the point of view of the women being interviewed allowing for the continuing exploration of ideas and themes as they arise during the interview. In a modified version of grounded theory the findings from the analysis of several interviews contributed to new explorations in subsequent interviews. Interviewing was conducted through numerous visits over a two-year period allowing for a review of the interview session at the time of the interview followed by review and preliminary analysis of several interviews prior to the next trip to conduct further interviews. This allowed for data from earlier interviews to inform subsequent interviews. Data saturation was achieved at 24 participants. Using in-depth interview findings to guide focus groups, comparing the interview findings with focus group findings, comparing notes following interviews, and comparing data against existing literature allowed for triangulation of data, a qualitative methodology to validate findings by comparing them to outcomes derived from other methodologies.

\subsection{Recruitment of women}

In 2002, an estimated 63,325 individuals in the Dominican Republic were infected with lymphatic filariasis, with the prevalence of lymphedema of the leg, predominately of women, at $0.7 \%$ (1).We used targeted or purposive sampling to select women with specific clinical stages of lymphedema of the leg to study their life experiences (18). In 2002, the Centro Para el Control de Enfermedades Tropicales (CENCET) conducted a lymphatic filariasis census in the southern, filarial-endemic region of the country prior to the administration of a program of mass drug distribution for the interruption of disease transmission. The data collected by trained health workers identified anyone with observable lymphedema of the leg. Using these data, public health officials referred women to the IDCP in the coastal towns of Santo Domingo and Barahona. Dermatologists in each clinic screened and then referred women who fit the study criteria to be interviewed. Theoretical sampling was used to recruit women into the study who lived throughout filariasisendemic areas; had multiple stages of lymphedema of the leg, stages $1-7$, based on 
the staging criteria of Dreyer et al.; were of diverse ages and occupations; participated in no prescribed lymphedema management activities or were inconsistent with lymphedema management activities; and were willing to be interviewed, audiotaped, and photographed $(19,20)$. Based on the staging criteria of Dreyer, permanent (non-reversing) lymphedema is considered Stage 3 if shallow skin folds are present; Stage 4 if there are protrusions or "knobs"; Stage 5 if deep skin folds are present; Stage 6 if "mossy lesions" are present; and Stage 7 if the patient is unable to perform activities of daily living. Of approximately 70 women, 30 met the screening criteria and were referred to us for in-depth interviews. During initial interviewing, women with stages 1 and 2 lymphedema described the impact of lymphedema on their lives as none or only a minor, temporary discomfort and felt that the avenues of inquiry did not pertain to their situation. When this emerged as a significant finding, we modified future recruitment to include only women with lymphedema of stages $3-7$.

As the study progressed, we recruited women into focus group discussions who met the same study criteria above along with some women practicing a lymphedema management intervention. Focus group participants were recruited to specifically elaborate, validate, or provide further dimensions on findings that had emerged from the interviews. Any woman coming into the clinic during the two-week period prior to each of our visits was invited to participate in a focus group discussion and the first eight women who met the study criteria and agreed to participate were accepted.

\subsection{Procedures}

In keeping with the protocol approved by the US Centers for Disease Control and Prevention (CDC) and CENCET institutional and ethical review boards, we obtained informed consent from every woman who participated in the study. Indepth interviews of 28 women with lymphedema took place in their homes or in the dermatology clinic prior to their first clinical visit. A Dominican social scientist and the primary author, assisted by a translator from the Dominican Republic, conducted the in-depth interviews in Spanish. The interviews averaged two to three hours. Twenty-eight women also participated in three focus group discussions conducted in Spanish. Two focus groups were conducted at IDCP in Santo Domingo, and one was conducted at the IDCP in Barahona. The focus groups were led by a Dominican social scientist with the primary author and a Dominican research assistant as observers and note takers.

\subsection{Data management and analysis}

A native Spanish speaker transcribed each audiotape and then translated it into English as a Microsoft Word document. Transcripts were then entered into ATLAS-ti, a software management package for qualitative data, allowing for systematically searching text, coding data, identifying emerging categories, themes, and analyzing data (21). To ensure inter-rater reliability, two coders derived coding schemes independently on a subset of interviews, then made comparisons to derive consensus on the coding strategy, and repeated the coding independently on another 
subset of interviews. Themes and constructs that emerged from the open coding strategy allowed for axial coding. By developing thematic tables, we were able to categorize the topics, themes, and constructs that women discussed and extract direct quotes for data analysis and determined their relationship to one another $(17,22)$. Consensus among the research team was required in the interpretation and meaning of the chosen texts related to the psychological states and coping strategies. Selective coding matrices allowed for a theoretical framework of psychological distress to be identified which included the following themes: embarrassment and shame, personal, and social losses, social isolation, enacted stigma, and hopelessness and despair. Coping strategies were then explored within this framework.

\section{Results}

Data from 28 of the 30 women we approached were included in the analysis. One woman refused to be interviewed and one damaged interview tape was not included in the data set. Twenty-eight additional women participated in three focus group discussions. Of the 56 women participating in the study, $40 \%$ had lymphedema in both legs, $36 \%$ in just the left leg, and $24 \%$ in just the right leg. The women ranged from 26 to 80 years old. Most women in the study were older, with $67 \% 40$ to 80 years old and $33 \%$ younger than 40 . Two-thirds of the women presented with more severe lymphedema, stages $4-6$.

Of women who just participated in the in-depth interview, $78 \%$ are literate; $46 \%$ are currently heads of household; and $71 \%$ are married, in a free union, or common-law marriage, $18 \%$ widowed, and $11 \%$ single. Seventy-five percent have previously worked at income generating occupations, and although $57 \%$ currently work outside the home, most still consider themselves poor. At least $82 \%$ of the women consider religion important in their lives and are Catholic or Pentecostal. More than $60 \%$ of the women who just participated in the in-depth interview had relatives who had lymphedema.

\subsection{Psychological distress and suffering}

Women of all ages across all stages of lymphedema described a pervasive sense of psychological distress despite their coping mechanisms. Themes of psychological distress that emerged were embarrassment and shame; sadness, depression, and fear; personal and social losses; social isolation; stigma; and hopelessness and despair.

\subsection{Embarrassment and shame}

A common experience across all stages of lymphedema in our study was embarrassment associated with the disfigurement of the leg. Even women with less severe stages of disease described being ashamed of their legs. "Everyone says something about my leg. 'Look at your ugly leg! Is your leg getting somewhat relieved?' I get so embarrassed." Women also described embarrassment related to their inability to wear shoes or specific types of clothing. "I can't go out because I can't put my shoes on. My clothes don't fit, my pants don't fit, and my shoes don't fit. It makes me so depressed!" 
Women described experiences of being teased, as well as feelings of shame associated with embarrassment and fear of discrimination. A few women described teasing from adults, but generally children teased them. "Sometimes the children in the street mock me. Sometimes they say, 'Hey, look, she has a fat leg bigger than the other.' but they don't call me really bad names."

Most women had internalized feelings of shame. "Sometimes I don't walk. I hide my leg. I am ashamed. I have one good leg and the other bad. I can't even look at other people's legs. My leg is so fat." Women particularly tried to hide the condition in social situations. "I feel bad. Some people can look normal when they are sick. But in my case that does not happen. People look at me as if they were looking at something strange, very, very strange, something they have never seen."

\subsection{Sadness, depression, and fear}

All women described being depressed at some point. "Honestly, how am I not going to feel sad? I get very depressed and it goes deep. I am always anxious." Other women expressed specific fears that created emotional distress and depression. "I think that my leg is going to explode or maybe they will cut my leg off. I cry so much. My tears are many. I am so afraid." Many women were depressed during acute episodes of bacterial adenolymphangitis that result in great pain and swelling of the leg. "Well, I get really depressed when I am sick with the leg. When I have a crisis, I think there is no escape from this leg. I buy so many medications and nothing helps."

\subsection{Personal and social losses}

Women expressed loss across multiple domains of their lives, many of which help them define themselves as women in personal and social ways. "I have many emotional losses. My intimate relations with my husband have decreased because I just don't feel well. There are many things that have decreased." Many women grieve a loss of normalcy. "I am sad because I always have to wear pants. I feel so depressed because I can't make my life normal. My life has changed a lot. You feel sick all the time. Doctors don't know what you have. It is not the same to feel like a healthy person as opposed to a sick person. It is such deep sadness that you can't imagine."

Women also expressed sadness over loss of occupational roles. "It is very bad because I am rarely gone to my business anymore. I cannot spend too much time standing up. My legs are so swollen. It is sad for me." Some women even revised their occupational roles by labeling themselves as sick. "I don't dedicate myself to anything because I am a sick person. What am I going to dedicate myself to?" The loss of function often resulted in a loss of independence and contributed to social losses as well. "You cannot just get up and go for a walk by yourself. You have to have help. I am changed now. I don't go on outings anymore. It makes me sad."

\subsection{Social isolation}

While functional loss, embarrassment over the disfigurement of the leg, and lack of appropriate footwear were most often described as reasons contributing to 
social isolation, women also described other situations. One woman described her feelings of social isolation, "My other daughters treat me badly. They don't look at me because of this disease. I feel bad because they don't treat me the way they treated me before. They don't help me. They stay away. I think they are afraid or repulsed. Because of this disease they don't love me." Although other people isolated some women, other women isolated themselves. "I feel sad. I don't go out unless it's really necessary. I am always locked up. I don't like people looking at my legs. I feel very depressed and sad. I get drunk around here sometimes. I am alone, and I don't spend time with anyone any more."

\subsection{Enacted stigma}

A few women described examples of discriminatory experiences know as enacted stigma. "Approximately two months ago, I felt very bad. My self-esteem was on the floor because of a specific person. A person who does my pedicure and manicure suddenly told me she was risking herself when she did my feet because I had a contagious disease. She told me this in front of several people and made me feel so bad and embarrassed." The stigmatizing situations described by women were most frequently associated with fear of contagion. "A nephew of mine called me smelly at a family reunion. And my son said I had a rotten leg and rejected my attentions. He said he was afraid of it. I had to find someone to tell him that it was not contagious, but he didn't believe it." Women more often described examples of perceived and felt stigma, which are often internalized feelings associated with embarrassment and shame.

\subsection{Hopelessness and despair}

For some women their situation was so psychologically distressing that they felt hopeless and described great despair. "Sometimes when I am depressed, I start to think, 'Can it be that God does not remember me?" Some women felt that their entire lives centered on their leg. "I have felt desperate. I feel so sad that it commands my life. I am hopeless. I think that medicine will not find a cure for me." Women even described thoughts of wishing they were dead. "I have thought sometimes that it would be better to be dead because then I can stop suffering."

\subsection{Coping with psychological distress and suffering}

Women described thoughts, perceptions, and behaviors that reflected adaptive coping strategies used to deal with chronic psychological distress and suffering. Some women thought that the cause of their disease was the result of supernatural circumstances. "I thought I had stepped in a magic powder left for someone else. I walked around to 14 witchdoctors, and none of them did anything. Everyone told me the same thing but nobody cured me. Then I decided to leave it up to the Lord because the Lord can fix everything." Women across all stages gained strength to cope from religious faith. "I am a woman who prays a lot. The people who have faith in God and live through prayer don't let themselves be dominated by the depression. Even if it takes over you, one tries to reject it with God's help." Even when teased, women used faith to cope. "When I notice that they want to tease me, I 
take my Bible and start reading it. That's what I do." Some women, accepting of their condition, used faith to cope with their limitations and to provide hope for improvement. "The Lord gives me the power always. I hope he helps me get better and gives me the strength to walk to church again. I ask the Lord for my health." A number of women considered the possibility of a future miracle. "If the Lord wants, he can give a miracle to anyone. All you have to do is have faith."

Some women found solace in comparing their condition with others who had conditions worse than lymphedema. "Every time I go out, I see people with worse conditions than mine; those are the things that comfort me." For some women life was not reduced to just having a bad leg. One woman described her philosophy: "Life doesn't have anything to do with a leg. I saw a girl on television, a girl who was born without arms and only one leg, and she studies computers and dance. Do you think that I would like to commit suicide because of my leg? Impossible! I have my legs and walk wherever I want! "

Many women in this study drew on the emotional support of family and friends. "My husband comforts me, my neighbors give me advice, people come to pray and they give me advice. Always they are there for me." A few women identified their affiliation with close neighbors and friends in preventing social isolation and assisting in problem solving. "Thank God I don't get isolated by the people I work with. On the contrary they try to help me find solutions for my problems. They tell me, 'Go to the doctor,' they always have me on their minds."

Some women described caring for others as a coping strategy. "I have this little glass case with little treats and juice in it so I can earn some money. I have always wanted to take care of my children and because of that, I try to work despite this condition." Women even described assertively managing situations that were particularly hurtful. "The other day someone told me that in order for me not to suffer anymore I should cut my leg off. I told him that was stupid and hung up the phone on him!"

A few women even used humor to cope. "When people laugh at me, I cooperate with them because if I don't they'll continue making fun of me. So if they ask, 'Why is your leg that fat?' I say it's because it ate lot and is now very full. Then they move on."

Women also expressed hope for new information and treatments. "I had been so depressed because I hoped for a cure. Now I am feeling better because the doctor gave me information about this condition. Before, I did not know anything. I hope for more." Occasionally a woman would talk about the need to support other women with this condition. "We cannot lose hope; we should be lifting each other's spirits. I am not ashamed of anything and of course, people will always talk. But we cannot pay attention to them because then you get sad and you feel more ashamed. Those of us with this problem, we should help each other." 


\section{Discussion}

\subsection{Psychological distress}

Similar to studies in Haiti, India, Thailand, and Africa, the narratives we examined demonstrated the detrimental effect of lymphedema of the leg on psychological and emotional well-being $(3,8,9,23,24)$. While some variation was expressed, women in this study described similar underlying negative psychological states, emotional reactions, and cognitive responses in the interviews. Thematic experiences associated with the condition appeared consistent despite the stage of lymphedema and included embarrassment and shame; sadness, depression, and fear; personal losses; pity and social isolation; stigma; and despair.

In keeping with stress-related research, we found that psychological distress results when women determine that they have insufficient resources, both physical and psychological, to meet the challenges of their personal situations $(25,26)$. This person-environment dissonance experienced by women with lymphedema, as noted by the themes that emerged in their narratives, was multi-dimensional.

Women in our study often expressed feelings of self-consciousness, ill ease, or shame about the appearance of their legs. In advanced stages of lymphedema a woman's leg can be swollen to very large proportions and have open wounds; moist, deep folds; and unsightly fungus which can even attract flies. Even in moderate cases, women often can't wear shoes. The inability to wear shoes often prevented women from attending social activities and fulfilling expected cultural roles and responsibilities. Some women perceived pity and discrimination when in social situations and actively grieved over their loss of normalcy. The disfigurement of the leg negatively influenced body image, self-esteem, and social identity among many women. Experiences of enacted stigma and discrimination from others as well as women's experiences of perceived stigma, in which they anticipated discriminatory behaviors from others, often contributed to social isolation $(27,28)$.

Social isolation has been shown repeatedly to prospectively predict mortality and serious morbidity, both in the general population and in individuals with other chronic diseases (29). Evidence from previous studies also suggests that women's self-esteem is linked to their social roles, relationships, and connections to others (30). Many Latina women in a traditional culture are often seen as the primary family nurturers, with strong communal connections and responsibilities in church and community (31). It is, therefore, not surprising that many women in our study described feeling socially isolated due to their loss of work, friends, social activities, and social identity, which in turn led to an overall loss of purpose in life and lower self-esteem. Although some women were socially isolated by others, other women isolated themselves in order not to experience shame, embarrassment, and stigma. Thus the loss of social connectedness and social identify appears to be a major contributor to low self-esteem and psychological distress among women in this study.

While psychological distress was ubiquitous in the lives of women in this study, the level, type, and amount of distress appeared to vary over time, and the association does not appear directly related to advancing stage of lymphedema. Some researchers hypothesized that people experiencing threats to their self-esteem will 
engage in social comparison with others similar to them as a mode of self-evaluation (32). Some women engaging in upward social comparison may feel worse about their situation because they see others better off than themselves or those who cope more effectively with their condition (32). We often found that women with less severe disease were more emotionally volatile and distressed than women with severe disease. Women with less severe disease may be more anxious about the future than women who have already experienced progressive lymphedema and developed adaptive coping strategies.

Aging coupled with psychological distress and progressing disease appears to create a highly vulnerable population of women. As women age, children often move away and spouses die, weakening support networks. For some women, the stress of repeated failure to find a cure combined with the loss of family and social support led to perceived loss of control and hopelessness, thus promoting the phenomena of learned helplessness (33). The phenomenon of learned helplessness was seen in depressed women who had expressed hopelessness and suicidal thoughts at some point in their life.

\subsection{Coping processes and strategies}

Despite the high prevalence of psychological distress expressed throughout the narratives, most women had devised adaptive coping strategies. Coping is a dynamic process of managing stressful (internal and external) situations, changing over time, with resources and support that may also be changing (26). Like other studies, we saw that internal resources of personality and coping methods and external resources of money, education, and social support influenced coping abilities (25). Women in this study used problem-solving coping strategies by purposefully and exhaustively seeking care for their legs. They also used emotional coping strategies, seeking out family, friends, and social networks to regulate the emotional consequences of dealing with the sequelae of disease (26). Downward social comparison allowed some women to positively reframe their situations by seeing themselves as better off than those with more distressing conditions (32). Some women used distancing to cope with their condition. This strategy allows people to detach themselves from a situation. Many women used hope as they continued to look to the future for a miracle. Previous research has suggested that the idea of hope is critical in the Dominican culture (34).

Researchers studying the biobehavioral responses of women to stress now posit that women respond to stress differently than men and characterize this response as "tend and-befriend" $(35,36)$. They assert that affiliation with others and the seeking of social contact during stress is an adaptive response and may depend on an underlying biological mechanism whereby oxytocin, a stress hormone modulated by estrogens, contributes to this affiliative response $(35,36)$. We found that many women in this study indeed turned to and depended upon their families, friends, social, and church networks. Some women spoke about the special affiliation with other women, who were able to provide primary support in ways that men could not. Some women described their role as parent and nurturer as giving them the strength to live with lymphedema. 
Faith and religion were often significant forces in women's cache of coping strategies. Similar to findings from other studies on the role of religion in coping with disability, we found that many women with lymphedema find inner strength from faith to rise above physical and psychological distress (37-39). Research has also shown that a woman's sense of self is often drawn from her connectedness to others. During stressful experiences, women often seek affiliation with other members of their faith (39). In the Dominican Republic the concept of hope is often derived from one's faith in God and supported by relationships, resources, and work (34). Thus, hope along with social support from family, friends, and fellow religious followers appear to give many women the power to transcend the conditions they face. Hope may also help women to cognitively reframe their situation in a more positive manner.

\subsection{Programmatic implications}

Program managers in developing countries with few resources are seldom able to assess and strategically plan for the psychological distress associated with chronic conditions such as lymphedema. By understanding the range of consequences associated with the disease and the resulting coping mechanisms that have successfully served women, we can better inform the programmatic decision-making processes for developing gender-sensitive lymphedema management interventions (40). Identifying and educating women in disease management, early in the course of disease would forestall many of the physical, functional, social, and economic consequences of disease that contribute to psychological distress.

For women who have already developed clinical manifestations of lymphedema, a family-oriented intervention would allow for maximizing the limited resources available to patients and their families. Family-oriented interventions, as we have seen in other chronic illnesses, would encourage physical and psychological support in a more structured manner (41). Educating family members in the disease process, training them in lymphedema management activities, and encouraging them to provide emotional support for women could maximize limited program resources. The affiliative responses of women to stress suggest that culturally tailored support groups could also be a viable behavioral intervention. Women could be offered a chance to participate in a psycho-educational group setting with other similar women sharing positive coping strategies, providing nonjudgmental emotional support, and offering reinforcement for home-based self-management activities to slow progression of lymphedema. The role of faith for the women in this study suggests that creating faith-based lymphedema management initiatives that collaborate with local churches and religious organizations may provide supplemental emotional and social services support for women. The role of hope in the culture of care among these women suggests the need for interventions that allow women to assess their situations openly while acknowledging hope and confronting hopelessness. Developing gender-sensitive instruments that could measure physical and mental health across stages of disease within the cultural context of women with this disease would allow program personnel to evaluate and, when appropriate, refer women for additional medical care. 


\section{Limitations}

The women we interviewed were broadly representative of those women with lymphedema of the leg in the filariasis-endemic, rural, peri-urban, and urban communities in the south coastal region of the Dominican Republic. Some caution may be needed when translating these findings to women in more rural areas with less education and fewer resources. Our sample size, typical of qualitative research, was small, and the accounts presented here may not reflect all women's experiences. While there was high psychological distress expressed by this sample of women, no standardized psychological testing was conducted that could diagnostically label them with depressive or other psychiatric conditions.

\section{Conclusions}

In the narratives we analyzed, women across all stages of lymphedema described not only the detrimental psychological effect of lymphedema but also positive adaptive coping strategies to help them with the psychological sequelae. While many women described meaningful experiences and adaptive coping strategies for dealing with their distress, it would be a mistake to underestimate the degree of suffering that they experienced. Many women with lymphedema, already in vulnerable situations due to poverty, poor nutrition, and progressing disease, face a future of psychological distress complicated by aging that can render them socially isolated and less able to cope with these adverse circumstances. Further research is required to 1) understand more fully the depth of psychological distress in women; 2) identify instruments that can measure physical and psychological health across stages of disease and within the cultural context of women with this condition; 3) create, teach, and conduct training sessions for health providers and lymphedema management program staff on the psychological sequelae of the condition, potential positive coping strategies, and to establish referral mechanisms for mental health support as needed; and 4) develop, implement, and evaluate family-oriented intervention strategies, support groups, and faith-based programs to minimize psychological sequelae among women with lymphedema in filariasis-endemic countries. 


\section{References}

1. US Department of Health and Human Services. Lymphatic filariasis in the Americas: an epidemiologic history. Atlanta, GA: Centers for Disease Control and Prevention; 2002.

2. Babu BV, Nayak AN, Acharya AS, Jangid PK, Mallick G. The economic loss due to treatment cost and works loss to individuals with chronic lymphatic filariasis in rural communities of Orissa, India. Acta Tropica. 2002;82:31-8.

3. Kumari A, Krishna, Harichandrakumar KT, Das LK, Krishnamoorthy K. Physical and psychosocial burden due to lymphatic filariasis as perceived by patients and medical experts. Trop Med Int Health. 2005;10:567-73.

4. McPherson T, Penzer R. A comparison of quality of life and disease severity in 54 patients with lymphedema in Guyana. Br J Dermatol. 2003;149(Suppl 64):34.

5. Ramaiah KD, Guyatt H, Ramu K, Vanamail P, Pani SP, Das PK. Treatment costs and loss of work time to individuals with chronic lymphatic filariasis in rural communities in south India. Trop Med Int Health. 1999;4:19-25.

6. Ramaiah KD, Kumar KNV, Ramu K, Pani SP, Das PK. Functional impairment caused by lymphatic filariasis in rural areas of South India. Trop Med Int Health. 1997;2:832-8.

7. Bandyopadhyay L. Lymphatic filariasis and the women of India. Soc Sci Med. 1996;42:1401-10.

8. Bernhard P, Makunde RW, Magnussen P, Lemnge MM. Genital manifestations and reproductive health in female residents of a Wucheria bancrofti-endemic area in Tanzania. Trans R Soc Trop Med Hyg. 2000;94:409-12.

9. Coreil J, Mayard G, Louis-Charles J, Addis D. Filarial elephantiasis among Haitian women: social context and behavioral factors in treatment. Trop Med Int Health. 1998;3:467-73.

10. Epping-Jordan J. The challenge of chronic conditions: WHO responds. BMJ. 2001;323:947-8.

11. Central Intelligence Agency. Dominican Republic. The World Factbook. 29 March 2006. Available at: http://www.cia.gov/cia/publications/factbook/geos/dr.html. Accessed April 5, 2006.

12. Vincent AL, Gonzalvo A, Cowell BC, Nayar JK, Uribe L. Research notes: a survey of bancroftian filariasis in the Dominican Republic. The Journal of Parasitology. 1987;73: 839-40.

13. Berg B. Qualitative research methods for the social sciences. London: Allyn and Bacon; 2001.

14. Madriz E. Focus groups in feminist research. In: Denzin NK, Lincoln YS, editors. Handbook of qualitative research. Thousand Oaks, CA: Sage Publications; 1994:835-50.

15. Patton MQ. Qualitative interviewing. In: Qualitative research and evaluation methods, 3rd ed. Thousand Oaks, CA: Sage Publications; 2003:339-427.

16. Glaser B, Strauss A. The discovery of grounded theory: Strategies for qualitative research. Chicago: Aldine; 1967.

17. Strauss A, Corbin J. Basics of qualitative research: techniques and procedures for developing grounded theory. Thousand Oaks, CA: Sage Publications; 1998.

18. Watters JK, Biernacki P. Targeted sampling: options for the study of hidden populations. Social Problems. 1989;36:416-30.

19. Dreyer G, Addiss D, Dreyer P, Noroes J. Basic lymphedema management: treatment and prevention of problems associated with lymphatic filariasis. Hollis, NH: Hollis; 2002.

20. Patton MQ. Designing qualitative studies. In: Qualitative research and evaluation methods. 3rd ed. Thousand Oaks, CA: Sage Publications;2003. p. 230-47.

21. Muhr T. ATLAS-ti version 4.2 [computer software]. Berlin: Scientific Software; 1997.

22. Miles MB, Huberman AM. Qualitative data analysis. Thousand Oaks, CA: Sage Publications; 1994.

23. Rauyajin O, Kamthornwachara B, Yablo P. Socio-cultural and behavioral aspects of mosquito-borne lymphatic filariasis in Thailand: a qualitative analysis. Soc Sci Med. 1995;41:1705-13. 
24. Gyapong M, Gyapong JO, Adjei S, Vlassoff C, Weiss M. Filariasis in northern Ghana: some cultural beliefs and practices and their implications for disease control. Soc Sci Med. 1996;43:235-42.

25. Lazarus RS, Folkman S. Stress, appraisal, and coping. New York: Springer; 1984.

26. Taylor SE. Stress. In: Health Psychology. 5th ed. New York: McGraw-Hill; 2003:178215.

27. van Brakel WH. Measuring health-related stigma-a literature review. Psychol Health Med. 2006;11:307-34.

28. Goffman E. Stigma: notes on the management of spoiled identity New York: Simon \& Schuster; 1963.

29. Brummett BH, Barefoot JC, Siegler IC, Clapp-Channing NE, Lytle BL, Bosworth HB, et al. Characteristics of socially isolated patients with coronary artery disease who are at elevated risk for mortality. Psychosomatic Medicine. 2002;63:267-72.

30. Josephs RA, Markus HR, Tafarodi RW. Gender and self esteem. J Pers Soc Psychol. 2002;63:391-402.

31. Baud M. Patriarchy and changing family strategies: Class and gender in the Dominican Republic. The History of the Family. 1997;2:355-77.

32. Suls J. Contributions of social comparison to physical illness and well-being. In: Suls J, Wallston $\mathrm{K}$, editors. Social psychological foundations of health and illness. Oxford: Blackwell; 2003. p. 226-55.

33. Seligman MEP. A learned helplessness point of view. In: Rehm L, editor. Behavior therapy for depression. New York: Academic Press; 1980. p. 123-42.

34. Holt J, Reeves JS. The meaning of hope and generic caring practices to nurture hope in a rural village in the Dominican Republic. J Transcult Nurs. 2001;12:123-31.

35. Taylor SE. The tending instinct: women, men, and the biology of our relationships. New York: Times Books; 2002.

36. Taylor SE, Klein LC, Lewis BP, Gruenewald TL, Gurung RAR, Updegraff JA. Biobehavioral responses to stress in females: tend-and-befriend, not fight-or-flight. Psychol Rev. 2000;107:411-29.

37. Boswell BB, Knight S, Hamer M, McChesney J. Disability and spirituality: a reciprocal relationship with implications for the rehabilitation process. J Rehabil. 2001;67:20-5

38. Ellison CG, Levin JS. The religion-health connection: evidence, theory, and future directions. Health Educ Behav. 1998;25:700-20.

39. Nosek MA, Hughes R.B. Psycho-spiritual aspects of sense of self in women with physical disabilities. J Rehabil. 2001;67:20-6.

40. Rathgeber EM, Vlassoff C. Gender and tropical diseases: a new research focus. Soc Sci Med. 1993;37:513-20.

41. Teufel-Shone NI, Drummond R, Rawiel U. Developing and adapting a family-based diabetes program at the US-Mexico border. Preventing Chronic Disease [serial online]. Jan 2005. Available at: http://www.cdc.gov/pcd/issues/2005/jan/04_0083.htm. Accessed April 3, 2006. 


\title{
Chapter 4
}

\section{Health-Seeking Behaviors and Self-Care Practices of Dominican Women with Lymphedema of the Leg: Implications for Lymphedema Management Programs}

\author{
Bobbie Person, David Addiss, L. Kay Bartholomew, Cecilia Meijer, Victor Pou, \\ and Bart van den Borne
}

Manuscript published as: Health-seeking behaviors and self-care practices of Dominican women with lymphoedema of the leg: implications for lymphoedema management programs. Filaria Journal. 2006;5:13. 


\section{Abstract}

In the Dominican Republic, a Latin American country with filariasis-endemic areas, more than 63,000 people have lymphatic filariasis and more than 400,000 people are at risk of future infection. In this paper, we explore the health beliefs, health-seeking behaviors and self-care practices of women with lymphoedema in filariasis-endemic areas to better understand the needs of women when developing lymphoedema morbidity control programs. Qualitative data were collected through semi-structured interviews of 28 women, 3 focus group discussions with 28 women, field notes and photographs. Women described exhaustive and expensive attempts at seeking a cure for their lymphoedema. Family members were influential in providing women with initial care seeking referrals to indigenous healers credited with influence over physical, mental, spiritual and supernatural properties of illness. When indigenous treatments proved to be ineffectual, the women sought care from trained healthcare providers. Most healthcare providers incorrectly diagnosed the edema, failed to adequately treat and meet the needs of women and were viewed as expensive. Most women resorted to self-prescribing injectable, oral, or topical antibiotics along with oral analgesics as a standard practice of self-care. Healthcare providers must understand a woman's cultural perspectives of illness, her natural networks of support and referral, her behavioural practices of care-seeking and selfcare and the financial burden of seeking care. In the culture of the Dominican Republic family members and traditional healthcare providers are influential advisors on initial health-seeking behaviors and self-care practices. For this reason family-oriented interventions, support groups for women and their families, community education, and training on simple, low cost lymphoedema management techniques for indigenous healers are viable ways to influence the early detection, diagnosis and treatment of women with lymphoedema. The extensive use of injectable, oral and topical antibiotics by indigenous healers and women without medical supervision suggests a need for health education messages related to the risks of such practices. 


\section{Introduction}

The global prevalence of lymphatic filariasis, a mosquito-transmitted parasitic disease, is vast (1). In the Americas alone an estimated 8 million people are affected (2). In the Dominican Republic more than 63,000 people have lymphatic filariasis and more than 400,000 people are at risk of future infection (3). In 1998, a global initiative aimed at eradication of the disease by the year 2020 was established (4). This initiative, the Global Programme to Eliminate Lymphatic Filariasis (GPELF), was built upon the twin pillars of interruption of transmission through mass drug administration and morbidity control (5). The Dominican Republic has initiated both of these strategies in the filariasis-endemic areas of the country, which include parts of the rural southwest and the capital city, Santo Domingo. In different endemic regions researchers have begun to look more closely at the quality of life issues related to the behavioural, economic and social consequences of lymphoedema to inform lymphoedema management programmes about the needs of those affected by this condition (6-11).

In Latin America, however, few have examined the complex relationship between health-seeking behaviors, self-care practices and the cultural context of these behaviors among women who live with chronic manifestations of disease (12). Lymphoedema management requires compliance with a prescribed, simple regime of self-care practices including leg hygiene, elevation, exercise, and self- examination with self-referral for appropriate medical care as needed $(13,14)$. Healthcare providers should understand a woman's cultural perspectives of illness, her natural networks of support and referral and behavioural practices of care seeking and selfmanagement that she believes support her health (15). Optimal care should also be informed by understanding the financial, psychological, and emotional costs associated with health-seeking that women face. The purposes of this qualitative paper were 1) to explore specific health beliefs, health-seeking behaviors and selfcare practices specific to women with lymphoedema in filariasis-endemic areas of the Dominican Republic, about which little is known; and 2) to provide recommendations for morbidity control programmes for lymphoedema management.

\section{Materials and methods}

\subsection{Study area}

The Caribbean island of Hispaniola comprises two filariasis-endemic countries, the Republic of Haiti and the Dominican Republic (16). Researchers in the Dominican Republic have documented lymphatic filariasis in the poor, densely populated urban neighborhoods of the cities called barrios and in the poor rural communities associated with sugarcane production in the southern municipalities called bateys (17). While there are no data specific to Dominican women, a study in 1998 documented transmission among school children in parts of both study areas, 
the Barrio La Cienaga in Santo Domingo, with a microfilaremia prevalence of $12.8 \%$ (29/227), and Barahona City, with a microfilaremia prevalence of $4.1 \%(10 / 243)(18)$.

\subsection{Qualitative data collection methods}

These data were collected as part of a larger study on Dominican women with lymphedema that explored the impact of disease on multiple domains of life, economic vulnerability, health seeking behaviors, and social support. Qualitative methods were used to gather data for this topic because little is known about the beliefs, values, norms, traditions, roles, experiences, and understanding of the disease process by women with lymphoedema in the Dominican Republic. Qualitative techniques are especially relevant in areas where there has been little previous research and there is a need for discovery, descriptive accounts, and an understanding of how people interpret and explain a social phenomenon or personal experience (19). Data were collected through semi-structured interviews, focus group discussions (FGD), field notes, and photographs of women from January 2002 to October $2004(20,21)$. A semi-structured interview guide was used to encourage women to discuss their perceptions of illness, healthcare sought from indigenous healers and trained physicians, medical treatments, results of treatment, costs of treatments, and self-management of their lymphoedema. Questions were translated into Spanish, then pretested on a sample of local women with lymphoedema and modified to correspond with local dialect and cultural meanings before use in the interview guide. A bilingual research assistant back-translated the guide into English to ensure congruence with the original questions. Other women with lymphoedema participated in 3 focus groups. These groups discussed new and emerging ideas that arose from the in-depth interview data. Focus group data along with researcher notes served in triangulating the data. Questions in the FGD guide encouraged women to discuss in detail their attempts to seek a cure, types of traditional care sought, experiences of seeking care from trained physicians and their current regime of care.

We used modified precepts of grounded theory, an inductive method that elicits theory from the data, moving from the specific to the more general perspective (22). Ideas emerging from the data guide gave the researcher new ideas to explore as they arose during the interview and across interviews. The constant comparisons of themes within and between interviews specifically informed and guided data analysis.

\subsection{Sampling and recruitment}

Researchers used a targeted sampling methodology to recruit women with lymphoedema of the leg (stages 3-7 based on the staging criteria of Dreyer et al.) who lived in filariasis-endemic areas, participated in no prescribed lymphoedema management activities, or were inconsistent with lymphoedema management activities and agreed to be interviewed, audiotaped and photographed $(13,23)$. Based on the staging criteria of Dreyer (13), permanent (non-reversing) lymphoedema is considered Stage 3 if shallow skin folds are present; Stage 4 if there are protrusions or "knobs"; Stage 5 if deep skin folds are present; Stage 6, if "mossy lesions" are present; and Stage 7, inability of patient to perform activities of daily living. 
In 2003, the Centro Para el Control de Enfermedades Tropicales (CENCET) conducted a lymphatic filariasis census in the southern, filarial-endemic region of the country prior to the administration of a programme of mass drug distribution for the interruption of transmission of disease (3).The census data, collected by trained health workers, identified any woman with observable lymphoedema of the leg. Using this data public health officials referred women in the community with observable lymphoedema to local dermatology clinics associated with the Instituto Dermatológico de Cirugia y Piel (IDCP) in the coastal towns of Santo Domingo and Barahona. Dermatologists in each clinic screened and then referred women who met the study criteria to be interviewed prior to their being instructed in the care and management of their lymphoedema. Of approximately 70 women that were identified, 30 women meeting the screening criteria were referred to us for an indepth interview. One woman refused to be interviewed and one interview tape was damaged. The 28 in-depth interviews, each approximately 2 to 3 hours long, took place in either the homes of the women or in the dermatology clinic before a lymphoedema management session

Twenty-eight other women each participated in a 2-hour FGD at IDCP clinics; two such groups met in Santo Domingo and one group met in Barahona. Women who participated in the FGDs met the same criteria as did the women who were interviewed, although a few of the women in the FGD had previously received an educational session on lymphoedema management during prior clinic visits and were practicing the prescribed regime of care. Any woman coming into the dermatology clinic during the 2-week period prior to our visit, who had not been previously interviewed, was invited to participate in a FGD and the first eight women who agreed were accepted. If a woman cancelled, clinic staff called another woman from the clinic that met the screening criteria and asked her to participate. While women in the FGD generally did not know each other the similarity of their condition and their lives allowed them to bond during discussions which provided a safe space for expressing their feelings and experiences.

\subsection{Informed consent}

The institutional and ethical review boards of CENCET and the US Centers for Disease Control and Prevention (CDC) approved the study protocol, which required informed consent from each study participant.

\subsection{Data management and analysis}

Semi-structured interviews and FGDs conducted in Spanish were audiotaped and transcribed verbatim. The transcripts were translated into English by a bilingual research assistant and then entered as a Microsoft Word document into ATLAS-ti to facilitate data coding, text searching and analysis (24). Data was systematically examined for emerging codes, themes, and patterns. To ensure inter-coder agreement, two coders derived open coding schemes independently on a subset of interviews, then made comparisons to derive consensus on the coding strategy and repeated the coding independently on another subset of interviews to ensure consistency of the implementation of the coding strategy (25). Themes related to the 
perceptions of illness, health-seeking behaviors associated with receiving care from indigenous or scientifically trained healthcare providers, treatments and treatment effects, self-management of disease, cost and cultural practices emerged as most salient to the women. Through axial coding and developing thematic tables and matrices, we were able to extract the direct quotes of women, categorize the quotes into codes and then the codes into categories and themes that women discussed, and then analyze any relationships we found among the data $(22,25)$. Consensus between the principal investigator and the research assistant was required in the interpretation and meaning of the chosen texts of women. When a meaning was not clear, we discussed the text segment and sought opinions with colleagues for clarification.

\section{Results}

\subsection{Demographic description}

Sixty-seven percent of the 56 women in the study were older than 40 , with an average age of 50.5 and an age range of $26-80$. Of the 56 women participating in the study, $40 \%$ had lymphoedema in each leg, $36 \%$ in just the left leg and $24 \%$ in just the right leg. Approximately $64 \%$ of women had severe lymphoedema, stages 4-6. Data from only the in-depth interviews of 28 women revealed that $46 \%$ were currently heads of households; 71\% were married or in a free union or common-law marriage, $18 \%$ were widowed and $11 \%$ were single. Seventy-eight percent of the women could read and write. Ninety-four percent of women who were interviewed had sought care for chronic lymphoedema, with $96 \%$ seeking care from traditional providers and $90 \%$ seeking care from trained physicians at some time during the course of their disease. Seventy-two percent had sought care during episodes of acute adenolymphangitis. Of the other 28 women who participated in the FGDs, all had sought care for chronic lymphoedema and acute adenolymphangitis from both indigenous healers and scientifically trained physicians. Women in the study were from rural, semi-rural, and urban areas where mass drug distribution to interrupt transmission and lymphatic filariasis educational campaigns had taken place, but while many had taken the medications they usually did not associate the campaign with their condition.

\subsection{Local terminology and perceptions of causation}

Few women in the in-depth interviews were able to answer questions correctly about the etiology of their lymphoedema and its association with lymphatic filariasis. Approximately half of the women in the FGDs talked about the association of lymphoedema with filariasis. Women in the FGDs also described seeing a recent campaign associated with mass drug distribution for the interruption of transmission of disease. Additionally, some of the women in the FGDs described receiving instruction in managing their lymphoedema, which included a discussion about transmission of lymphatic filariasis and the cause of lymphoedema.

The women in this study had several local names for the lymphoedema including, erisipela or dicipela. A few of the older women had heard the condition 
called milk leg. Women called an episode of acute adenolymphangitis a crisis, which was often preceded by a seca, or swelling of the inguinal lymph nodes. A seca might also be a large boil in the groin or upper thigh that often precedes or accompanies a crisis. Spider bites, pregnancy, menopause, heredity, varicose veins, accidents and magical or supernatural events were common explanations for lymphoedema. Several women blamed individual children for their lymphoedema because of it's onset during their pregnancy. Many women with more severe lymphoedema attributed the onset of a crisis to having a wetness or a fungus between the toes, getting a cut on the leg, getting the foot wet or dirty, stubbing one's toe, working too hard or walking too far, or wearing underwear that is too tight and causes a seca. Women with stage 3 , less severe lymphoedema, often reported not knowing what caused their crisis and often attributed it to supernatural causes such as having small birds in the leg or having a "hex" put on them.

\subsection{Healthcare-seeking behaviors}

"If I tell you all the people I have seen for this leg, it is not going to fit there on your paper," said a woman with severe lymphoedema. Women described exhaustive attempts at seeking treatments and a cure for their lymphoedema; they visited traditional herbalists, ensalmadores (specialized herbalists), curanderos (healers), brujos (witchdoctors), as well as trained physicians to try to find relief. Women sought care for a crisis of acute swelling, high fever, headaches, nausea, and pain, as well as the more chronic manifestations of the condition such as irreversible swelling and disfigurement, open wounds and boils, large skin folds, fungal infections and disagreeable odor. Despite the high prevalence of psychological distress described by most women, with a few women even having contemplated suicide at some point in their lives, no one described seeking care specifically for their psychological distress.

\subsubsection{Seeking Healthcare from Indigenous Healers}

Most women described the first or second episode of acute adenolymphangitis as the impetus for first seeking care from an indigenous healer. Family members, acting as lay health advisors, served a critical role in positing the initial cause of lymphoedema as well as offering a referral to an indigenous healer. A few women told us that, while a family member may have taken them to an indigenous healer when they were children, they no longer sought them out because it conflicted with their Christian beliefs or they now viewed them as quacks. The indigenous healers women initially consulted to treat their lymphoedema included traditional herbalists, ensalmadores, curanderos, and brujos. Community members were also another source of advice on how to care for the leg or who to visit for a cure. Women most often described being ensalmed. Ensalmadores are people who have special techniques aimed at curing erysipelas or lymphoedema, including recitation of incantations and prayers to defeat supernatural powers that cause the condition, as well as the application of herbal preparations to the leg. The women also visited curanderos who addressed physical, spiritual, and mental anguish by consulting with saints in order to see which herbs, roots and various home cures to employ to dispel bad magic. Traditional herbalists prescribed bottles of liquid steeped with special herbs to drink, rub on the leg, and/or use as an enema as treatments for chronic 
lymphoedema. Brujos were most commonly used to rid the body of malevolent spirits that at times can take over the individual being treated. If one wanted to remove a "hex" or remove a spell they would visit a brujo. Several women described traveling to Haiti to seek a cure. Several women said that it was well known that this condition is prevalent in Haiti and the indigenous healers have more experience removing spells or bad magic. Women who had sought care from indigenous healers in Haiti were older, presented with the severest lymphoedema and had at some time in their life lived near the Haitian border.

Women described more than 65 treatments for lymphoedema of the leg suggested by indigenous healers, family members and friends. Recommended herbal remedies included prepared teas using local herbs and leaves, prepared creams, poultices of papaya and leaves, and the application of higuereta, a derivative of the castor bean, to the leg. Women also described using leeches, pricking the leg to drain it, soaking it in hot water, and pouring kerosene on the leg. Other treatments included sweeping the leg with a small broom, burying the leg in the ground, walking in salt water in the sea and rubbing the belly of a frog across the leg. Ensalmadores, curanderos, and brujos often prescribed injectable medications bought at the local pharmacy to supplement their traditional treatments for lymphoedema. A middleaged woman said, "When the lady ensalmed me the first time, she gave me some cream to put on my leg that night and the swelling of the leg got smaller because it was very affected. In addition, she had me take a penicillin injection. It cost twentyfive peso. She injected it in to my backside."

Women described some of the traditional treatments as having benefits or at least doing no harm. Poultices, such as papaya, and teas offered women some relief during a crisis when the leg was very hot and swollen and women experiencing severe headaches and nausea. However, many traditional treatments provided no relief for most women and some, such as pouring kerosene on the leg or attempting to drain the leg, exacerbated their condition. "My family wanted me to be cured, so my mother took me to see many brujos and healers. I almost died because of one of those old men," said an older woman with severe lymphoedema.

The cost of traditional treatments ranged from no cost to substantial sums. A friend of the family might ensalm a woman for no money but barter a bottle of whiskey, a chicken, or another commodity. The women cited the cost of a single visit to an indigenous healer as ranging from $100-500$ pesos (\$2-10 USD) and herbal preparations, teas, creams, and other items were an additional expense. Injections bought at a local pharmacy were relatively inexpensive and ranged from 15-125 pesos ( $\$ 0.20-2.50$ USD). The women described the need for multiple sessions with multiple healers and the cost for these sessions ranged from 1,500-5,000 pesos (\$30-100 USD). A woman seeking a cure said, "I have gone to so many people and spent so much money with this illness. It has taken away from me more than 60,000 pesos ( $\$ 1,600$ USD), and I have not seen any improvement." Women often required financial assistance from family members to pay their treatment costs.

We had informal discussions with one ensalmadore and one curandero neither of which would discuss in-depth their healing techniques but did share some general information with us. The ensalmadore was well known in the community. $\mathrm{He}$ described a general regime of hygiene and elevation that would be in keeping with 
recommended prescribed leg hygiene and elevation. Additionally, the ensalmadore described ritual prayers and conducted special incantations over the leg while applying traditional herbs to the leg. He also told us that when a woman's leg got very, very big the swelling would never go away and then it would then be in God's hands. The curandero suggested that a woman dig a hole in the ground and bury her leg in dirt on a regularly prescribed timeline. The woman would then have to bring an offering and pray to a specific religious saint every Friday. The curandero also prescribed specific tonics for enemas. The ensalmadore and the curandero required a payment of money and other goods for their treatments.

\subsubsection{Seeking Healthcare from Trained Physicians}

Some women described seeking care from a medically trained physician and an indigenous healer at the same time. However, most women described seeking out trained physicians only after their attempts to treat their lymphoedema with indigenous treatments or home remedies failed. The women in our study described exhaustive attempts to find a physician who could treat their condition.

A woman with lymphoedema for more than 10 years said, "I am so tired and I have spent so much on doctors and more doctors. Many doctors have treated me but no one has helped. They just take my money."

Women sought care from a variety of physicians including those trained as general practitioners, cardiovascular surgeons, orthopedic surgeons, oncologists, acupuncturists, chiropractors, and dermatologists. Some physicians ran tests for lymphatic filariasis but when the tests came back negative they told the women that they did not know the cause of their lymphoedema. Many physicians incorrectly diagnosed the condition as varicose veins, cancer of the leg and afflictions of the spine. Physicians prescribed diuretics, analgesics, antipyretics, anti-inflammatories and antibiotics including ampicillin and penicillin (usually as an injectable). Physicians rarely gave women adequate explanations as to the cause of their lymphoedema or the purpose of their medications. However, many women saw positive changes when using antibiotics. Several women we interviewed had surgery to "unclog" their veins and reduce the size of their legs. The results left women with severe scarring and further disfigurement with no impact on their chronic lymphoedema or acute episodes of adenolymphangitis. In addition, the cost was substantial (up to $\$ 6,000$ USD). Two women reported being told by their physician that their leg should be amputated, with one physician telling a woman that the lymphoedema was the result of cancer of the leg. Physicians rarely asked if women were receiving care from any indigenous healers and if women self-disclosed that information they were often ridiculed by the physician.

Women who saw no effect from the prescribed ointments, creams and pills their physicians prescribed were often distraught over the cost of care and the burden it caused their families. Most women agreed that antibiotic injections were the most successful treatment to stave off or treat a crisis. Women, individually and in the focus groups, described injections of antibiotics as the best treatment for boils, abscesses and other wounds on the leg. However, they expressed disappointment that antibiotics did not provide a cure for their chronic swelling. Almost two-thirds of the women described buying injectable antibiotics from local pharmacies and self- 
injecting or having a family member or neighbor inject them without physician supervision. One woman who had recently had an episode of acute adenolymphangitis said, "The doctor solves everything with a prescription and I know he will give me a penicillin injection. So now I just do it myself. I don't go to the doctor anymore."

Similar to the cost of traditional treatments, physician fees and medications ranged from no cost to substantial sums. Social security welfare visits were sometimes free and occasionally a woman would get a reduced cost because one of her family members worked at a healthcare facility. One focus group participant said, "They gave me a reduced cost because my husband worked there. The visits to the hospital came out to be about 4,00o pesos ( $\$ 80$ USD) each."

Consultations with private physicians and clinics ranged from 200-1,000 pesos (\$4-20 USD) and often required multiple visits with medical tests adding to their fees. Women described their medicines as expensive, ranging from 150-3,600 pesos (\$3-72 USD). Injections bought at a local pharmacy were less inexpensive than those purchased from a physician. A woman with severe lymphoedema talked about the costs of her medications: "I have to inject myself again with penicillin and buy a pain reliever. It ends up costing me 2,000-3,000 pesos to buy from the doctor. It's much, much cheaper to just buy the medicine myself."

Several women in the FGDs were currently participating in a lymphoedema management programme of prescribed hygiene, exercise and elevation with medications as needed. These women described positive interactions with dermatologists trained in simple, low cost lymphoedema management techniques. They described feeling better informed and more hopeful because physicians and support staff showed more concern for them. These women were pleased with the care they were receiving and were able to describe the cause of lymphatic filariasis, the cause of lymphoedema and techniques to care for their leg on a daily basis as well as during a crisis. Women were pleased with the healing of skin lesions and reduction of crisis but still disappointed at the lack of a cure.

\section{Discussion}

In the Dominican Republic, as in other Caribbean and Latin American cultures, traditional healthcare providers and indigenous healers are an integral part of physical, mental, and spiritual healthcare for many people $(26,27)$. Researchers estimate that more than 3,000 indigenous healers, along with 16,000 physicians, practice in the Dominican Republic $(28,29)$. Currently, the extent and significance of treatment of lymphoedema by indigenous healers, as well as by trained physicians, are poorly documented. Indigenous healthcare beliefs and practices are the result of settlement of the country by Spanish explorers and later by the tribal customs and beliefs of African slaves (30). This integration of cultures created a mixture of Catholicism, mysticism and other magico-religious means for dealing with life, illness, and death.

Findings from this study indicate that family, friends, and strong cultural beliefs influence women's explanatory model of illness (EMS) (31). Women's EMS significantly influenced their health-seeking behaviors. A personal EMS is distinct from general beliefs about illness and health due to the specificity to explain a 
particular illness event. An EMS is the way a person perceives, mentally represents and culturally defines the episode of illness including the cause, symptoms and explanations for illness, diagnosis, treatments, and cultural role of the ill person (31). A woman's EMS often guides her outcome expectations, as well as her coping strategies and health-seeking behaviors within a socio-cultural context and can change over time as we see in our findings. The reality that lymphoedema is culturally ingrained in the Dominican Republic is evident by the presence of ensalmadores who are described as having special techniques for curing erysipelas or lymphoedema.

Essential to any successful lymphoedema management programme is the understanding of the specific factors by which women interpret and place their illness experiences. The Dominican women in this study often had plausible explanations for their initial episodes of illness and solicited culturally relevant care and coping strategies from family, friends and recommended indigenous healers. Similar to findings from other countries, the current cultural paradigm of seeking indigenous healers as a first-line treatment often resulted in not only a significant delay in appropriate medical treatment but also potentially harmful treatments that could exacerbate the condition (32). Researchers in cultural studies suggest that incorporating interventions within a cultural paradigm is more successful than trying to change a cultural paradigm altogether (15). This theory suggests that providing lymphoedema management training or outreach to indigenous healers such as ensalmadores, as well as encouraging interactions of those individuals with more medically trained healthcare providers could create a vital mechanism for early treatment and referral. Previous public health efforts in Mexico, Ghana, Bangladesh, Zimbabwe and Swaziland have proposed that indigenous healers be incorporated into health systems in various ways, adapting to the specific features of their cultural practice and existing medical facilities and regulations (33-36). Some developing countries are even encouraging regulation and licensing of traditional and indigenous healers and documentation of herbal preparations (36).

Reports from women, that many trained physicians often incorrectly diagnosed and mismanaged lymphoedema, suggests the need for continuing education for practicing physicians and other healthcare providers, as well as incorporation of diagnostic and treatment guidelines into the medical education curriculum. If lymphoedema management programmes are to be successful the need for communication between healthcare provider and patient is essential. Healthcare providers should give clear explanations about factors contributing to the disease, as well as the treatment regime and progressive nature of the condition. It is critical for women to perform self-examinations of the leg and identify potential complications needing a physician's care. When queried, women preferred simple visual instructions with photographs over just written instructions or written instructions with cartoon-like illustrations. A simple pocket-size photographic catalogue of possible conditions such as fungal infections, abscesses and boils could show women what to look for during their self-examinations and then how to treat or self-refer to appropriate healthcare providers.

The pervasive use of family members for emotional and financial support suggests that a family-oriented lymphoedema management intervention could be 
effective. Educating family members in the disease process, training them to manage lymphoedema and encouraging them to provide emotional support for women who have this condition could maximize limited programme resources.

Overall cost of care places a significant burden on the individual and family members. Family-oriented interventions could encourage physical and psychological support in a more structured manner, a method that has worked with other chronic illnesses and allow judicious use of family resources (37).

Researchers studying the bio-behavioural responses of women to stress now posit that women respond to stress differently than men and characterize this response as "tend-and-befriend" (38). They assert that affiliation with others and seeking social contact during stress and illness is an adaptive response with an underlying biochemical basis. In addition, research suggests that support by those who suffer with the same condition is especially valuable because they are seen as more creditable sources of information and there is little shame or stigma in sharing difficulties related to lymphoedema (39). This is in-keeping with previous research which shows that support groups with other women can be a viable tool in providing psycho-educational opportunities with skill building sessions for low cost self-care, reinforcement for self-management efforts as well as emotional support and increased social connectedness for many women (6).

Women in our focus group sessions, who did not know each other prior to the focus group, asked when they could meet again because they shared a sense of connectedness with other women in the group. Additionally, family support groups could be an important strategy for caretakers of those with lymphoedema.

Self-management with antibiotics is a risky practice described by many women in this study. Women do not know what type of infection or other condition they may have, nor if they are injecting or ingesting an appropriate antibiotic to treat their condition. The global appearance of antimicrobial resistance is a multifaceted problem created by the convergence of many factors, including the use and misuse of antibiotics (40). The extensive use of injectable and topical antibiotics by indigenous healers and women without medical supervision suggests a need for treating physicians to educate their patients on the need to follow medically supervised recommendations and to discuss potential risks of unsupervised antibiotic use with them. Indiscriminate use of antibiotics could contribute to antibiotic resistance.

\section{Conclusion}

Similar to other countries, in the culture of the Dominican Republic, traditional healthcare providers, and indigenous healers are often an integral part of physical, mental, and spiritual healthcare for many people. Healthcare providers must understand a woman's cultural perspectives of illness, her natural networks of support and referral, her behavioural practices of care-seeking and self-care that she believes support her health and the financial burden of seeking care.

Training both indigenous healers, considered as lay health advisors by women, along with physicians in simple, low-cost self-care regimes could increase the early detection, diagnosis, and treatment of lymphoedema among women in filariasis-endemic areas of the Dominican Republic. Family-oriented interventions and support groups for women and their families are viable ways to provide psycho- 
educational opportunities with skill building sessions, reinforcement for selfmanagement efforts and emotional support for women. The often indiscriminate use of injectable antibiotics without medical supervision suggests a need for health education messages about this topic for women during lymphoedema management interventions. While these findings are specific to women in the Dominican Republic the methodology of inquiry into local cultural practices and beliefs to tailor interventions are not only applicable but recommended for other countries with lymphoedema management programmes.

\section{Ethical Approval}

Institutional and ethical review boards from CDC and CENCET approved this study. Informed consent was obtained from every participant. 


\section{References}

1. World Health Organization. Expert Committee: lymphatic filariasis: diagnosis and pathogenesis. Bull World Health Organ. 1993;71:135-41.

2. US Department of Health and Human Services. Lymphatic filariasis in the Americas: an epidemiologic history. Atlanta, GA: Centers for Disease Control and Prevention; 2002.

3. Lymphatic Filariasis Elimination in the Dominican Republic. Available at: http:// www.taskforce.org/LFSC/americas/dominican.html. Accessed April 5, 2006.

4. Ottesen EA. The global programme to eliminate lymphatic filariasis (editorial). Trop Med Int Health. 2000;5:591-4.

5. Seim AR, Dreyer G, Addiss DG. Controlling morbidity and interrupting transmission: Twin pillars of lymphatic filariasis elimination. Revista de Sociedade Brasileira de Medicina Tropical. 1999;32:325-8.

6. Coreil J, Mayard G, Addiss D. Support groups for women with lymphatic filariasis in Haiti. Geneva: WHO/TDR; 2003.

7. Ramaiah KD, Vijay Kumar KN, Ramu K, Pani SP, Das PK. Functional impairment caused by lymphatic filariasis in rural areas of south India. Trop Med Int Health. 1997;2:832-8.

8. Gyapong M, Gyapong JO, Adjei S, Vlassoff C, Weiss M. Filariasis in Northern Ghana: some cultural beliefs and practices and their implications for disease control. Soc Sci Med. 1996;43:235-42.

9. Bandyopadhyay L. Lymphatic filariasis and the women of India. Soc Sci Med. 1996;42:1401-10.

10. Coreil J, Mayard G, Louis-Charles J, Addiss D. Filarial elephantiasis among Haitian women: social context and behavioural factors in treatment. Trop Med Int Health. 1998;3:467-73.

11. McPherson T, Penzer R. A comparison of quality of life and disease severity in 54 patients with lymphedema in Guyana. Br J Dermatol. 2003;149(Suppl 64):34.

12. Kanda $\mathrm{K}$. The quality of life among lymphoedema patients due to lymphatic filariasis in three rural towns in Haiti. Tampa, FL: University of South Florida; 2004.

13. Dreyer G, Addiss D, Dreyer P, Noroes J. basic lymphoedema management: treatment and prevention of problems associated with lymphatic filariasis. Hollis, NH: Hollis Publishing Company; 2002.

14. Vagas B, Ryan TJ. Lymphoedema: pathophysiology and management in resource-poor settings-relevance for lymphatic filariasis control programmes. Filaria Journal. 2003,2:4.

15. Pachter LM. Culture and clinical care. Folk illness beliefs and behaviors and their implications for health care delivery. JAMA. 1994;271:690-4.

16. The Central Intelligence Agency. Dominican Republic. The World Factbook. 29 March 2006. Available at: http://www.cia.gov/cia/publications/factbook/geos/dr.html. Accessed April 5, 2006.

17. Vincent AL, Gonzalvo A, Cowell BC, Nayar JK, Uribe L. A survey of bancroftian filariasis in the Dominican Republic. J Parasitol. 1987;73:839-40.

18. Huang S, Paulino F, Gonzálvez G, Dietz V, Stroh G, Addiss D. Elimination of lymphatic filariasis in the Americas: rapid assessment in the Dominican Republic to determine the need for an elimination program. In: The 47th Annual Meeting of the American Society of Tropical Medicine and Hygiene, San Juan, Puerto Rico. Am J Trop Med Hyg. 1998;Suppl:245.

19. Patton MQ. Qualitative interviewing. In: Qualitative research and evaluation methods. Thousand Oaks, CA: Sage Publications; 2002:339-427.

20. Fontana A, Frey JH. The interview: from structured questions to negotiated text. In: Denzin NK, Lincoln YS, editors. Handbook of qualitative research. 2nd ed. Thousand Oaks, CA: Sage Publications; 1994. p. 645-72.

21. Berg B. Qualitative research methods for the social sciences. London: Allyn and Bacon; 2001. 
22. Strauss AL, Corbin J. Basics of qualitative research. 2nd ed. Thousand Oaks, CA: Sage Publications; 1998.

23. Watters JK, Biernacki P. Targeted sampling: options for the study of hidden populations. Social Problems. 1989;36:416-430.

24. Muhr T. ATLAS-ti version 4.2 [computer software]. Berlin: Scientific Software; 1997.

25. Miles MB, Huberman AM. Early steps in analysis. In: Qualitative data analysis. Thousand Oaks, CA: Sage Publications; 1994. p. 50-88.

26. Babington LM, Kelley BR, Patsdaughter CA, Soderberg RM, Kelley JE. From recipes to recetas: health beliefs and health care encounters in the rural Dominican Republic. Journal of Cultural Diversity 1999;6:20-5.

27. Aarons DE. Medicine and its alternatives. Health care priorities in the Caribbean. In: Hastings Center Report, vol 29; 1999. p. 23-7.

28. World Health Organization. Traditional health systems in Latin America and the Caribbean: base information technical project report. Washington, DC: The Organization; 1999.

29. Pan American Health Organization, Country Health Profile for the Dominican Republic. Available at: http://www.paho.org/English/DD/AIS/cp_214.htm. Accessed April 2006.

3o. Nigenda G, Mora-Flores G, Aldama-López S, Orozco-Núñez E. La práctica de la medicina tradicional en América Latina y el Caribe: el dilema entre regulación y tolerancia. Salud Publica Mex. 2001;43:41.

31. Kleinmam A. Core clinical functions and explanatory models. In: Patients and healers in the context of culture. Berkeley, CA: University of California Press; 1980:71-118.

32. Ahorlu CK, Dunyo SK, Koram KA, Nkrumah FK, Aagaard-Hansen J, Simonsen P. Lymphatic filariasis related perceptions and practices on the coast of Ghana: implications for prevention and control. Acta Tropica. 1999;73:251-61.

33. Oppong AC. Healers in transition. Soc Sci Med. 1989;28:605-12.

34. Freeman M, Motsei M. Planning health care in South Africa: is there a role for traditional healers? Soc Sci Med. 1992;34:1183-90.

35. Hoff $W$. Traditional health practitioners as primary health care workers. Tropical Doctor. 1997;27(Suppl 1):52-5.

36. Warren DM, Bova GS, Tregoning MA, Kliewer M. Ghanaian national policy toward indigenous healers. The case of the primary health training for Indigenous Healers (PRHETRIH) program. Soc Sci Med. 1982;16:1873-81.

37. Teufel-Shone NI, Drummond R, Rawiel U. Developing and adapting a family-based diabetes program at the US-Mexico border. Preventing Chronic Disease [serial online]. Jan 2005. Available at: http://www.cdc.gov/pcd/issues/2005/jan/04_0083.htm. Accessed April 3, 2006.

38. Taylor SE, Klein LC, Lewis BP, Gruenewald TL, Gurung RAR, Updegraff JA. Biobehavioral responses to stress in females: tend-and-befriend, not fight-or-flight. Psychol Rev. 2000;107:411-29.

39. Goffman E. Stigma: notes on the management of spoiled identity. New York, NY: Simon \& Schuster; 1963.

40. Smolinski M, Hamburg M, Lederberg J, editors. Institute of Medicine. Microbial threats to health: emergence, detection, and response executive summary. Washington, DC: The National Press; 2003. 


\title{
Chapter 5
}

\section{Disrupted Social Connectedness among Dominican Women with Chronic Filarial Lymphedema}

\author{
Bobbie Person, L. Kay Bartholomew, David Addiss, and Bart van den Borne
}

Manuscript published as: Disrupted social connectedness among Dominican women with chronic filarial lymphedema. Patient Education and Counseling. 2007;68(3):279-86. 


\begin{abstract}
The objectives of this paper were to identify specific factors associated with intact or disrupted social connectedness among Dominican women with chronic filarial lymphedema and better understand the impact of disrupted connectedness on their lives. Data were collected through 28 individual interviews and 3 focus group discussions of 24 women from filariasis-endemic areas of the Dominican Republic presenting with lymphedema of one or both legs. The confluence of chronic and acute stressors with severity of lymphedema led women to rely on others for social support. Women described complications of aging, disability, reduced social networks, and inability to adhere to cultural scripts as contributing to disrupted social connectedness. Social disconnectedness appears to exacerbate the negative consequences of living with lymphedema among women. Social connectedness and cultural scripts often define a social role for women that transcend physical deformity and disability, while disrupted social connectedness contributes to social isolation, depressive symptoms, and poor health outcomes. Further behavioral research into the contribution of intact social connectedness to resiliency and coping is warranted in order to develop effective interventions for women. Identifying women with disrupted social connectedness and engaging them in behavioral interventions to enhance natural social networks and create new or enhanced social support opportunities may mitigate the negative effects of social disconnectedness and improve quality of life.
\end{abstract}




\section{Introduction}

In the Dominican Republic an estimated $5 \%$ of the population, or approximately 63,000 people, are infected with lymphatic filariasis, a mosquitotransmitted chronic, incurable disease, and an estimated 400,000 people are at risk for infection $(1,2)$. The damage to the lymphatic system this disease causes can result in chronic lymphedema of the leg with episodes of acute bacterial dermatolymphangioadenitis, resulting in fever, swelling of the leg, pain, and nausea. These episodes influence progression of lymphedema and require ongoing coping with disfigurement, adaptation to disability, and pain management by those affected $(3,4)$.

Previous research, predominately with men, has shown that the progression of lymphedema often results in decreased quality of life due to disruption of functional activities of daily living and social roles, loss of employment, threats to body image and self-esteem, and increased psychological distress (5-8). Medical interventions are limited to self-care and management of symptoms, pain, infections, and acute episodes (9). The few studies on women typically address the impact of disfigurement on a woman's ability to marry, but little is known about the overall impact of lymphedema on social connectedness (10,11).

Social connectedness refers to the relationships that people have with others that results in a sense of belonging, a social identity, support and comfort, a buffer for stressors, and positive influences on coping with psychological and physical problems (12-15). Research has described multiple types of support that contribute to coping (12-15). Emotional support through companionship, social interaction, and positive social comparison may contribute to increased self-esteem, intimacy, a sense of belonging, and reassurance of one's self-worth. Instrumental support involves material aid such as money, transportation, medicine, and housing. Those that provide informational support offer advice, engage the person in problemsolving dialogue, and offer cognitive guidance. Strong family relations, social connectedness, and social support have been shown to have protective effects against morbidity and psychological distress in diseases such as coronary heart disease $(16,17)$, rheumatoid arthritis (18), and cancer (19). Social interactions and support contributing to self-efficacy, have been shown to increase one's sense of personal control over situations and conditions that can influence health (20), Social isolation has prospectively predicted mortality and serious morbidity in individuals with established diseases $(16,21)$.

Despite the significant burden of disease found among women living in filariasis-endemic areas of the Dominican Republic, researchers have not described the role of social connectedness and perceived social support in this chronic illness. The objectives of this report are to describe factors associated with intact or disrupted social connectedness among women with lymphedema and the impact of disrupted social connectedness on quality of life. 


\section{Methods}

\subsection{Study area and recruitment}

The study area comprised 10 filariasis-endemic municipalities in the Dominican Republic identified by the Centro Para el Control de Enfermedades Tropicales (CENCET) (2). These areas included densely populated urban neighborhoods (barrios) as well as the rural communities associated with sugarcane production (bateys) (22).

In 2002, CENCET conducted a lymphatic filariasis census in the southern, filarial-endemic region of the. Trained health workers identified anyone with observable lymphedema of the leg. Public health officials referred these women to the Instituto Dermatológico de Cirugia y Piel IDCP in the coastal towns of Santo Domingo and Barahona. Dermatologists screened an estimated 70 women at the IDCP, 30 of whom met the criteria of being newly seen by dermatologists for filarial lymphedema of the leg stages 3-7 (Dreyer et al.); not routinely engaging in medically prescribed lymphedema self-management activities; and expressing willingness to be interviewed, audiotaped, and photographed (9). We were interested in the experiences of women who had not received intensive self-management instruction so that we could clearly distinguish impact of the disease. One tape was damaged and one woman declined to participate in the study resulting in 28 women interviewed. We interviewed women in one sitting with the discussions ranging from 2 to 3 hours. Questions were open-ended with probes as needed to gather detail. Examples of interview questions included: Will you describe for me what it is like for you to live with this swollen leg? What problems has this condition caused you in your life? Will you describe for me how has this affected your life with family, friends, and your community? What types of treatment have you sought for your leg?

An additional 28 women were recruited into focus group discussions (FGD) from the clinic scheduling rosters. The scheduling roster included some women who were current clinic patients that had previously received medically prescribed selfmanagement training for care of their leg and some who were new patients. These women with similar characteristics of interviewees met the same recruitment criteria with the exception that they could be practicing lymphedema management activities which offered some contrasting experiences reflecting on the role of the new program in influencing the typical care and treatment of lymphedema among women. The characteristics of this sample of women appeared typical of Dominican women in general attending IDCP clinics. However, the demands of living with lymphedema and the subsequent toll on their occupational opportunities may have resulted in somewhat more challenged economic circumstances. The interviews and FGDs were conducted by a Dominican social scientist and a research assistant translator assisting the primary author BP.

\subsection{Study design}

We gathered qualitative data through 28 in-depth interview; 3 FGDs; field notes; and photographs of women in clinic, community, and home settings. Data collection was facilitated by interview and moderator guides. Questions and probes 
in the guides were pretested on a sample of local women with lymphedema, and corresponded with local dialect, cultural meanings, and levels of understanding of the women. We conducted interviews over a 2-year period allowing for the review, analysis, and identification of emergent themes to help guide subsequent interviews. $(23,24)$ Data saturation, the point at which no new themes were emerging, was achieved at 24 participants. FGDs were conducted to further explore themes that emerged from the interviews. $(25,26)$ For example, we wanted to know more about the role of the church in providing social support so we discussed that concept in the groups to acquire additional examples and explanations from the women. Findings from the focus groups expanded our conclusions to include the negative social experiences related to church attendance as described by a couple of women in the group which was different than the other women we interviewed.

\subsection{Informed consent}

In keeping with institutional and ethical review board protocols of the US Centers for Disease Control and Prevention (CDC) and CENCET, we obtained written informed consent from every woman in the study.

\subsection{Data management and analysis}

Interviews and FGDs were audiotaped, transcribed verbatim, translated into English by a bilingual research assistant, and entered as a Microsoft Word document into ATLAS-ti to facilitate data coding, text searching, and analysis (27). Transcripts were examined for emerging codes, concepts, categories, and themes (24). The primary author and two research assistants derived coding schemes independently on a subset of interviews and then developed consensus on the codes. Independent coding on another subset of interviews was conducted to ensure consistency in implementing the coding strategy. We drew upon Strauss and Corbin's explanation of open coding, axial coding and selective coding for analyzing the narratives and FGD transcripts (23). We used open coding, a word by word analysis to identify, name and categorize events and explanations found in the text. We followed this with axial coding that requires the analyst to relate themes and categories with their subcategories to identify a more complex analysis of causal relationships in the data. Finally, we used selective coding to integrate and validate the emerging relationships and to construct the explanatory model of disrupted social connectedness. The emergent explanatory model of disrupted social connectedness based upon this inductive analytical process rather than an a priori model prior to analysis is described in the results.

\section{Results}

\subsection{Characteristics of women in the study}

The age range of women in our study was 26-80 years with a median age of 50.5 years. Almost three-fourths of women were married or in a free union relationship and more than three-fourths were literate. More than two-thirds had previously worked at income-generating occupations, and although more than half 
were still working outside the home, most felt they were economically poor. Approximately two-thirds of women had relatives who had lymphedema. Almost half of women had lymphedema in both legs, the others in one leg only. Two-thirds of the women presented with more severe lymphedema, stages 4-6.

\subsection{Generating an explanatory model}

The goal of grounded theory is to generate, inductively, an explanatory model of a phenomenon of interest using empirical data that emerges from qualitative narratives of study (23). Through axial and selective coding, the researcher develops a relational taxonomy of a core category of study. The taxonomy includes existing preconditions, the context in which the core category is embedded, the strategies of action that are used to cope, manage, or influence the contextual categories along with the intervening conditions that moderate the strategies and resulting consequences of taking the described actions, and the phenomena associated with outcomes. We used this analytic process to develop a grounded theory of disrupted social connectedness. The four major categories that emerged during analysis included: the chronic and progressive nature of lymphedema; the role of cultural scripts in defining social identity; social support; and aging.

We constructed a visual representation of the explanatory model of disrupted social connectedness, as shown in Figure 5.1. The chronic and progressive nature of disease, cultural scripts representing social norms and cultural values, social support, and aging displayed at the top of the model provide the context for disrupted social connectedness. Illustrative themes and processes elaborated on within each contextual category resulted in exhaustive attempts of seeking a cure and treatment; impaired social identity, loss of social roles, disrupted social support, and insensitive social interactions; the need for instrumental, emotional, and informational support, and the impact of negative social interactions; and redefined self, fear, uncertainty. Actions and strategies used to influence the themes and processes are depicted in the middle of the model. Categories were also influenced by intervening conditions depicted at the left of the model. The relation of specific negative or uncontrollable properties and dimensions of the intervening conditions with less effective strategies, in the middle of the model, resulted in consequences contributing to disrupted social connectedness noted at the bottom of the model. Themes and processes in the contextual background resulted in adaptive coping responses that evolve, change, and become strained over time as lymphedema progresses and women age, creating situations where adhering to cultural scripts is not feasible, social support is stressed, with all problems compounded by aging. The dynamics of these interactions are complex, resulting in women feeling socially disconnected despite some positive intervening conditions, functional social support, and beneficial coping strategies. The outcomes of disrupted social connectedness that emerged from our data included disrupted social support, poor health outcomes, loss of sense of self, psychological distress, depressive symptoms, and social isolation and exclusion. 


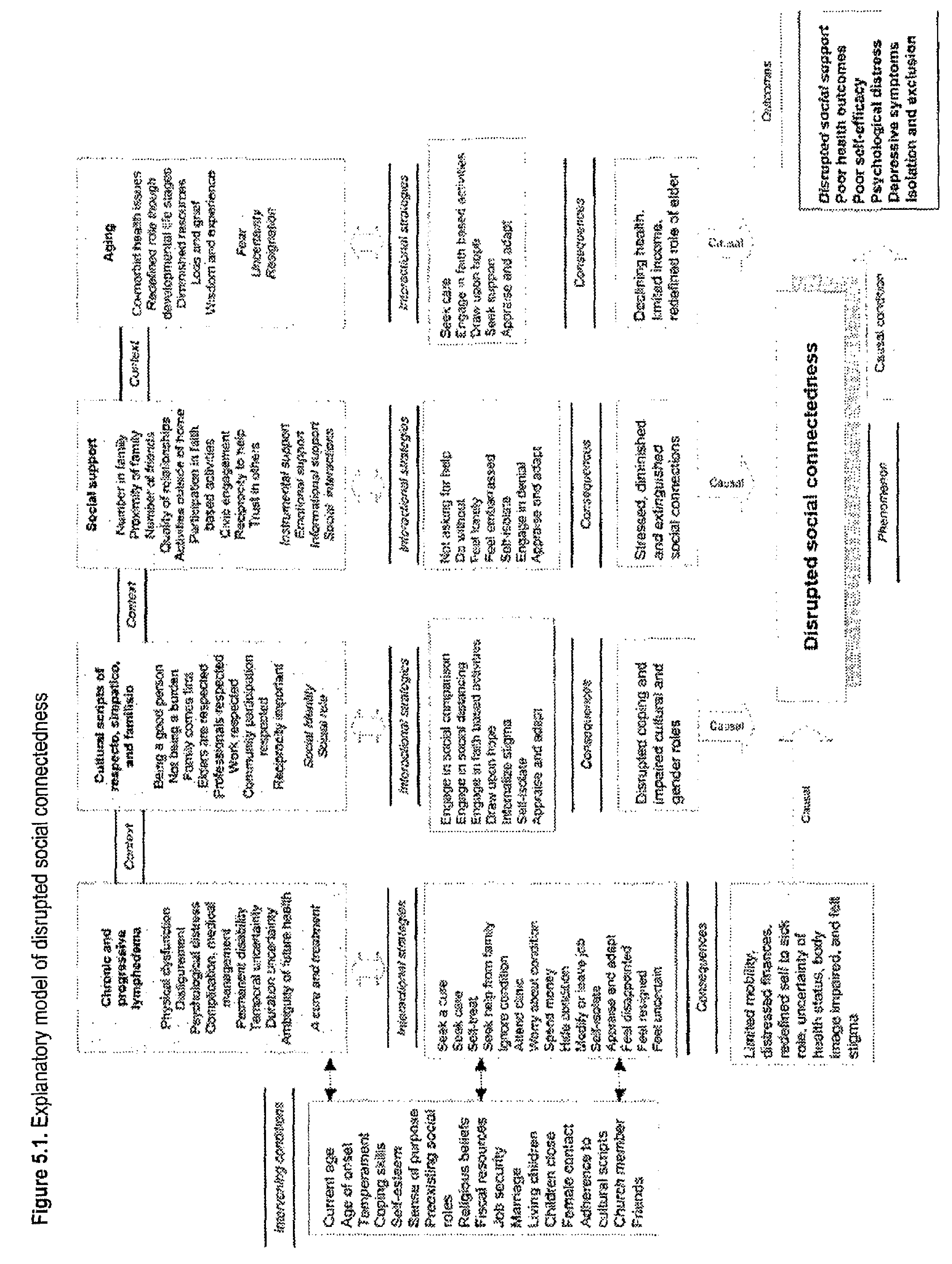




\subsection{Disrupted social connectedness}

Social connectedness refers to the relationships that people have with others that give them a sense of belonging and a social identity. Women in our study described the impact of lymphedema in altering their relatedness to people. Four contextual categories emerged from the data that influenced disrupted social connectedness; they are discussed in the following section.

\subsection{Chronic and progressive lymphedema}

Women in our study described living with lymphedema as intruding, redefining, and engulfing their lives, and creating an uncertain future. A woman said with great despair, "This leg commands all of my life!" Chronicity and the potential for distress related to acute episodes influenced women's appraisal and coping processes. Women used problem-focused coping strategies such as seeking advice, medical and traditional care; self-treating with home remedies or engaging in prescribed wound care; seeking support from others; and covering their leg during social interactions. Women described emotional responses of worry, disappointment, despair, loss of self, impaired body image, and sadness when attempting to cope with pain, disfigurement, loss, and grief towards their condition. Emotion-focused coping strategies were used to regulate emotional responses through cognitive processes such as social distancing, positive downward comparisons, humor, denial, prayer, and avoidance. Failure of these coping processes would often lead to depression, pessimism, and resignation.

The intervening conditions of current age, age of onset, temperament, coping skills, self-esteem, sense of purpose, preexisting social roles, religious beliefs, financial resources, marital status, whether one has children, female contacts, adherence to cultural scripts, and number of friends, as shown in Figure 5.1, interacted both negatively and positively on social connectedness depending upon the relation of categories with intervening conditions and the efficacy of the interactional strategies. Women described suffering when they failed to find concordance with whom they had been and who they were becoming as they had to redefine themselves relative to lymphedema.

Stage of lymphedema was a significant intervening condition whereby women who had more severe lymphedema having often exhausted personal and family resources, interpersonal reciprocity, and emotional ties in attempts to seek a cure. They reported that family members had increasingly less available time to assist them. Women with moderate to severe lymphedema tried to influence their social interactions by hiding their disfigured leg. One woman described her situation by saying, "People looked at my leg and didn't see me. Now my pants cover up my leg and they only see nice legs."

Age of lymphedema onset was important, with women developing lymphedema later in life often appearing less affected due to their better defined social roles and position of status in the church or community. Women described their social connectedness as contributing to a sense of purpose, enhancing selfesteem, and enabling transcendence over disability and disfigurement. Women whose lymphedema began when young, interrupting schooling and work, often 
assumed a sick role that impeded social integration, undermining their social identity, sense of purpose, and self-esteem. The disruption of schooling and work related to early age of onset of lymphedema created a much more influential sick role that impeded social connections.

\subsection{Cultural scripts}

With the progression of lymphedema, women were unable to fulfill expected cultural roles, described as scripts, that are, commonly held assumptions about social interactions, norms, and values by which women assess their own actions $(28,29)$ As a consequence, women experienced disrupted disease management and coping, impaired cultural and gender roles, further eroding social connectedness. Women described the norm of living an interdependent existence and placed great value on social connectedness. This interdependence helped to construct a woman's sense of self with important features of public roles, statuses, and relationships; and was an important contextual category that emerged from the narratives. In this context it was important for women to belong, fit in, promote collective goals, know their place, and intuit what's needed and wanted. The ability to maintain harmony, adjust to a given situation, restrain one's emotions, and put others before self was described as desirable and honored. It was apparent from the narratives that all women were greatly influenced by the concept of familismo, a Latin value of family identification and commitment focusing on unity, loyalty, and solidarity within the nuclear and extended family $(30,31)$. Intact families and strong familismo appeared to support positive perceptions of social connectedness among women with lymphedema while disrupted family connections, poor quality familial relationships, and a woman's feelings of inadequacy to contribute to her family resulted in a sense of social disconnectedness.

The women emphasized the importance of avoiding interpersonal conflict, promoting congenial social interactions, and not being a burden emerged from the narratives of the women interviewed. They attributed receiving positive social support and connectedness to being viewed as a helpful, respectful, and good person with agreeable relationships in their community, the cultural script of simpatia (31). An older woman with moderate lymphedema said, "I have tried to have a good, clean relationship with people. It's very important to not complain. The neighbors worry about me because I have been a good person."

Deference on the basis of age, gender, social status, and economic factors, respecto, was alluded to in the narratives (31). Some women described having limited social support because they were unable to work which could be construed as being lazy. A woman said, "I have no support from any of my neighbors or anyone out there (in the community). You have to work to be respected. I can't work anymore with this leg. If you don't work you are not respected and don't get any help." Women were often hesitant to question indigenous healers, older persons offering suggestions for home remedies, and health care providers even when they were displeased with their medial advice and treatment due to their deference to the person's age or social status. 


\subsection{Social support}

Another contextual category that emerged was social support in which a person's family, friends, and associates constitute important assets that can be called on in a crisis, enjoyed for their own sake, and leveraged for material gain. $(12,13)$ Married women in this study relied heavily on their husbands and described family as their primary source of instrumental support including money, food, medicine, and transportation. Female family members were particularly valued for the emotional support they provided. A woman with severe lymphedema said, "My daughters are a big help. They talk a lot with me and give me advice. Women are more understanding." Some family members provided information, encouraged problem solving, and referred women to traditional healers and medical care. Women often described themselves as being a burden to their families and provided examples of not asking for help. One woman said, "I don't go to weddings or birthdays, because with this leg I would just be in the way. I stay home so as not to be a bother to my family."

The church was a recognized source of instrumental and emotional social support. An older woman said, "If I have an expensive prescription, I take it to the church and they buy it. My false teeth got broken, and they gave me new ones and I didn't pay for them." While some women described the positive emotional support that faith and prayer played in their lives, others described experiences of enacted stigma within their religious community.

Women who described having more family support expressed positive attitudes about life in general, described more variety in their coping strategies, and expressed positive self-esteem and often overcame social stigma. Women who described limited family support appeared to be older, without a spouse due to death or abandonment, and without children close by and were often influenced by social stigma. Some family members engaged in discriminatory behaviors due to fear of contagion. A woman with limited family support said, "My son told me he was afraid of my nasty leg. He doesn't believe that you cannot catch this disease. He doesn't come around me because he is afraid." Women socially isolated themselves due to perceived and felt stigma. A woman with bilateral lymphedema said, "I feel sad. I don't go out into the street unless it's really necessary. I don't like people looking at my legs. I don't see my friends anymore. I am always locked up."

\subsection{Aging}

The final emergent contextual category was aging. Women related growing older and associated morbidities to the need to redefine self through the developmental life cycle, adjust to changing gender roles, and engage in primary and secondary health appraisals and adaptation. A woman feeling alone said, "Of course this condition has affected my relationships! I know that this (lymphedema of the leg) is the reason my husband abandoned me. I am old and have no family. I am nothing." For some women, the stress of repeated failure to find a cure and restrictions from immobility and disability produced feelings of hopelessness and ultimately disrupted social connectedness. 


\section{Discussion and conclusion}

\subsection{Discussion}

The concept of disrupted social connectedness was derived from women's reports on the usefulness and breakdown of their social relations. Women described in-depth the negative impact of filarial lymphedema on their social connectedness and overall quality of life in multiple domains. The richness of their narratives allowed us to create a much more detailed picture of the effects of lymphedema on social connectedness than has been previous reported. The interactions of the emergent categories from the narratives are complex and moderated by a variety of individual and societal factors similar in part to those seen in other chronic, progressive, and debilitating conditions such as rheumatoid arthritis, diabetes, chronic headache, psoriasis, and multiple sclerosis $(32-37)$.

The value of belonging and the subsequent distress and loss of becoming socially disconnected are congruent with the research of Baumeister and Leary on the human need for interpersonal attachment and Cohen on the relation between health and social relationships $(13,38)$. Our findings, similar to the work of Charmaz, highlight the complexity of the life and disease management challenges faced by chronically ill persons who lead restricted lives, experience a discredited sense of self, and exist in social isolation (39). Similar to other studies on social support, support from family and friends, particularly other females, and the church were particularly valued (40-42). Chronic lymphedema, aging, and disability, interacting with other conditions such as diabetes, hypertension, and heart disease, often diminished social interactions, elicited exclusionary behaviors, decreased sense of self worth, and appeared to contribute to the emergence of depressive symptoms among women which decreased their quality of life $(43-45)$.

The women we interviewed were from filariasis-endemic, peri-urban and urban communities in the south coastal region of the Dominican Republic. Caution is suggested when considering these findings in relation to women in more rural areas with less education and fewer resources. The findings from this qualitative study conducted among a fairly small number of women are not generalizable to broader global populations of women with this condition. Social desirability bias may have influenced the responses of some women. The primary author depended upon an experienced Dominican social scientist and research assistant for expressing and interpreting the nuances of dialect during interpersonal exchanges with women in order to be true to interviewing, translation, and data analysis but there is always something lost during translation. Qualitative research is inductive and interpretative thus the analysis may be biased by the experience of the researchers and their conceptual understanding of the culture of participants enrolled in the study.

\subsection{Conclusions}

The explanatory model of disrupted social connectedness details the primary processes that cause social disconnectedness including disease progression, women's failed attempts to adhere to cultural scripts, overtaxed social support resources, 
strained social interactions, damaged social roles in the community, and consequences of aging. The outcomes of disrupted social connectedness that emerged from our data included disrupted social support, poor health outcomes, loss of sense of self, psychological distress, depressive symptoms, and social isolation and exclusion. The condition studied in this research presents extraordinary challenges in terms of being endemic in low resource areas and oftentimes causing extreme disfigurement. However, the constructed explanatory model may offer a way of looking at the impact of other chronic diseases that share either or both the characteristics of occurring in developing country situations or presenting progressive insults to physical health.

Findings from our qualitative research suggest the need for quantitative studies among women with lymphedema to better understand the distribution of the problems expressed by women, the generalizability of our findings, and variation of issues that were noted specifically as related to age and stage of lymphedema. If such research, confirmed the findings in this study, and further, suggested a substantial prevalence of disrupted social disconnectedness, the development, implementation, and evaluation of appropriate behavioural interventions to ameliorate social isolation resulting from disrupted social connectedness would be appropriate.

\subsection{Practice implications}

If sufficient numbers of women with lymphedema experience disrupted social disconnectedness morbidity control programs may need to consider interventions with specific objectives to help women enhance or maintain existing social connections and natural social networks, and create replacements for impoverished or extinguished social connections and networks $(12,46)$. Prevention of disrupted social connectedness could begin with the early medical identification and treatment of lymphatic filariasis. Health education campaigns through radio, television, and printed materials using testimonials, roles model stories, and photo novellas to educate the public could increase knowledge of the disease and encourage screening, treatment, and management activities to forestall lymphedema progression, particularly in areas where stigma is high.

Interventions to improve social connectedness that work within existing natural social networks appear to provide more positive associations of health improvement than those constructed from or provided by strangers $(12,46)$. Strategies to intervene with natural networks include education among the women with lymphedema and their network members to improve communication, social, and relationship skills, remove structural barriers to interacting, and build stronger social ties $(12,47)$. Examples from previous behavioral research has shown that family support groups, visitation programs, and the development of new social networks for family members can strengthen natural helper networks (47-55). For the most disabled women, socially isolated, and without natural social networks, interventions replacing impoverished or extinguished networks may be necessary. These interventions may be in the form of facility or community-based women's groups or health-related support groups for lymphedema education. Further research will assist in determining the best interventions for women with this debilitating condition. 


\section{References}

1. US Department of Health and Human Services. Lymphatic filariasis in the Americas: an epidemiologic history. Atlanta, GA: Centers for Disease Control and Prevention; 2002.

2. Emory University Lymphatic Filariasis Support Center. Lymphatic filariasis in the Americas. 2005. Available at: http://www.sph.emory.edu/lfsc/americas/americas.html. Accessed December 2, 2006.

3. Addiss DG, Louis-Charles J, Wendt JM. Epidemiology of "acute attacks" among patients in a treatment program for filariasis-associated lymphedema of the leg, Leogane, Haiti. Abstract 415. J Am Soc of Trop Med Hyg. 1999; 61(Suppl 3).320.

4. Gasarasi DB, Premji ZG, Mujinja PGM, Mpembeni R. Acute adenolymphangitis due to bancroftian filariasis in Rufiji district, south east Tanzania. Act Trop. 2000; 75:19-28.

5. Ahorlu CK, Dunyo SK, Koram KA, Nkrumah FK, Aagaard-Hansen J, Simonsen PE. Lymphatic filariasis related perceptions and practices on the coast of Ghana. Implication for prevention and control. Acta Trop. 1999; 73:251-64.

6. Ramaiah KD, Guyatt H, Ramu K, Vanamail P, Pani SP, Das PK. Treatment costs and loss of work time to individuals with chronic lymphatic filariasis in rural communities in south India. Trop Med Int Health. 1999; 4:19-25.

7. Suma TK, Shenoy RK, Kumaraswami V. A qualitative study of the perceptions, practices and socio-psychological suffering related to chronic brugian filariasis in Kerala, southern India. Ann Trop Med Parasitol. 2003; 97:839-45.

8. Krishna Kumari A, Harichandrakumar KT, Das LK, Krishnamoorthy K. Physical and psychosocial burden due to lymphatic filariasis as perceived by patients and medical experts. Trop Med Int Health. 2005; 10:567-73.

9. Dreyer G, Addiss D, Dreyer P, Noroes J. Basic lymphoedema management. Treatment and prevention of problems associated with lymphatic filariasis. Hollis, NH: Hollis Publishing Company; 2002.

10. Bandyopadhyay L. Lymphatic filariasis and the women of India. Soc Sci Med. 1996; 42:1401-10.

11. Coreil J, Mayard G, Louis-Charles J, Addiss D. Filarial elephantiasis among Haitian women. Social context and behavioural factors in treatment. Trop Med Int Health. 1998; 3:467-73.

12. Cohen S. Social relationships and health. American Psychologist. 2004; 59:676-84.

13. Cohen S, Gottlieb BH, Underwood LG. Social relationships and health. In: Cohen S, Underwood LG, Gottlieb BH, editors. Social support measurement and intervention: a guide for health and social scientists. Oxford, UK: Oxford University Press; 200o. p. 325.

14. Cohen S, Willis TA. Stress, social support, and the buffering hypothesis. Psychol Bull. $1985 ; 98: 310-57$.

15. Willis TA, Shinar O. Measuring perceived and received social support. In: Cohen S, Underwood LG, Gottlieb BH, editors. Social support measurement and intervention: a guide for health and social scientists. Oxford, UK: Oxford University Press; 2000. p. 86135 .

16. Brummett BH, Barefoot JC, Siegler IC, Clapp-Channing NE, Lytle BL, Bosworth HB, et al. Characteristics of socially isolated patients with coronary artery disease who are at elevated risk for mortality. Psychosom Med. 2001;63:267-72.

17. Kulik JA, Mahler A, Heika I. Emotional support as a moderator of adjustment and compliance after coronary artery bypass surgery. A longitudinal study. J Behav Med. $1993 ; 16: 45-63$.

18. Savelkoul M, Post MW, de Witte LP, van den Borne HB. Social support, coping and subjective well-being in patients with rheumatic diseases. Patient Educ Couns. 2000;39:205-18.

19. Holland $\mathrm{KD}$, Holahan $\mathrm{CK}$. The relation of social support and coping to positive adaptation to breast cancer. Psychol Health. 2003;18:15-9. 
20. Taylor SE, Klein LC, Gruenewald TL, Gurung RA, Fernandez-Taylor S. Affiliation, social support, and biobehavioral responses to stress. In: Suls J, Wallston KA, editors. Social psychological foundations of health and illness. Oxford, UK: Blackwell Publishing; 2003. p. 314-31.

21. Connell CM, Davis WK, Gallant MP, Sharpe PA. Impact of social support, social cognitive variables, and perceived threat on depression among adults with diabetes. Health Psychol. 1994;13:263-73.

22. Vincent AL, Gonzalvo A, Cowell BC, Nayar JK, Uribe L. A survey of bancroftian filariasis in the Dominican Republic. J Parasitol. 1987;73:839-40.

23. Strauss AL, Corbin J. Qualitative research and evaluation methods. Thousand Oaks, CA: Sage Publications; 1998.

24. Charmaz K. Premises, principles, and practices in qualitative research. Revisiting the foundations. Qual Health Res. 2004;14:976-93.

25. Loeb S, Penrod J, Hupcey J. Focus groups and older adults. Tactics for success. J Gerontol Nurs. 2006;32:32-8.

26. Madiz E. Focus groups in feminist research. In: Denzin NK, Lincoln LS, et al. Handbook of qualitative research. Thousand Oaks, CA: Sage Publications; 2000:835-50.

27. Muhr T. ATIAS-ti version 4.2 [computer software]. Berlin: Scientific Software; 1997.

28. CYFERnet. Working with Latino parents/families. Available at: http://www. cyfernet.org/parent/latinofam.html. Accessed December 2, 2006.

29. Marín G, Marin BvO. Research with Hispanic populations. In: Applied Social Research Methods Series, vol, 23. London: Sage Publications; 1991.

30. Flores G. Culture and the patient-physician relationship. Achieving cultural competency in health care. J Ped. 2000;136:14-23.

31. National Center for Mental Health Promotion and Youth Violence Prevention. Meeting the needs of Latino youth: part II: resilience. Available at: http://www.promoteprevent. org/documents/prevention_brief_latino_youth2.pdf. Accessed December 2, 2006.

32. Krol B, Sanderman R, Suurmeijer T, Doeglas D, van Rijswijk M, van Leeuwen M. Medical, physical and psychological status related to early rheumatoid arthritis. Clin Rheumatol. 1995;14:143-50 .

33. Weijman I, Ros WJ, Rutten GE, Schaufeli WB, Schabracq MJ, Winnubst JA. The role of work-related and personal factors in diabetes self-management. Patient Educ Couns. 2005;59:87-96.

34. Tenhunen K, Elnader J. A qualitative analysis of psychological processes mediating quality of life impairments in chronic daily headache. J Health Psy. 2005;10:397-407.

35. Bharath S, Raghuram R, Subbakrishna DK, Shamasundar C. Psychiatric morbidity in leprosy and psoriasis-a comparative study. Indian J Dermatol. 1997;69:341-6.

36. Gordon P. The meaning of disability. How women with chronic illness view their experiences. J Rehab. 1998;July-September.

37. Vardy D, Besser A, Amir M, Gesthalter B, Biton A, Buskila D. Experiences of stigmatization play a role in mediating the impact of disease severity on quality of life in psoriasis patients. Br J Dermatol. 2002;147:736-42.

38. Baumeister RF, Leary MR. The need to belong. Desire for interpersonal attachments as a fundamental human motivation. Psych Bull. 1995;117:497-529.

39. Charmaz $\mathrm{K}$. Loss of self: $\mathrm{s}$ fundamental form of suffering in the chronically ill. Sociol Health Illn. 1983:5:168-95.

40. Taylor SE, Klein LC, Lewis BP, Gruenewald TL, Gurung RA, Updegraff JA. Biobehavioral responses to stress in females: tend-and-befriend, not fight-or-flight. Psy Rev. 2000;107:411-29.

41. Ellison CG, Levin JS. The religion-health connection: evidence, theory, and future directions. Health Educ Behav. 1998;25:700-20.

42. Chatters LM, Levin JS, Ellison CG. Public health and health education in faith communities. Health Educ Behav. 1998;25:689-99.

43. Lazarus RS, DeLongis A. Psychological stress and coping in aging. Am Psy. 1983;38:245-54. 
44. Loeb SJ, Penrod J, Falkenstern S, Gueldner SH, Poon LW. Supporting older adults living with multiple chronic conditions. West J Nurs Res. 2003;25:8-23.

45. Ebrahim S. Aging, health and society. Inter J Epi. 2002;31:715-8.

46. Cutrona $\mathrm{CE}$, Cole, V. Optimizing support in the natural network. In: Cohen $S$, Underwood LG, Gottlieb BH, editors. Social support measurement and intervention: a guide for health and social scientists. Oxford, UK: Oxford University Press; 2000:278308.

47. Coreil J, Mayard G, Addiss D. Support groups for women with lymphatic filariasis in Haiti. Geneva: World Health Organization/TDR; 2003.

48. Gottlieb B. Selecting and planning support interventions. In: In: Cohen S, Underwood LG, Gottlieb BH, editors. Social support measurement and intervention: a guide for health and social scientists. Oxford, UK: Oxford University Press; 2000:195-220.

49. Given BA, Given CW, Kozachik S. Family support in advanced cancer. CA Cancer J Clin. 2001;51:213-31.

50. Stewart MJ, Doble S, Hart G, Langille L, MacPherson K. Peer visitor support for family caregivers of seniors with stroke. Can J Nurs Res. 1998;30:87-117.

51. Gonzalez S, Steinglass P, Reiss D. Putting the illness in its place: discussion groups for families with chronic medical illnesses. Family Process. 1989;28:69-87.

52. Helgeson V, Gottlieb, B. Support groups. In: Cohen S, Underwood LG, Gottlieb BH, editors. Social support measurement and intervention: a guide for health and social scientists. Oxford, UK: Oxford University Press; 2000:195-220.

53. Demissie M, Getahun H, Lindtjorn B. Community tuberculosis care through 'TB clubs' in rural North Ethiopia. Soc Sci Med. 2003;56:2009-18.

54. Jeannine C, Mayard G. Indigenization of illness support groups in Haiti. Human Organization. 2006;65:128-39.

55. Eckenrode J, Hamilton S. One-to-one interventions. In: Cohen S, Underwood LG, Gottlieb BH, editors. Social support measurement and intervention: a guide for health and social scientists. Oxford, UK: Oxford University Press; 2000. p. 246-77. 


\section{Chapter 6}

\section{Health-related Stigma \\ among Women with Lymphatic Filariasis \\ from the Dominican Republic and Ghana}

Bobbie Person, L. Kay Bartholomew, Margaret Gyapong, David Addiss, and Bart van den Borne

Manuscript in Press: Health-related stigma among women with lymphatic filariasis from the Dominican Republic and Ghana. Social Science and Medicine. 2008. 


\begin{abstract}
People fearful of being stigmatized by a health-related condition often do not embrace prevention behaviors or seek needed medical help. They may adhere poorly to treatment regimes for disease and abruptly terminate much needed treatment. Globally, more than one billion people live at risk for infection, and 120 millionmostly poor women-suffer consequences of lymphatic filariasis that include stigmatizing lymphedema or elephantiasis of the leg. Few studies address in-depth the affect of societal and personal stigmatization of this disease on women. We investigated how women with lymphedema from two different cultures experience stigma and its consequences. Our qualitative data were collected from 56 Dominican women and 48 Ghanaian women with lymphedema. A lymphedema-related stigma framework was developed from constructs derived from the literature and emergent themes from the data.

Women described a spectrum of enacted, perceived, and internalized stigma experiences. Some antecedents, consequences, coping strategies, and outcomes of these experiences varied across cultures. Poverty, poor access to health care resources, limited education, and diminished social support challenged the coping strategies of many women and exacerbated negative consequences of lymphedemarelated stigma. We suggest characteristics of interventions to reduce stigma, improve women's coping strategies, and affect the social processes that allow stigma to occur.
\end{abstract}




\section{Introduction}

In early research on stigma, Goffman (1) describes stigma as a damaging social label, or attribute, that taints or discredits the person, influences social interactions, and jeopardizes opportunities for social inclusion. More recently, social scientists have elaborated the roles of labeling, stereotyping, cognition, and differential power and status in stigma $(2-5)$. They have also described social control (6), social structural issues, social processes $(5,7,8)$, culture, and moral experiences of those stigmatized $(9,10)$. Health-related stigma, as defined by Weis et al. (11), "... is typically a social process, experienced or anticipated, characterized by exclusion, rejection, blame, or devaluation that result from experience, perception, or reasonable anticipation of an adverse social judgment about a person or group. This judgment is based on an enduring feature of identity conferred by a health problem or health-related condition, and the judgment is in some essential way medically unwarranted." People fearful of being stigmatized by others may act in ways that undermine coping with illness or exacerbate the disease processes. For example, they may not practice healthy behaviors, seek medical help when needed, or adhere to treatment regimens. They may even terminate treatment prematurely $(12,13)$.

In a recent review, van Brakel (7) compared stigma and related constructs and he identified five categories of health-related stigma; the experience of actual discrimination (enacted stigma); attitudes toward affected people; perceived or felt stigma; self or internalized stigma; and discriminatory practices in (health) services. For this paper, we drew upon the three constructs of enacted, perceived, and internalized stigma to assess stigma as experienced by women with lymphedema. Enacted stigma is characterized as prejudicial attitudes or discriminatory acts by individuals who deny others access to information, health services, social activities, or needed support. Perceived stigma occurs when someone is aware of other peoples' negative attitudes or practices related to their particular condition and anticipates negative social consequences from social interactions. In internalized stigma, ill persons impose stigmatization processes upon themselves and suffer consequences of distressing feelings such as shame or guilt and behaviors such as withdrawal and self-isolation. This paper presents the experiences of women with lymphatic filariasis, a mosquito-transmitted disease caused by the parasite Wuchereria bancrofti often resulting in physically disfiguring and debilitating lymphedema of the leg (14-20). In more than 80 countries, lymphatic filariasis is a major public health problem placing more than one billion people at risk for infection; 120 million individuals, many of them poor women, suffer the consequences of the disease (21). In this paper, we integrated findings that emerged from qualitative research with women who have lymphedema, from two countries, with the concepts of enacted, perceived, and internalized stigma to 1) illustrate stigma processes and 2) suggest evidence- and theory informed programmatic interventions to prevent stigmatization and ameliorate the negative consequences of such experiences. 


\section{Methods}

\subsection{Study area and participants}

This study was conducted in filariasis-endemic areas of the southern coastal region of the Dominican Republic (DR) on the Caribbean island of Hispaniola and in the southern coastal area of the Western and Central regions of Ghana, West Africa (22-24). We recruited women who had lymphedema of the leg, stages 3-7, based on the staging criteria of Dreyer et al. (25). These women were of diverse ages and occupations; did not engage in, were inconsistent with, or had just begun lymphedema self-management activities; and were willing to be interviewed, audiotaped, and photographed (25-27).

Public health officials had recently conducted a combined health education campaign and mass drug distribution to interrupt disease transmission in filariasis endemic regions of both countries. Clinic staff, Ministry of Health staff, and local communicable disease control officers referred women with visible edema of the leg for screening to determine cause of condition and stage of lymphedema. The first 30 women in the DR and Ghana who met the study criteria were referred for an indepth interview.

Of the women recruited, four did not have filarial lymphedema and two declined to participate; there was one incomplete interview; and one interview tape was damaged. In addition, we recruited 24 Ghanaian women from Ministry of Health clinics for 3 focus group discussions (FGDs) and 28 Dominican women from the Instituto Dermatológico de Cirugia y Piel (IDCP) for 3 FGDs. Women were recruited for FGDs from clinic scheduling rosters and records, which included some women who were current clinic patients practicing medically prescribed self-care of their leg and some who were new patients. This mix of patients offered contrasting experiences on the role of prescribed care in influencing the typical care and treatment of lymphedema among women. Women were recruited during the twoweek period before our visit. Those who met the study criteria and agreed to participate were accepted into the study in each country.

\subsection{Ethical review}

Institutional and ethical review boards from the Centers for Disease Control and Prevention (CDC), the Centro Para el Control de Enfermedades Tropicales (CENCET), and the Ministry of Health for Ghana approved this study. Informed consent was read out loud and then signed with a signature or thumbprint by each participant.

\subsection{Design and data collection}

We gathered qualitative data through 52 in-depth interviews; 6 FGDs of 52 women; field notes; and photographs of women in clinic, community, and home settings.

Photographs were taken to allow the study team to confirm stage of lymphedema in the leg. Data collection was facilitated by interview guides translated by bilingual research assistants into local languages of Spanish in the DR and Fanti 
in Ghana. Questions and probes in the guides were pretested with women with lymphedema in both countries to assure correspondence with local dialects, cultural meanings, and women's understanding. We conducted interviews over 2 years so that our preliminary data analysis could help guide subsequent interviews. The 52 interviews, 2-3 hours long, took place in women's homes, local clinics, or outdoor settings. Data saturation, the point at which no new themes emerge, was achieved after interviewing 24 participants in both countries. FGDs were conducted to corroborate interview data, develop theme interpretation, explore concepts derived from individual interviews for greater details, and identify contradicting information. FGDs were conducted in clinic and community settings. Following each interview and FGD, the first author (BP) taught self management regimens that included demonstrations of leg washing, elevation, and exercise; a gift of local soap; and referral to local health care staff for follow-up.

\subsection{Data management and analysis}

Interviews and FGDs were audiotaped, transcribed verbatim, translated into English by bilingual research assistants, and then entered as a Microsoft Word document into ATLAS-ti to facilitate text searching, data coding, and analysis (28). We used open, axial, and selective coding to analyze the narratives and construct a conceptual framework of lymphedema-related stigma (29). We used open coding, a word-by-word analysis to identify, name, and categorize events and explanations of day-to-day reality of participants $(26,29,30)$. We followed open coding with axial coding, the relating of themes and categories with their subcategories to identify causal relationships in the data. Finally, with selective coding we integrated the emerging relationships and classified emergent categories as enacted, perceived, and internalized stigma $(7,31,32)$.

\section{Findings}

\subsection{Characteristics of women who agreed to in-depth interviews}

Characteristics of Dominican and Ghanaian women in the study are included in Table 6.1. Women from the DR and Ghana were similar in age. However, women interviewed in the DR were more literate, had more severe lymphedema, and were more likely to have a family member with lymphedema. DR women reported occupations such as hairdresser, cook, accountant, teacher, small seller, and professor, whereas Ghanaian women reported occupations such as petty trader, farmer, and fish monger.

Table 6.1. Characteristics of women interviewed

\begin{tabular}{lcc}
\hline Characteristics & Dominican women, $\mathbf{N}=28$ & Ghanaian women, $\mathbf{N}=24$ \\
\hline Median age & 50.5 years & 51.3 years \\
Literate (yes) & $22(78 \%)$ & $5(21 \%)$ \\
Stage 3 lymphedema (moderate) & $10(36 \%)$ & $13(54 \%)$ \\
Stage 4-6 lymphedema (severe) & $18(64 \%)$ & $11(46 \%)$ \\
Married or with a partner & $20(71 \%)$ & $12(50 \%)$ \\
Widowed & $5(18 \%)$ & $6(25 \%)$ \\
Divorced or single & $3(11 \%)$ & $6(25 \%)$ \\
Family member with LF & $19(68 \%)$ & $7(29 \%)$ \\
\hline
\end{tabular}




\subsection{Overview of a conceptual framework of lymphedema-related stigma}

We created a visual representation of the women's stigma-related experiences in a conceptual framework (as shown in Figure 6.1). The three central columns of the framework represent the constructs enacted stigma, perceived stigma, and internalized stigma. Under each construct are the causal factors of stigma, women's experiences of stigma, and stigma management strategies, which together result in personal consequences as reported by the women, shown in the box below the three columns. In the far left are the personal and societal influencing factors that women described. On the far right are the resulting health and psychosocial effects of stigmatization. Although the stigma constructs have some independent and unique characteristics, the processes reported by women overlapped in some circumstances. We describe the causal factors, stigma experiences, and management strategies of each stigma construct. We then discuss the overall personal and societal influences related to stigma and, more generally, the personal consequences of the experiences. Finally, we explore the effects of the stigma experiences.

\subsection{Enacted stigma}

Women reported being teased, shunned, marginalized, gossiped about, and discriminated. Such treatment resulted in labeling, public rejection, forced exclusion from social situations, and differential treatment in educational and health care settings. Women reported that disfiguring lymphedema created fear of contagion and worry about potential burden of care among friends and neighbors. These factors were described as the major causes of enacted stigma. Women in both countries were initially hesitant to describe examples of enacted stigma. Some DR women felt that speaking about these practices would be uncharitable and that the burden they faced was a test from God. Ghanaian women described a cultural practice of not talking about or belittling someone with a disease or disability because to do so would put one at risk of getting the same condition. However, the women acknowledged that people did gossip, and cited examples of enacted stigma as they became more comfortable with the interviewers. 


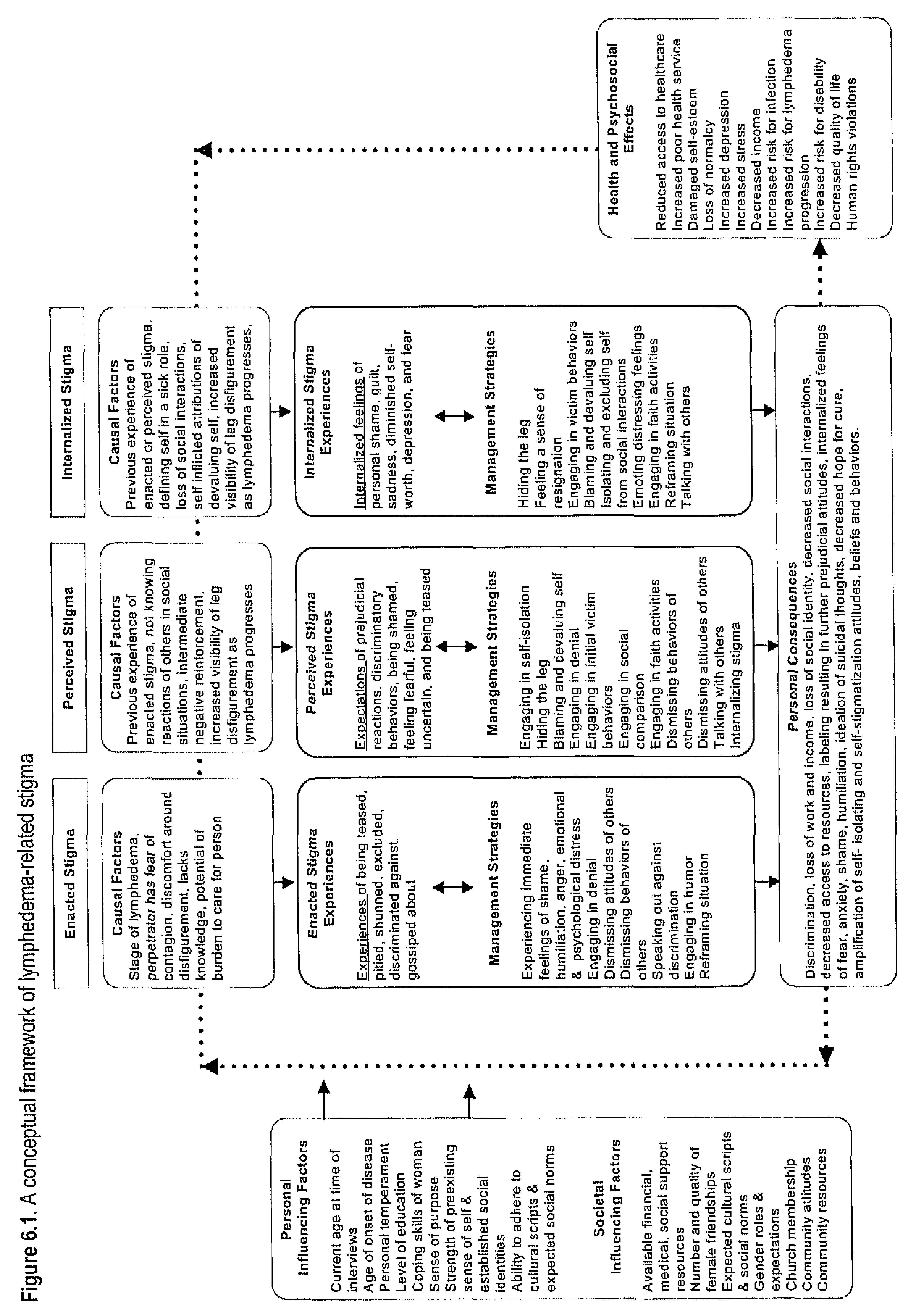


Similar to people with other diseases that result in health-related stigma (33), a Dominican cook said, "My daughter-in-law will not let my son eat at my house. I will cook something for them and she will throw it out. She tells everyone that she is afraid of catching this disease." Friends also engaged in enacted stigma. A Ghanaian woman with severe lymphedema said, "When I was young, I would go out with my friends; but since I had this condition, all my friends have neglected me. They don't call me anymore when they are going somewhere and they don't even greet me when passing by. I feel so shamed by this leg." Institutional stigma exhibited by schools, church members, health care providers, and others often resulted in particularly distressing feelings of despair, loss, hopelessness, and embarrassment. One DR woman described being segregated in a clinic, "The nurse in the waiting room told me that I had to wait outside until I was called to see the doctor because my leg was so disgusting and offended the other patients. Everyone heard her and I was so embarrassed." A young Ghanaian woman described her feelings of social loss, "My foot was so big. It would no longer fit into the white shoes the church choir wore on Sundays, so they told me I could not sing with them anymore. My foot was too disgusting." Several Ghanaian women with early onset lymphedema described their disappointment in not being able to attend school or to attain life goals that education would have afforded. One Ghanaian woman said, "I become very sad because if it had not been for my nasty leg, I would have gone to school and been a teacher. I would have been someone, not just the woman with the leg."

Similar to findings from studies of sufferers of eczema, acne, and other skin disorders $(34,35)$, women from both countries described experiencing distressing insults and teasing about the physical appearance of their legs. Also, in Ghana, children of women with lymphedema were often teased and insulted about their mother's condition. This is commonly known as courtesy stigma, which is enacted stigma imposed upon people associated with a stigmatized individual (1). Ghanaian women described more typically being insulted by adults during moments of anger rather than being teased: "There is a woman who confronts me when she's angry. She says a lot of insults to me. She says bad things about my leg that I can't repeat." Children were more likely than adults to tease and taunt women in the DR: "Sometimes the children in the street make faces and mock me. They make me feel embarrassed and I want to hide," said a Dominican woman.

In societies that place great value on appearance, those who are considered ugly or have a disfigurement are often judged as bad or culpable because of a past indiscretion $(36,37)$. A Ghanaian woman said, "Some people say I did something bad to bring this [big leg] upon myself. I have done nothing!" Similar to findings from other studies, women in both countries described enacted stigma as a contributor to loss of work and income (38). A younger Ghanaian woman said, "People won't shop at my stall in the market any more. People have told me that they are scared of my leg, that it is disgusting. My business is now poor."

Women described feeling pitied by others, but the interpretation of and reaction to pity was different in the two countries. Dominican women described feeling offended by pity, although Ghanaian women construed pity to be helpful or supportive. A Ghanaian woman said, "People pity me because of this leg. They talk to me and if they have money tied at their cloth's end, they give me some to buy food." 
As shown in the middle of the framework, women described negative and positive strategies for managing their enacted stigma experiences. Their appraisal and coping processes for managing their experience of enacted stigma was influenced by the distress associated with the disfigurement of the leg, associated disability, and prejudicial attitudes and discriminatory actions. Women described emotional responses of feeling anger, shame, humiliation, and emotional and psychological distress when coping with gossip, teasing, rejection, exclusion, and differential and poorer treatment in institutional and community settings (39). A Ghanaian woman said, "When I am in the house and I see people coming, I feel afraid and I try to cover my leg with my cloth. I feel sad and embarrassed. I am angry because I am the only one with this condition among these people."

Emotion- and problem-focused coping such as social distancing (from the perpetrator of the stigma), speaking out, use of humor, reframing the situation, and denial of the situation were used to regulate emotional responses (39). Older Dominican and Ghanaian women, particularly those with advanced lymphedema, were more apt to speak out to deflect experiences of enacted stigma or to dismiss the encounters as promoted by ignorant individuals. Older Dominican women also used humor to deflect stigmatization. Younger women in both groups who had early or moderate lymphedema often trying to "pass as normal" would attempt to influence enacted stigma by denial, dismissing such attitudes and actions by describing their condition as something other than filarial lymphedema. When they did acknowledge discriminatory practices, they were more emotionally distressed and described fewer emotion- or problem-focused coping strategies. Ghanaian women often expected, with a sense of resignation, discriminatory actions with little recourse-particularly if it was their husband's or higher status males' or community leaders' engaging in the discriminatory act. One Ghanaian woman described her situation, "If my husband does something bad, I cannot complain or even leave the marriage because of my leg. Who would take me in? Who would help me? He even has a child with another woman. What can I say about it?" Dominican women generally better educated with stronger extended family units and greater financial resources were more apt to describe their negative stigma experiences related to status and power differentials to be associated with institutions such as schools, businesses, and health care facilities.

Women in both countries described no recourse for entrenched institutional and structural stigmatization that lead to job loss, societal marginalization, unequal access to health care, and ostracism by the educational system. Women described significant experiences of enacted stigma and discrimination by health care providers, often discouraging them from seeking care in the future.

\subsection{Perceived stigma}

Perceived stigma experiences included expectations of discrimination, along with fear of and uncertainty about being marginalized, shamed, teased, gossiped about, or shunned. Similar to findings from other stigma studies, there appeared to be a discrepancy between experiences of perceived stigma compared with enacted stigma reported by women (31). Perceived stigma typically occurs when individuals experience negative social interactions causing them to anticipate similar 
interactions in the future (7). These expectations can also occur when people have not previously experienced enacted stigma.

Women in our study often believed that strangers and family and community members would negatively judge, devalue, or verbally belittle them owing to the physical appearance of their leg. Studies of patients with TB, epilepsy, HIV/AIDS, and mental illness show that perceived stigma is often associated with poor selfworth and low self-esteem (4,40-42). Similarly, for some women in this study, perceived stigma also gave rise to feelings of fear, anxiety, loneliness and lowered self-esteem even though they were not able to describe a specific discriminatory act. A Dominican woman with moderate lymphedema said, "I am afraid to go out. I think that people will look at me as if they were looking at something very strange. It has happened before. I stay home now. I don't go out because one doesn't know what strangers will do." Describing similar feelings, a Ghanaian said, "I am afraid to go out because I know people will stare at me because of my leg. Then they will see that this disease has embarrassed me. They will make me sad."

Many Dominican women perceived that their husbands were supportive whereas Ghanaian women, facing greater economic vulnerability, often described a fear that their husbands might take other wives and abandon them if their leg became grossly disfigured, further stigmatizing them as undesirable. Although women anticipated potential discrimination or victimization by others because of their lymphedema, they often felt their leg had not affected their relationships with most family members. Women who anticipated poor treatment from family members attributed potential discrimination to a family member's fear of contagion, disgust at the sores and size of the leg, and potential burden associated with caring for the woman. Due in part to perceived stigma and despite support from family, friends, and church, many Dominican women feel socially disconnected (15). The social support network outside the immediate family of Ghanaian women was distinctly smaller than that of the Dominican women, with the attrition of relationships often attributed to perceived stigma. A Ghanaian woman said, "When I was young, I go to town and play with my friends. But since I had this condition, all my friends have neglected me. They don't call me anymore when they are going somewhere, and they don't even greet me when passing."

Internalized feelings associated with previous experiences of enacted stigma and cultural norms associated with their condition influenced women's appraisal and coping processes for managing perceived stigma. They described feeling fear, anxiety, sadness, and psychological distress when attempting to cope with perceived stigma and with the ever-present anticipation of enacted stigma. Some positive and negative strategies for managing stigma that women described were similar to cognitive and behavioral processes for coping with enacted stigma. In addition to those strategies, women also described using social comparison by identifying others less fortunate than themselves; by social distancing from the possible perpetrator of the stigma; by attributing the situation to the "will of God"; and by further internalizing of stigma. One Ghanaian woman said, "I have the hope that God will heal me-but if not, I have to bear the burden he places upon me for whatever I have done wrong." 


\subsection{Internalized stigma}

Women reported experiences of enacted stigma and feelings of perceived stigma as contributing to their internalized stigma. Internalized stigma was described by women across all stages of lymphedema from both countries as a process of self-labeling with feelings of fear, guilt, shame, sadness, depression, and diminished self-worth. Causal factors of self-stigmatization included women's identification with a sick role, loss of social interactions contributing to self-inflicted attributions of devaluing self, and further distress associated with gross disfigurement of the leg as lymphedema progresses.

Women engaged in similar stigma management strategies as for perceived stigma such as hiding their legs during social interactions; engaging in victim behaviors, isolation, or denial; and blaming and devaluing self. A Dominican woman with only moderate lymphedema said, "Everyone who sees me says something about my leg. It bothers me. It is a shameful thing. I get so embarrassed. I am afraid it will get bigger." Women in Ghana who had fewer resources, lower literacy, less community support, and fewer income-generating options were more likely to hide their legs in an attempt to pass as normal and avoid enacted stigma. Research suggests that trying to conceal a disfiguring condition to avoid the potential for an enacted stigma often increases stress owing to the fear and anxiety of perhaps being found out (43).

Women also engaged in victim behaviors that increased stress rather than inhibiting it. Ghanaian women described greater emotional distress because of lymphedema than Dominican women. After contemplating suicide, a Ghanaian woman said, "You see the pain that I am going through with this leg. I wished God had ended it all by taking my life. I think about that, I do."

As seen in other progressively stigmatizing conditions, when lymphedema progresses, it becomes more difficult to pass as normal and women attempt to hide the condition via clothing or resorting to avoidance, self-isolation, and internalizing stigma $(1,39,43)$. For example, a woman's inability to wear shoes made social interactions difficult in both countries and contributed to low self-esteem, social disconnectedness, and grief for loss of normalcy $(15,39,44)$. "Look, I cannot wear shoes. I can't go out because I can't put my shoes on. I don't want to go out to the street because people stare. My clothes do not fit me, my pants don't fit, and my shoes don't fit. It makes me so depressed! I don't belong in society any more. I am nothing now," said a Dominican woman who had once been a hairdresser. Many women thought less of themselves than before they had this condition. A 46-year-old Ghanaian woman, describing her impaired sense of self said, "Many times I don't go out. I don't want my leg seen. Here I have one good leg, and the other is so bad. My leg is so very fat and ugly. I am no longer a woman. I no longer have a life." Some women who expressed internalized feelings of shame, embarrassment, and diminished sense of self-worth described thoughts of ending their life; women in Ghana described thinking more about this than women in the Dominican Republic. A Ghanaian woman said, "There is a woman with a very big leg that has many sores. I have thought sometimes about taking rat poison if my leg gets like hers. One can not really live with a leg like that." Women, who positively reframed their situation, drew upon the support of others and engaged in faith-based activities to cope with 
their condition and manage stigma experiences. "My church members visit me when they don't see me. Talking with them gives me strength to live with this leg. I remember that God loves me no matter what people think about my leg," said one Ghanaian woman. Some women with more advanced lymphedema, who did not try to hide their legs, expressed a stronger sense of personal self-worth or often described a resigned or "why bother" attitude.

\subsection{Personal and societal influencing factors}

The interaction between stigma experiences and management strategies, along with consequences of these interactions, was influenced by personal and societal factors. Personal influencing factors, shown on the left side of the framework, included age; age at onset; personal temperament; level of education; coping skills; sense of purpose; strength of preexisting sense of self and established social identities; and the ability to adhere to cultural scripts and expected social norms. Societal influences included available financial, educational, medical, and social support resources; number and quality of female relationships; expected cultural scripts and social norms; gender roles and expectations; church membership; community attitudes; and community resource. Negative personal and societal factors can cause stigma management strategies to fail and the consequences are often distressing (15).

\subsection{Personal consequences of enacted, perceived, and internalized stigma}

Cognitive, emotional, and behavioral consequences that emerged from the stigma processes were difficult to attribute to one stigma construct. These consequences included discrimination; loss of work; loss of social identify, decreased social interactions; decreased access to resources; labeling, resulting in further prejudicial attitudes; internalized feelings of shame, anger, or humiliation; psychological and emotional distress; ideation of suicidal thoughts; decreased hope for a cure; amplification of self-loathing; and self-stigmatization (39). For some women, it was the quantity or burden of stigma experiences taxing ineffective management or coping capabilities that created distressing personal consequences. For others, the quality or intensity of even one experience could impair health and psychosocial outcomes.

\subsection{Health and psychosocial effects of stigma}

The confluence of stigma consequences often becomes deep-seated in the women's psyche. Results can include decreased income and psychosocial effects (depression, damaged self-esteem, and loss of normalcy) (17,39). This "confluence of stigma consequences" also results in health effects such as reduced access to health care; poor health services; increased risk for infections; lymphedema progression; and increased risk for disability; lower quality of life, and sometimes human-rights violation. Self-degrading attitudes contribute further to an overall decreased quality of life. "I feel very sad because with this leg I am now a sick, worthless person, and have no life," said one Ghanaian woman. 


\section{Discussion}

There is little information in the literature detailing the effect of lymphatic filariasis on the lives of women. Further, there are few data on quality of life and the experiences of stigma that women with lymphedema endure. While there is increasing interest in the monitoring, measuring, and intervening upon stigma across cultures, there are few cross-cultural studies on stigma. Drawing upon findings from our formative research, we posit that the three stigma constructs transcend the two cultures; have some underlying characteristics that overlap; and amplify the problems that women experience as lymphedema progresses, resources dwindle, and women age.

The constructs of enacted, perceived, and internalized stigma provided a framework for describing a cycle of stigmatization experienced by women in both countries that begins with disfigurement of the leg. This disfigurement prompts prejudicial attitudes and discriminatory behaviors by others. These behaviors often induce internalized shame, guilt, fear, and other degrading feelings. These feelings can result in withdrawal, avoidance, and self-isolation behaviors.

Women in both countries described strategies for managing stigma. The use and effectiveness of these management strategies, both negative and positive, were influenced by personal factors (i.e., age, age at onset, education, fiscal means) and by societal factors (i.e., preexisting social roles, friends and family, and adherence to cultural scripts).

Stigma processes in interaction with management strategies lead to health and psychosocial outcomes similar to those described in studies of people living with leprosy, cutaneous leishmaniasis, Buruli ulcer, and onchocercal skin disease (12,4548). Women in both countries described fear of contagion and personal discomfort related to the smell, sores, and physical disfigurement of their leg as contributing to enacted stigmatization by others. The consequences of enacted stigma redefined the women's occupational roles and resulted in a loss of income, a loss of a social identity, decreased social interactions and access to resources, and labeling with further stigmatization. These enacted stigma experiences often led to perceived and internalized stigma contributing to increased fear, anxiety, depression, and suffering along with amplified self-isolating, self-demeaning, and sick role behaviors, impaired body image, and in some cases suicidal thoughts. As in other studies, the self-degrading feelings that women described were often perceived more deleterious than the actual course of the disease (31).

Yang's work on the moral experience of stigma encourages us to closely examine what is most at stake for women to lose in their everyday living with this condition (10). Ghanaian women reported greater suffering, sensing a loss of purpose in their lives along with diminished social roles, decreased self-esteem, significantly fewer resources, lack of education, and inability to work and care for themselves and their children. Many Ghanaian women felt that their inability to work and contribute to society was their greatest loss. Dominican women were more likely to have a job or sense of purpose, greater financial resources, a better education, a strong connection to the faith community, and support from family members with more resources to share. These women described the loss of their sense of normalcy in appearance and ability to be a social person as their greatest loss. 


\subsection{Stigma reduction interventions}

The consequences of stigma experiences as reported by women in this study suggest that interventions to prevent stigma and to mediate its negative effects (whether experienced, perceived, or internalized) should be a critical component of any lymphedema management effort. The framework presented in this study depicts stigma as a multilevel social process that must be understood within the broader social context of illness, the appropriate cultural framework of the affected audience, and the moral experience of those affected. Interventions based on this ecological framework should address both the woman with lymphedema and the interpersonal, organizational/institutional, community, and governmental structures and processes that cause stigma $(11,32,49)$.

Interventions that target perpetrators of stigma and attempt to shift social norms should address fear of contagion, discomfort with disfigurement, and lack of knowledge on transmission of disease. Interventions designed to reach the general public and perpetrators of stigma could be more efficacious if a mix of print materials, narrative communication strategies, videos, public presentations, mass media, media publicity, role play, classroom exercises, street theater, and even new technologies (mobile phone text messaging) were part of the intervention. Informational interventions aim to provide factual information, dispel myths, and enhance empathy with an affected person by emphasizing that health status is not the only relevant identity feature of a person with a stigmatized condition.

Informational interventions provided through peer education, lectures, pamphlets, or workshops are increasing positive attitudes toward people living with AIDS (50). Lymphedema management programs can offer the public opportunities to know someone with lymphedema through vicarious contacts with affected women (via television or print media), but radio is often the preferred communication channel in developing countries. Messages of hope and the availability of new optimistic treatments can discredit stigmatizing notions and serve as a strong antistigma message (51). Optimistic public education campaigns that describe the benefits of early identification and treatment to prevent or slow the disfiguring and disabling consequences of lymphedema may dispel fear-related stigma.

Additionally, stigma may be eased by educating the family about the disease process and by openly discussing fears and concerns. When informed, family members can counteract the misperceptions and fears of those with whom they interact by providing positive, accurate information about the diagnosis, transmission, treatment, and management of lymphedema. Family support groups can offer psycho-educational and medical information, as well as emotional and problem-solving skills for handling negative social interactions associated with a family member with lymphedema (52).

Interventions for those who experience perceived or internalized stigma should address feelings of fear, shame, depression, self-loathing, embarrassment, psychological distress, and anticipation of discrimination and social exclusion. Interventions that include emotion- and problem-focused coping strategies, psychosocial educational approaches, and resource acquisition could mediate individual effects of stigma. Social and family networks are critical to women with 
lymphedema, and based on attitudes, beliefs, and behaviors, can be both perpetrators and recipients of stigma. Tailored interventions could be beneficial.

Research shows that individuals who have experienced the same problems as those affected with a stigmatizing health condition are considered purveyors of more credible and accurate information than others. Further, empathetic individuals are less likely to promote stigmatizing attitudes and behaviors (1). The shared difficulties of women who have experienced stigma can be the basis for psycho-educational and problem-solving peer support groups in which information and empathy can be conveyed (52-54). Support group interventions for women with lymphedema in one study focused on strengthening resiliency and reducing vulnerability through psycho-educational efforts and activities to increase self-esteem, to provide incomegenerating activities, and to give emotional and social support in dealing with stressful situations (52).

Stigma by health care providers can be challenging to address. As shown in the HIV/AIDS literature, creating a planned and prescribed opportunity for health care providers and others to actually interact with a person who has a stigmatizing condition in such a way that normalizes the person appears to reduce stigma and fear (55). A prescribed socializing contact with a woman with lymphedema could humanize this stigmatizing, disfiguring condition. Individual or small group encounters where women with lymphedema can talk to health care providers and others about themselves, describe the challenges of living with this condition, and dispel myths about the condition could diffuse fear and judgment while encouraging empathy. Continuing education opportunities on the care of women with lymphedema could give health care providers the knowledge to treat the disease appropriately and could provide greater comfort when providing care. In one study, people living with HIV/AIDS helped teach an AIDS course for nursing students. The successful course encouraged student nurses to examine their own stigmatizing attitudes. Through group discussions, they further resolved their dissonance between wanting to provide care and feeling fear that leads to enacted stigma toward patients $(56)$.

\subsection{Study limitations}

Social desirability bias may have influenced the responses of some women. To interpret the nuances of dialect during interpersonal exchanges with women, we depended upon experienced local interviewers, research assistants, and investigators. However, something is always lost during translation. Qualitative research is inductive and based on observations and interpretation. Thus, the analysis may be biased by the experience of the researchers and their conceptual understanding of the culture of participants enrolled in the study.

\section{Conclusion}

The conceptual framework of lymphedema-related stigma details the processes that contribute to women's experiences of stigma-fitting with the concepts of enacted, perceived, and internalized stigma drawn from the literature. Our findings suggest that lymphedema-related stigma is a social, cultural, and moral process that needs to be explored further through qualitative and quantitative 
research to better determine whether the problems expressed by women in our study are generalizable to other women with this condition. If these findings are substantiated, then appropriate behavioral interventions to prevent stigma and to ameliorate the negative effects of stigma are needed. Intervening with these social, cultural, and moral processes requires multifaceted, ecologically tailored interventions for those who experience perceived or internalized stigma; for the perpetrators of prejudicial attitudes and discriminatory behaviors resulting in enacted stigma; and for the social systems that promote or fail to inhibit those processes. Intervening to reduce stigma would likely mean that women would seek care earlier, develop more effective coping strategies for living with this chronic condition, engage in more positive social interactions, have greater access to resources, and have hope for the future. Additionally, research is needed to better understand how some resilient women, in both cultures, were able oppose and overcome the disfigured and discredited sense of self that contributes to the intensity and internalization of stigma experiences. 


\section{References}

1. Goffman E. Stigma: notes on the management of spoiled identity New York: Simon \& Schuster; 1963.

2. Link BG, Phelan JC. Conceptualizing stigma. Annual Review of Sociology. 2001;27:36385.

3. Sartorius, N. Lessons from a 10-year global programme against stigma and discrimination because of an illness. Psychology, Health Medicine. 2006;11(3):383-8.

4. Scambler G, Hopkins A. Being epileptic: coming to terms with stigma. Sociological Health. $1989 ; 8 ; 26-43$.

5. Scambler G. Sociology, social structure and health-related stigma. Psychol Health Med. 2006;11(3):288-95.

6. Dijker AJ, Koomen W. A psychological model of social control and stigmatization: Evolutionary background and practical implications. Psychol Health Med. 2006; 11(3):296-306.

7. van Brakel WH. Measuring health-related stigma-a literature review. Psychol Health Med. 2006;11:307-34.

8. Corrigan PW, Penn DL. Lessons from social psychology on discrediting psychiatric stigma. The American Psychologist. 1999;54(9):765-76.

9. Kleinman A, Benson P. Culture, moral experience and medicine. The Mount Sinai Journal of Medicine. 2006;73(6):834-9.

10. Yang LH, Kleinman A, Link B, Phelan JC, Lee S, Godd B. Culture and stigma: adding moral experience to stigma theory. Soc Sci Med. 2007;11(13).

11. Weiss M, Ramakrishna J, Somma D. Health-related stigma: rethinking concepts and interventions. Psychol Health Med. 2006;11(3):277-87.

12. Das V. Stigma, contagion, defects: issues in the anthropology of public health. In: J.H. University, editor. US NIH Conference on Stigma and Global Health: developing a Research Agenda. Bethesda, MD; 2001.

13. Eastwood SV, Hill PC. A gender-focused qualitative study of barriers to accessing tuberculosis treatment in The Gambia, West Africa. Int J Tuberc Lung Dis. 2004; $8(1): 70-5$

14. Bandyopadhyay L. Lymphatic filariasis and the women of India. Soc Sci Med. 1996; 42:1401-10.

15. Person B, Addiss D, Bartholomew LK, Borne B. Disrupted social connectedness among Dominican women with chronic filarial lymphedema. Patient Education and Counseling. 2007;68(3):279-86.

16. Person B, Addiss DG, Bartholomew LK, Meijer C, Pou V, Borne BV. Health-seeking behaviors and self-care practices of Dominican women with lymphedema of the leg: implications for lymphedema management programs. Filaria J. 2006;5(1):13.

17. Gyapong M, Gyapong JO, Adjei S, Vlassoff C, Weiss M. Filariasis in northern Ghana: some cultural beliefs and practices and their implications for disease control. Soc Sci Med. 1996;43:235-42.

18. Ramaiah KD Kumar KN, Ramu K. Knowledge and beliefs about transmission, prevention and control of lymphatic filariasis in rural areas of south India. Trop Med Int Health. 1996;1(4):433-8.

19. Ramaiah KD, Kumar KNV, Ramu K, Pani SP, Das PK. Functional impairment caused by lymphatic filariasis in rural areas of South India. Trop Med Int Health. 1997;2:832-8.

20. World Health Organization. Lymphatic filariasis: progress of disability prevention activities. Weekly Epidemiological Record. 2004;79(47):417-24.

21. Ottesen EA, Duke BOL, Karam M, Behbehani K. Strategies and tools for the control/elimination of lymphatic filariasis. Bull World Health Organ. 1997;75(6):491503 .

22. Gyapong JO. The relationship between infection and disease in Wuchereria bancrofti infection in Ghana. Trans R Soc Trop Med Hyg. 1998;92:390-2. 
23. Gyapong JO, Adjei S, Sackey SO. Descriptive epidemiology of lymphatic filariasis in Ghana. Trans R Soc Trop Med Hyg. 1996;90(1): 26-30.

24. US Department of Health and Human Services. Lymphatic filariasis in the Americas: an epidemiologic history. Atlanta, GA: Centers for Disease Control and Prevention; 2002.

25. Dreyer G, Addiss D, Dreyer P, Noroes J. Basic lymphedema management: treatment and prevention of problems associated with lymphatic filariasis. Hollis, NH: Hollis; 2002.

26. Charmez K. Constructing grounded theory: a practical guide through qualitative analysis. London: Sage Publications; 2006.

27. Patton M. Purposeful sampling. Qualitative research and evaluation methods. Thousand Oaks: Sage Publishers, Inc.; 2002. p. 230-43.

28. Muhr T. ATLAS-ti version 4.2 [computer software]. Berlin: Scientific Software; 1997.

29. Strauss A, Corbin J. Basics of qualitative research: techniques and procedures for developing grounded theory. Thousand Oaks, CA: Sage Publications; 1998.

30. Pandit NR. The creation of theory: a recent application of the grounded theory method. Qualitative Report. 1996.

31. Jacoby A. Felt versus enacted stigma: a concept revisited. Evidence from a study of people with epilepsy in remission. Soc Sci Med. 1994;38(2):269-74.

32. Somma D, Bond V. International research workshop on health-related stigma and discrimination. Psychol Health Med. 2006;11(3):271-6.

33. Ogden J, Nyblade L. Common at its core: HIV-related stigma. Washington, DC: International Center for Research on Women; 2004.

34. Jowett S, Ryan T. Skin disease and handicap: An analysis of the impact of skin conditions. Soc Sci Med. 1985;20(4):425-9.

35. Kent G. Correlates of perceived stigma in vitiligo. Psychology and Health. 1999;14:24151.

36. Feingold A. Good looking people are not what we think. Psychol Bull. 1992;111:304-41.

37. Umberson D, Hughes MH. The impact of physical attractiveness on achievement and psychological well-being. Social Psychological Quarterly. 1987;60:227-36.

38. Ramaiah KD, Guyatt H, Ramu K, Vanamail P, Pani SP, Das PK. Treatment costs and loss of work time to individuals with chronic lymphatic filariasis in rural communities in south India. Tropical Medicine International Health. 1999;4:19-25.

39. Person B, Addiss D, Bartholomew LK, Meijer C, Pou V, Gonzálvez G, Borne B. Can it be that God does not remember me?: A qualitative study on the psychological distress, suffering and coping of Dominican women with chronic filarial lymphedema and elephantiasis of the leg. Healthcare for Women International. In press.

40. Alonzo AA, Reynolds NR. Stigma, HIV and AIDS: an exploration and elaboration of the illness trajectory surrounding HIV infection and AIDS. Soc Sci Med. 1995;41:303-15.

41. Corrigan PW, Calabrese JD. Strategies for assessing and diminishing self-stigma. In: PW Corrigan, editor. On the stigma of mental illness. Practical strategies for research and social change. Washington, DC: American Psychological Association; 2005.

42. Link BG, Struening EL, Neese-Todd $S$, et al. The consequences of stigma for the selfesteem of people with mental illnesses. Psychiatric Services. 2001;52:1621-6.

43. Porter J, Beauf A, Lerner A, Norlund J. Response to cosmetic disfigurement: Patients with vitiligo. Cutis. 1987;39:493-4

44. Person B, Addiss D, Bartholomew LK, Meijer C, Pou V, Gonzálvez G, et al. A qualitative study of the psychosocial and health consequences associated with lymphedema among women in the Dominican Republic. Acta Tropica. 2007;103(2):90-7.

45. van Brakel WH. Measuring leprosy stigma-a preliminary review of the leprosy literature. International Journal of Leprosy and Other Mycobacterial Diseases. 2003; $71(3): 190-7$

46. Castle S. Rural children's attitudes to people with HIV/AIDS in Mali: the causes of stigma. Culture, Health Sexuality. 2004;6(1):1-18.

47. Stienstra $Y$, van der Graaf WT, Asamoa K, van der Werf 'TS. Beliefs and attitudes toward Buruli ulcer in Ghana. American Journal of Tropical Medicine and Hygiene. 2002; 67(2):207-13. 
48. Yanik M, Gurel MS, Simsek Z, Kati M. The psychological impact of cutaneous leishmaniasis. Clinical and Experimental Dermatology. 2004:29(5):464 -76.

49. Birbeck G. Interventions to reduce epilepsy-associated stigma. Psychol Health Med. 2006;11(3):364-6.

50. Brown L, Macintyre K, Trujillo L. Interventions to reduce HIV/AIDS stigma: What have we learned? AIDS Education and Prevention, 2003;15(1):49-69.

51. del Vecchio P. Commentary: all we are saying is give people with mental illnesses a chance. Psychiatric Serv. 2006;57:646.

52. Coreil J, Mayard G, Addiss D. Support groups for women with lymphatic filariasis in Haiti. Geneva: WHO/TDR; 2003.

53. Demissie M, Getahun H, Lindtjorn B. Community tuberculosis care through 'TB clubs' in rural North Ethiopia. Soc Sci Med. 2003;56:2009-18.

54. Lyon $M E$, Woodward $K$. Nonstigmatizing ways to engage HIV-positive AfricanAmerican teens in mental health and support services: a commentary. J Natl Med Assoc. 2003;95(3):196-200.

55. Herek GM, Capitanio JP. AIDS stigma and contact with persons with AIDS: effects of direct and vicarious contact. J Appl Soc Psychol. 1997;27(1):1-36.

56. Wyness MA, Goldstone I, Trussler T. Outcomes of an undergraduate HIV/AIDS nursing elective: insightful learning to promote quality care. The International Conference on AIDS. Vancouver, British Columbia, Canada; 1996. p. 21 


\section{Chapter 7}

\section{Evidence- and Theory-informed Recommendations for Program Planning to Improve Self-management of Filarial Lymphedema in Women}

Bobbie Person, L. Kay Bartholomew, David Addiss, and Bart van den Borne

To be submitted as: Evidence- and theory-infromed recommendations for program planning to improve self-management of filarial lymphedema in women. 


\begin{abstract}
Health planners in resource-poor countries have to address human behaviors as well as environmental factors when planning for programs to promote early diagnosis, treatment, and participation of women in the ongoing management of lymphedema of the leg and associated psychological distress, often the sequelae of lymphatic filariasis. In this chapter, Intervention Mapping (IM), a framework for systematic health promotion program planning and evaluation, guides the process for developing recommendations for an integrated lymphedema program of self-care for lymphedema along with a component of coping with the psychosocial complications for affected women that can be adapted by resource poor countries. Data were derived from a formal qualitative needs assessment and health problem analysis with women with lymphedema in filariasis endemic areas of Ghana and the Dominican Republic to informal the IM process. Additional information was gathered through informal discussions with family members of women and stakeholders from the communities at-risk and the healthcare and public health sectors. Examples of behavioral self-regulation theories associated with behavioral determinants, intervention methods, and practical strategies to encourage women to consistently engage in the desired behaviors are suggested to address identified needs. Program components to enhance a healthcare provider's ability to diagnose and manage the physical and psychological sequelae of disease and stigma reduction strategies are proposed. The recommendations derived from the evidence and theory-informed examples in this paper may serve as a viable starting point for program planners wanting to enhance their lymphedema management programs for affected women.
\end{abstract}




\section{Background}

Lymphatic filariasis is one of 13 parasitic and bacterial infections considered by public health practitioners as a neglected tropical disease $(1,2)$. Neglected diseases are some of the most ordinary illnesses affecting more than 2.5 billion people that live in poverty worldwide $(1,3)$. Lymphatic filariasis is a mosquito-transmitted parasitic disease caused by roundworms that inhabit the human lymphatic vessels and subcutaneous tissues predisposing the person to lymphatic dysfunction $(4,5)$. More than one billion people are at risk for infection in more than 80 countries and an estimated 44 million people are suffering from chronic symptomatic conditions such as lymphedema, hydrocele, chyluria, and episodic bouts of bacterial dermatolymphangioadenitis $(3,6-8)$. Thirty-two of the thirty-eight least developed countries in the world are endemic for lymphatic filariasis (9).

Episodic bouts of acute dermatolymphangioadenitis, experienced by most with this condition, appear to influence progression of lymphedema and require ongoing coping, social adaptation, and pain management by those affected (10). These episodes are characterized by fever, chills, and painful inflammation and swelling of the limb, often followed by exfoliation of the skin (10-16). Previous research, predominately with men, has shown that the progression of lymphedema often results in decreased quality of life due to disruption of functional activities of daily living and social roles, loss of employment and income, threats to body image and self-esteem, and increased psychological distress (17-24). Women in resourcepoor countries oftentimes face an uncertain future when the confluence of chronic and acute stressors associated with lymphedema is exacerbated by aging, disrupted social connectedness, and decreasing resources (25-29).

In 1998, the Global Program to Eliminate Lymphatic Filariasis (GPELF) was established by the World Health Organization with a goal of eradication of the disease by the year 2020 (30). The GPELF was based upon the two pillars of interruption of transmission of disease and the implementation of morbidity control for affected individuals (31). While many countries are attempting to initiate both strategies the bulk of resources of the GPELF are focused on interrupting transmission. Even if disease transmission was successfully interrupted there would be millions of people left to deal with the sequelae of disease and disability alleviation for years to come. Prescribed hygiene and wound care methods, elevation, and exercises are currently the key self-care strategies for managing lymphedema of the leg in women $(5,32-34)$.

Neglected tropical diseases such as lymphatic filariasis requiring programs to both interrupt transmission of disease and implement morbidity control programs to prevent disfigurement, reduced productivity, and other sequelae of long-term illness strain already limited financial resources and public health infrastructure in resource-poor countries (1-3,9,35-37). Resource-poor countries are often faced with the goal of implementing lymphedema management programs to maximize health benefits from a fixed health budget already burdened with HIV/AIDS, malaria, tuberculosis, and other diseases associated with poverty, poor sanitation, and lack of clean water, lack of food, poor education, and poor housing. Despite the disparity between resources and needs healthcare and public health professionals and policy 
makers are increasingly being asked to partner with others in the development, implementation, and evaluation of evidenced-based, cost-effective public health programs that work. Best practices for public health interventions stress the need for systematic planning and evaluation with input from the intended recipients in order to tailor the intervention (38-41).

In this report, we use Intervention Mapping (IM), a framework for systematic health promotion program planning and evaluation, to develop program recommendations for self-care for women with lymphedema (41). IM has been used to guide development and evaluation of disease management programs that combine physical and psychosocial aspects of chronic disease including programs in management of cystic fibrosis, asthma management, urinary incontinence, and leg ulcers $(41-45)$.

We explain each step of the planning process (Figure 7.1) and then provide a case study example. The case study that is presented in each step of IM, includes information from a literature review of lymphatic filariasis and from a qualitative study of women with lymphedema of the leg living in filariasis-endemic areas of the southern coastal region of the Dominican Republic (DR) and in the rural fishing villages and farming communities in the southern coastal area of the Western and Central regions of Ghana (46-50). The case study is meant to illustrate how the IM process might be conducted in a country where women are at-risk of filariasis and what products would be produced in each planning step. By providing these concrete case examples, we hope to facilitate the adoption of systematic planning for this problem in countries where filariasis is endemic. 
Figure 7.1. Intervention Mapping steps and tasks (Adapted from Bartholomew et al. 2006)

\begin{tabular}{|c|c|}
\hline $\begin{array}{l}\text { Step } 1 \\
\text { Formative research and needs } \\
\text { assessment }\end{array}$ & $\begin{array}{l}\text {-Plan \& conduct a needs assessment for women with } \\
\text { lymphedema } \\
\text {-Assess quality of life for women with lymphedema } \\
\text {-Assess capacity of women and environmental agents } \\
\text {-Establish desired program outcomes }\end{array}$ \\
\hline $\begin{array}{l}\text { Step } 2 \\
\text { Create Change Matrices }\end{array}$ & $\begin{array}{l}\text {-State expected changes in behavior and the environment } \\
\text {-Specify lymphedema program objectives } \\
\text {-Specify determinants of behavior } \\
\text {-Create matrices of change objectives }\end{array}$ \\
\hline $\begin{array}{l}\text { Step } 3 \\
\text { Identify Theory-based Methods } \\
\text { \& Practical Strategies }\end{array}$ & $\begin{array}{l}\text {-Review program Ideas with women who have lymphedema } \\
\text { - -dentify theoretical methods that relate to women and their } \\
\text { behaviors } \\
\text { - Select or design practical strategies } \\
\text {-Ensure that practical strategies match change objectives }\end{array}$ \\
\hline $\begin{array}{l}\text { Step } 4 \\
\text { Develop Intervention Program }\end{array}$ & $\begin{array}{l}\text {-Consult with interested participants \& implementors } \\
\text {-Create scope, sequence, theme, \& materials list for the } \\
\text { comprehensive lymphedema program for women and change } \\
\text { agents } \\
\text {-Develop program documents \& protocols } \\
\text {-Pretest lymphedema management program materials with target } \\
\text { groups \& implementors, \& oversee materials production }\end{array}$ \\
\hline $\begin{array}{l}\text { Step } 5 \\
\text { Create Program Adoption \& } \\
\text { Implementation Plan }\end{array}$ & $\begin{array}{l}\text { - Identify program adopters and users } \\
\text {-Specify adoption, implementation and sustainability } \\
\text {-Specify determinants \& create matrix } \\
\text {-Select methods \& strategies } \\
\text {-Design interventions to affect program use }\end{array}$ \\
\hline $\begin{array}{l}\text { Step } 6 \\
\text { Create an Evaluation Plan }\end{array}$ & $\begin{array}{l}\text {-Describe the program } \\
\text {-Describe program outcomes } \\
\text {-Write questions based on matrices } \\
\text { - Write process evaluation questions } \\
\text {-Develop outcome indicators \& measures } \\
\text { - Specify evaluation design }\end{array}$ \\
\hline
\end{tabular}

\section{Recommendations}

\subsection{IM Step 1: Formative research and needs assessment}

\subsubsection{Step 1: Process and Products}

In Step 1 of the IM process, the program planner determines if there is a need for a public health intervention. Program planners conduct an assessment to better understand the science, the social implications, and the behavioral issues related to the health problem of interest. They also identify modifiable behavioral factors and environmental conditions and change agents that should be addressed in an intervention. In Step $\mathbf{x}$ the program planner brings together a planning group which should include members of the intended program participants and stakeholders from the communities at-risk and the healthcare and public health sectors. The planning group conducts a needs assessment and a health problem analysis among the population at-risk; assesses the capacities of the population and environmental 
agents to bring about change; and identifies program outcomes to link the assessment with the program evaluation. To begin, a literature search is conducted providing insight into the science to date and any existing programs that have been described in the literature. Both qualitative and quantitative methods of investigation can be used to better understand the experiences of the participants living with the health condition of interest.

The products of Step 1 include a logic model of the problem and program objectives.

\subsubsection{Step 1: Case Study}

\section{Methods}

In both countries the researchers brought together a planning group to conduct the needs assessment and health problem analysis. In Ghana the first author worked with healthcare and public health professionals and social scientists at the Health Research Unit, in the Ghana Ministry of Health. In the DR she also worked with healthcare and public health professionals from the Centro Para el Control de Enfermedades Tropicales (CENCET) and the Instituto Dermatológico de Cirugia y Piel (IDCP). The primary author and planning group first conducted an in-depth literature review of journal articles (1900-2006) and unpublished reports.

The review of the science informed a qualitative research study which included in-depth key informant interviews with 28 women in the Dominican Republic and 24 women in Ghana who had visible lymphedema to clarify the problems they faced, coping strategies they used, and identify additional modifiable behavioral factors for potential successful interventions. In addition, we recruited 24 Ghanaian women from Ministry of Health clinics for 3 focus group discussions (FGDs) and 28 Dominican women from the Instituto Dermatológico de Cirugia y Piel (IDCP) for 3 FGDs. We pre-tested qualitative interview FGD questions and terms with women with lymphedema in to assure correspondence with local dialects, cultural meanings, and women's understanding. They provided us with additional insights into the social implications, and the behavioral issues related to women living with lymphedema. They also provided insight into modifiable behavioral factors and environmental conditions and change agents that should be addressed in an intervention. Women were recruited for FGDs from clinic scheduling rosters and records, which included some women who were current clinic patients practicing medically prescribed self-care of their leg and some who were new patients with no previous prescribed self-care regimes.

Procedures included institutional and ethical review board approval from the Centers for Disease Control and Prevention (CDC), the Centro Para el Control de Enfermedades Tropicales (CENCET), and the Ministry of Health for Ghana. Interviewers read informed consent out loud and then the respondent signed with a signature or thumbprint. Interviews and FGDs were audiotaped, transcribed verbatim, translated into English by bilingual research assistants, and then entered as a Microsoft Word document into ATLAS-ti to facilitate text searching, data coding, and analysis (51).

We used open, axial, and selective coding to analyze the narratives. Findings derived from the needs assessment study that informed the IM process are described 
below. Member checking occurred with some women, whereby preliminary findings were discussed to validate researcher interpretations of women's accounts of living with lymphedema. Details of the qualitative study design, methods, and procedures are reported elsewhere (26-29).

In addition to the formal qualitative research, the first author talked to healthcare and public health professionals at the district and local levels, family members of women with lymphedema, field staff, indigenous healers, teachers, and medicinal shop owners and pharmacists.

\section{Needs Assessment Findings}

A summary of findings from the needs assessment are presented in an adapted PRECEDE model shown in Figure 7.2 (52). The figure depicts the nature of lymphatic filariasis and resulting impact on women's quality of life, positive and negative behaviors of the women related to lymphedema and its management, and environmental factors associated with either the behaviors of the women or with their health and quality of life directly. In the model the $(+)$ is associated with health promoting behaviors and the $(-)$ is associated with factors that were related to decreased health and function.

Beginning on the right side of the model in Figure 7.2 are aspects of chronic lymphedema, and the resulting impact on women's health and quality of life as described by women. In earlier stages of the disease process, women described lymphedema as an episodic health condition and failed to link episodes with a lymphatic filariasis cause (27). At later stages women reported frequent acute bouts of bacterial dermatolymphangioadenitis with the leg at increasing risk for open wounds, fungus, protuberances, massive size, gross disfigurement, and permanent disability (27). Women also reported chronic hip and back pain and sometimes severe lymphedema and complex conditions that required antibiotic prophylaxis to prevent sepsis. Some women used antibiotics without medical supervision, running the risk of developing antibiotic resistance (27).

Women also reported negative impact of lymphedema on their quality of life including psychological distress, stigma and fear of discrimination, related loss of self-esteem, negative body image, and social exclusion contributing to loss of social identify, and social connectedness (26-29). While women described loss of their livelihood and decreased economic productivity, Ghanaian women described greater hardship than Dominican women (28).

Moving to the left side of the model in Figure 7.2, women engaged in exhaustive and resource depleting attempts to find treatments and ultimately a cure for their lymphedema. They sought care and treatments from family, friends, indigenous healers and trained healthcare providers and reported more than 75 different home remedies and traditional practices. Many of these practices put the women at-risk of exacerbations of the condition. Women typically sought care from indigenous healers first and then care from medical sources when traditional practices were unsuccessful. The medical community also provided limited expertise in diagnosing, preventing, and caring for the condition. 
Figure 7.2. Needs assessment

Health Behaviors

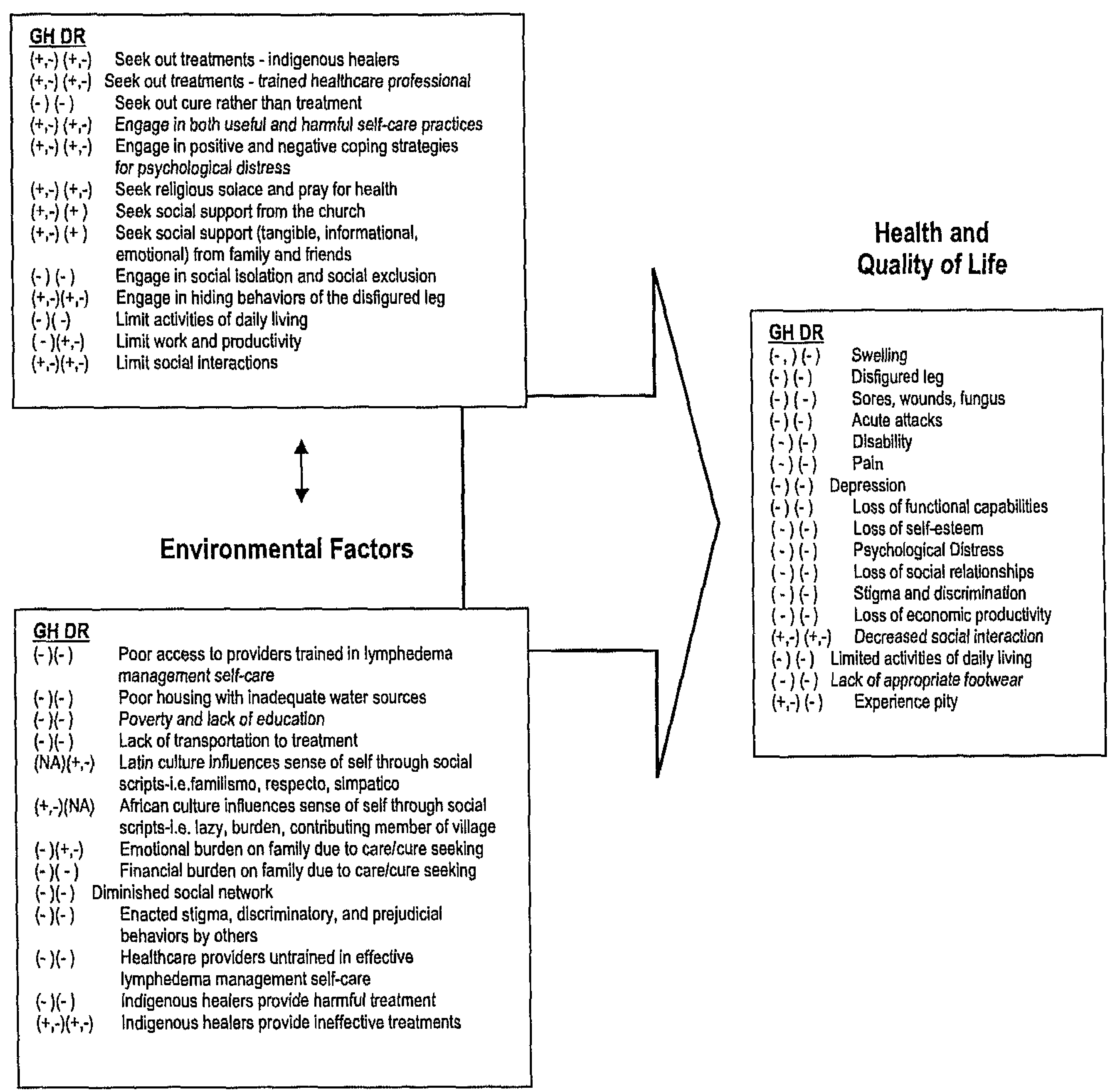

+ Indicates health-promoting behaviors

- Indicates behaviors associated with decreased health and function 
To deal with pervasive psychological distress, women used a variety of problem-focused and emotion-focused coping strategies. They talked about the importance of religion, faith, and prayer for coping with their condition. Many women hid their leg from others, were unable to wear socially acceptable clothing or shoes and were socially isolated or actively excluded.

Dominican women often sought social support (tangible, informational, emotional and companionship) from family, friends, and the church (29). Ghanaian women rarely sought out social support but were sometimes provided for by family and occasionally by church members. They were less likely to speak about the need to belong or seek out others in order to overcome feelings of loneliness and alienation. Ghanaian women described a greater sense of resignation over their situation than Dominican women (29).

Women described a spectrum of stigma experiences including enacted, perceived, and felt stigma that contributed to discriminatory social interactions, social exclusion behaviors, low self-esteem, and psychological distress. Behaviors to cope with stigma could be either protective or harmful. For example, women anticipating potential stigmatizing behaviors by others engaged in self-isolation which often contributed to feelings of loneliness and depression. A few Ghanaian women, when at their most depressed, had even thought of ways to kill themselves such as hanging themselves or eating rat poison. Fewer Dominican women reported suicidal thoughts (28).

Limited access to health providers trained in lymphedema management along with stigmatizing behavior by the providers; lack of access to clean water, lack of transportation, and lack of money for supplies and medications were some of the environmental barriers to self-care as described by women. In addition single or widowed women with severely disfigured and grossly enlarged legs often lacked the capacity to move through the community to seek care and resources such as clean water and medications and had little social support to assist them.

The experiences of women with lymphedema exist within a cultural context of cultural scripts, social norms and expectations influence these experiences. For example, in Ghana the cultural script suggesting that people who don't work are lazy often defined how women with severe functional limitations were viewed by the community. In the DR complaining about a problem or being a burden to one's family were unacceptable and reduced health seeking and social connectedness among women (28-29).

Women in the Dominican Republic had greater access to education, healthcare, jobs, and family resources than women in Ghana. Dominican women also had a higher standard of living, higher levels of education, and greater availability of social services. Ghanaian women had poorer housing and less access to clean water and more often had to travel greater distances to reach health facilities. Ghanaian women were more likely to report that people pitied them but associated pity with people providing money and other items to assist in meeting their needs. Dominican women described feeling pity from others as a degrading experience associated with feelings of stigma.

Despite disparity in resources, women in Ghana and the Dominican Republic shared many similar experiences in living with lymphedema and elephantiasis of the 
leg. The combined results of this process in Step 1 strongly supported the need for a public health intervention for women with lymphatic filariasis to address self care and enhance coping skill (26-29). In addition the needs assessment noted that healthcare providers were poorly trained to diagnose and treat women; indigenous healers were generally misinformed about the condition and poorly linked to the healthcare system; community awareness about early diagnosis and care of lymphedema was minimal or absent; support and education for members of women's natural helper networks were minimal or absent; and tailored educational campaigns and activities to reduce stigma were nonexistent.

\subsubsection{Step 1: Case Study Limitations}

In this case example, the working group for the formative research did not continue beyond the needs assessment. Steps 2-6 of IM were conducted post-hoc by the team of authors. Whereas, when IM is conducted in a real planning situation, a more diverse work group would have been established and continued throughout the planning process. Another limitation of this assessment was that husbands and other members of the women's environment such as indigenous healers, church members and informal network members were not formally interviewed. Including these people could have provided greater insight into the needs of women and the possible contributions of the environment. Mapping community assets and assessing community capacity would have strengthened the needs assessment and provided insight into the social and physical infrastructure that could provide support to women and their natural helpers and contribute to more sustainable interventions.

\subsection{IM Step 2: Matrices of change objectives}

\subsubsection{Step 2: Processes and Products}

In Step 2, the program planner systematically creates matrices of change objectives which will serve as the foundation of the intervention by specifying whom and what should change among the intended population and interpersonal and community change agents as a result of the recommended intervention. The planning group begins by reviewing the findings from the needs assessment to identify behavioral and environmental factors that can be changed through a public health intervention and stating the program outcomes for each. Next, the planners develop performance objectives by asking "What would participants in the program need to do to create the behavioral and environmental change?" The performance objectives are then associated with theory-informed, modifiable determinants of behavior and environment. Determinants are derived from both the needs assessment and problem analysis and from the literature regarding what factors are known to be related to the types of behavior change specified in the performance objectives.

Products of this step include specific statements of what should change in the intended population and their environment; lists of detailed performance objectives and matrices of change objectives that describe change required in determinants in order for the specified performance to be achieved. 


\subsubsection{Step 2: Case Study}

\section{Proposing What Should Change}

Findings from the needs assessment supported the proposed changes in behavior of women, healthcare providers, and others who interact with women. Change for the women focuses on using effective self-care practices and coping strategies. In Table 7.1, recommended change for trained healthcare providers focuses on adequate diagnosis and treatment, indigenous healers on doing no harm and referral of lymphedema complications, and women's networks and community on reduction of stigma and provision of social support. The change recommendations for environmental actors were derived from women's reports during the needs assessment, along with information obtained through informal discussions with family members and friends of women, clinic and field staff, indigenous healers, teachers, and medicinal shop owners and pharmacists. The left column of Table 7.2 presents the detailed activities (performance objectives) that would be required of women to achieve the behaviors listed in Table 7.1.

Table 7.1. Behaviors of women with lymphedema and persons in the environment

\begin{tabular}{|c|c|c|c|}
\hline & What needs to $\mathrm{c}$ & lange in the behavior o & change agents? \\
\hline $\begin{array}{l}\text { Women } \\
1 . \text { Engage in } \\
\text { efficacious and non- } \\
\text { risky self-care } \\
\text { practices for } \\
\text { lymphedema of the } \\
\text { leg along with } \\
\text { efficacious coping } \\
\text { practices for } \\
\text { management of } \\
\text { psychosocial } \\
\text { distress. }\end{array}$ & $\begin{array}{l}\text { Healthcare Providers } \\
\text { 1. Diagnose and } \\
\text { manage lymphatic } \\
\text { filariasis and resulting } \\
\text { physical and mental } \\
\text { consequences } \\
\text { 2. Instruct women in } \\
\text { efficacious self care } \\
\text { and coping practices to } \\
\text { replace risky or harmful } \\
\text { self-care and } \\
\text { inefficacious coping. } \\
\text { 3. Promote education } \\
\text { and stigma reduction } \\
\text { strategies to decrease } \\
\text { disease-related stigma } \\
\text { from family, friends, } \\
\text { community members, } \\
\text { and healthcare } \\
\text { providers. }\end{array}$ & $\begin{array}{l}\text { Indigenous Healers } \\
\text { 1. Incorporate teaching } \\
\text { efficacious self-care } \\
\text { practices into their } \\
\text { healing activities to } \\
\text { support women who } \\
\text { seek care through } \\
\text { traditional care } \\
\text { networks. } \\
\text { 2. Refer complications } \\
\text { that may require } \\
\text { medical interventions to } \\
\text { trained healthcare } \\
\text { providers. } \\
\text { Educators } \\
\text { 1. Promote education } \\
\text { and stigma reduction } \\
\text { strategies in the school } \\
\text { and community to } \\
\text { decrease disease- } \\
\text { related stigma from } \\
\text { family, friends, and } \\
\text { community members }\end{array}$ & $\begin{array}{l}\text { Members of Women's } \\
\text { Natural Helper Network } \\
\text { 1. Provide social } \\
\text { support (e.g. emotional, } \\
\text { instrumental, } \\
\text { informational and } \\
\text { appraisal) to women } \\
\text { with lymphedema } \\
\text { 2. Provide advice and } \\
\text { support for efficacious } \\
\text { self-care practices and } \\
\text { coping strategies into } \\
\text { natural helper networks }\end{array}$ \\
\hline
\end{tabular}




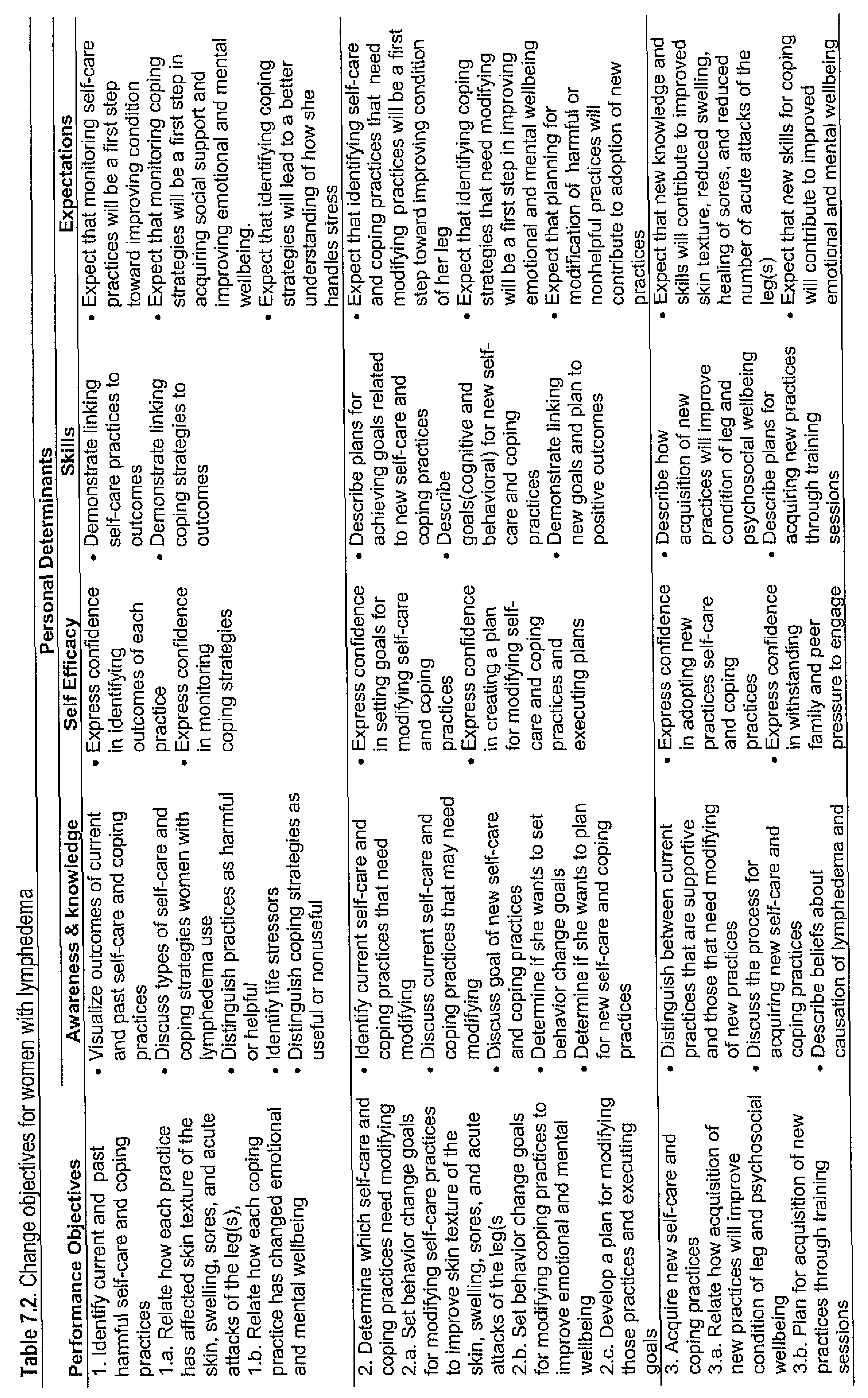




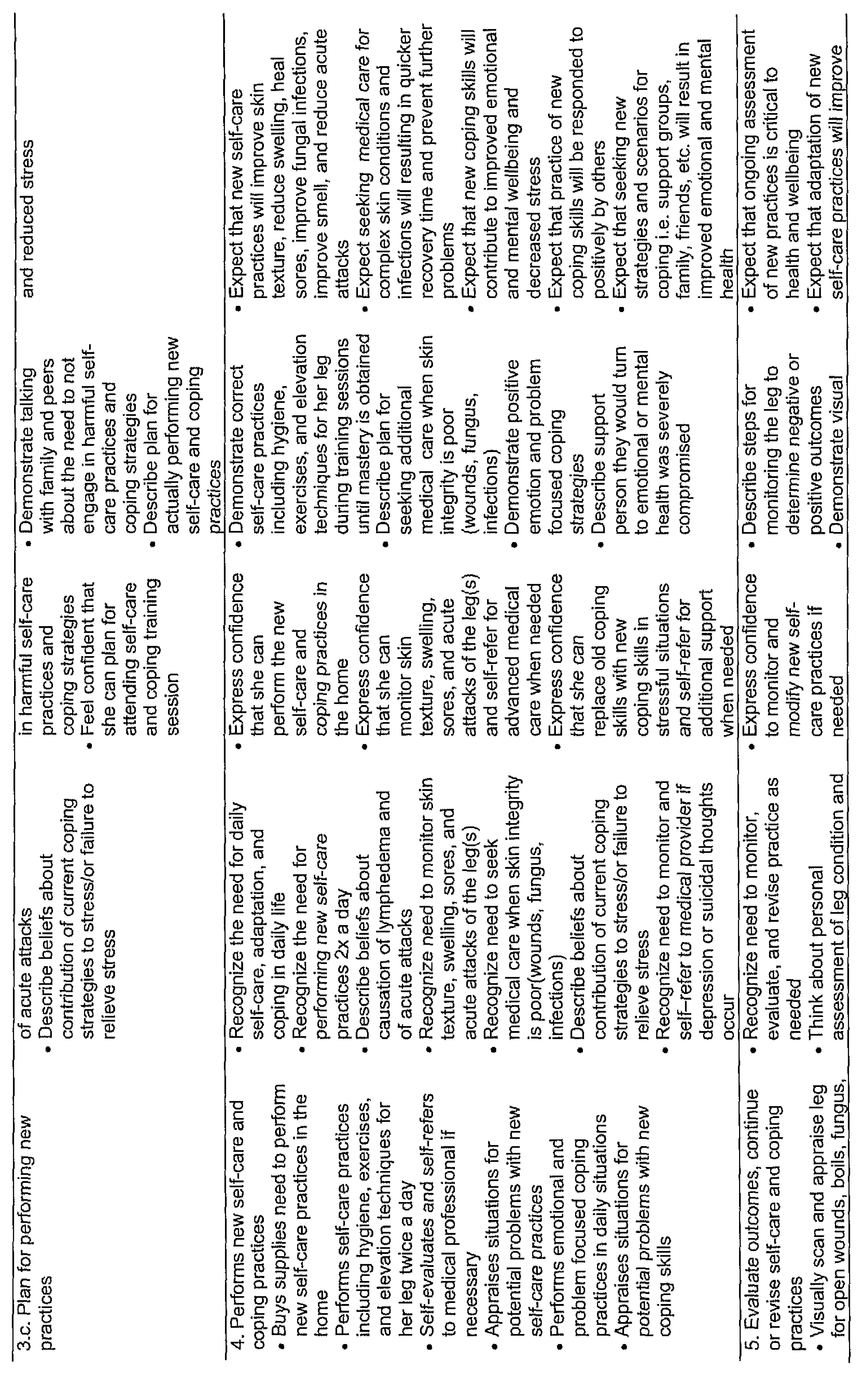




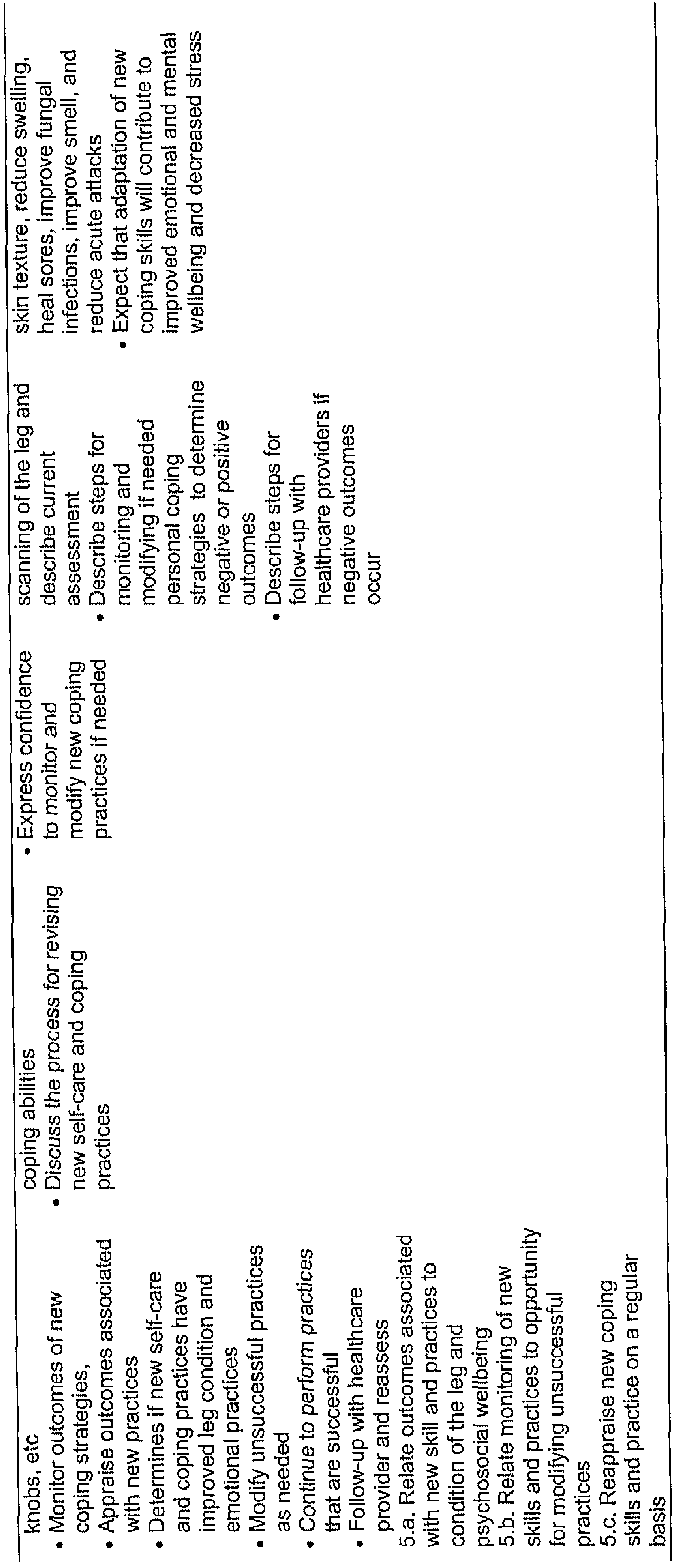


Performance objectives for women were based on the discussions with the women and on self-regulation theory, a behavioral model that has been used to guide development of intervention programs in asthma management (52), rheumatoid arthritis (53), and diabetes mellitus (54). In the theory people are described as directing and controlling their actions in order to acquire information and expertise (55). Behavioral self-regulation theories are typically described by a set of processes such as 1) setting goals; 2) self-observation to assess and monitor current behaviors; 3) planning, setting, and developing strategies to reach goals; 4) engaging in new behaviors and monitoring progress, and 4) evaluating outcomes, and modifying strategies (55-59).

The performance objectives are then associated with theory-based, modifiable behavioral determinants to create change objectives. Change objectives specify who and what will change as a result of the proposed intervention. Change objectives, more specific than traditional program goals and objectives, are presented in a simple matrix of change to depict the person who should change along with the performance objectives paired with suggested behavioral determinants of the performance that must be influenced for change.

The left column of Table 7.3 presents the same level of detail for performance objectives for one example from the women's environment-the healthcare provider.

\section{Determinants of the Proposed Performance Objectives}

Across the top of Tables $\mathbf{7 . 2}$ and $\mathbf{7 . 3}$ are theoretical determinants of the performance objectives in the left column. These determinants address "why" questions: Why might the women perform the behaviors? Why might the healthcare providers perform the behaviors? Drawing upon the findings and field notes from the needs assessment the determinants of awareness and knowledge, self-efficacy, skills, and outcome expectations consistent with the constructs from Social Cognitive Theory were identified as influential and changeable behavioral determinants for women (57-58). Social Cognitive Theory, a behavioral theory based upon active learning promoting performance through the learning process has been used in public health interventions with such conditions as HIV/AIDS prevention (59) asthma, (60) and heart disease (61). While social support from members of women's natural helper network (family and friends) was identified as a determinant for coping it was also identified as a behavior that needed attention in the environment of women. Many women lacked knowledge about their condition and relied on traditional beliefs for the cause of the condition and subsequently used indigenous healers as their first sources of care and treatment. Most women also lacked or had limited knowledge and awareness of potentially health promoting, self-care behaviors for lymphedema of the leg (27). Additionally, most women expressed little self efficacy (confidence) and little outcome expectation that changing their current behaviors and improving self-care skills would produce a change in their lymphedema, particularly the integrity of their skin. Management of their leg in its current condition and prevention of further complications were not seen as important health goals among many women in either country. Women were typically seeking a cure for their leg(s) to return to their previous size and texture. Some women also lacked access to healthcare and clean water for self-care. 


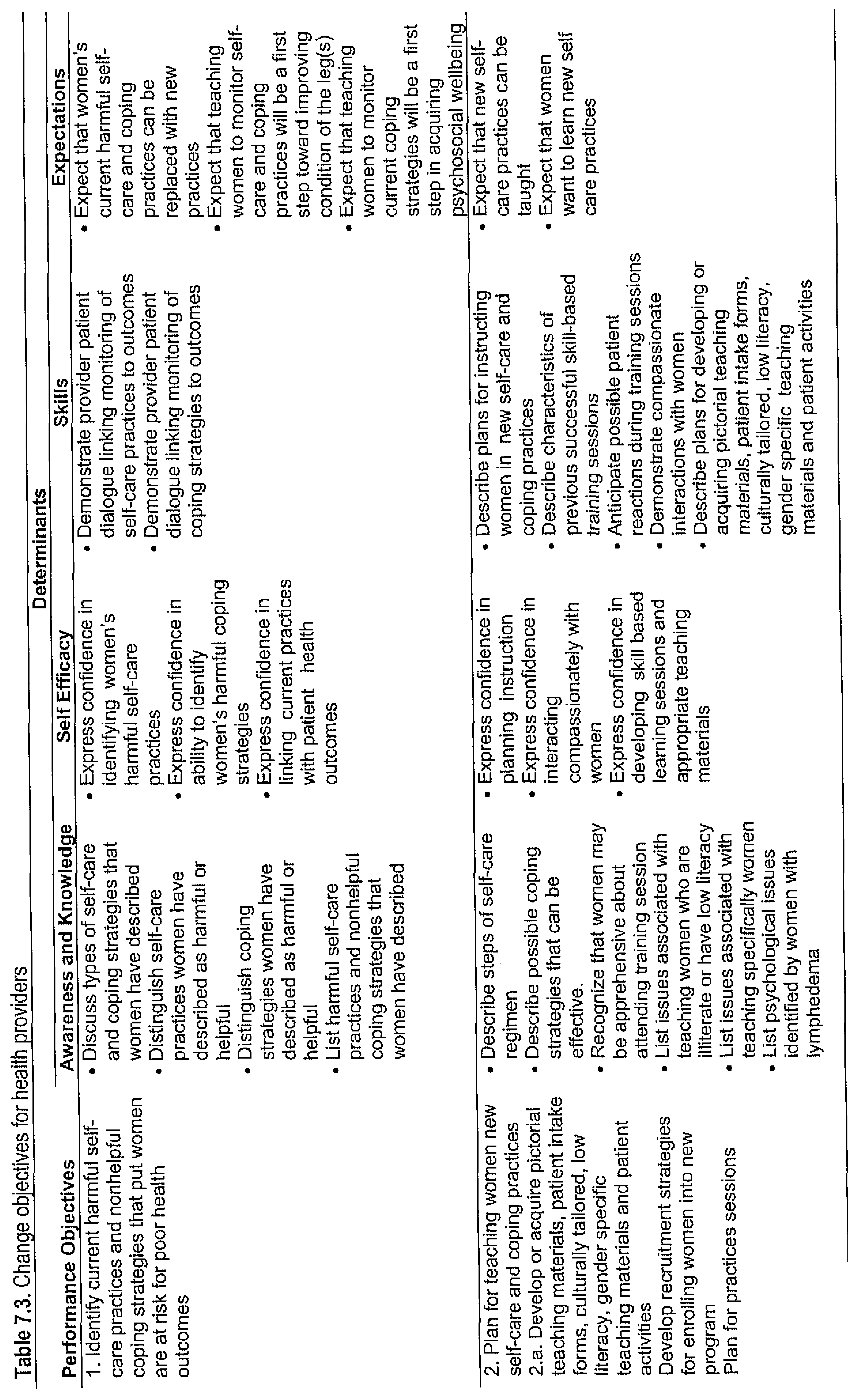




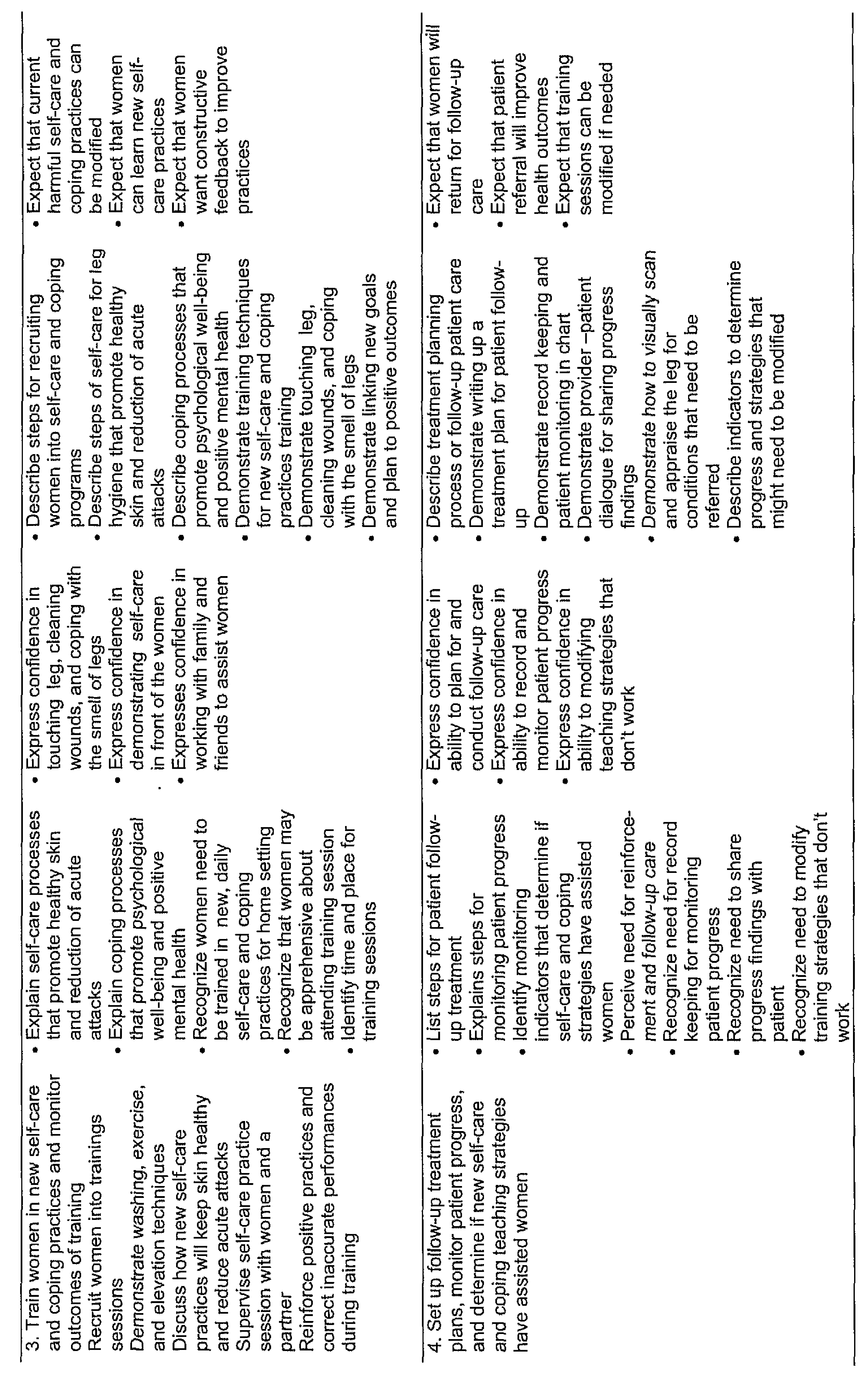




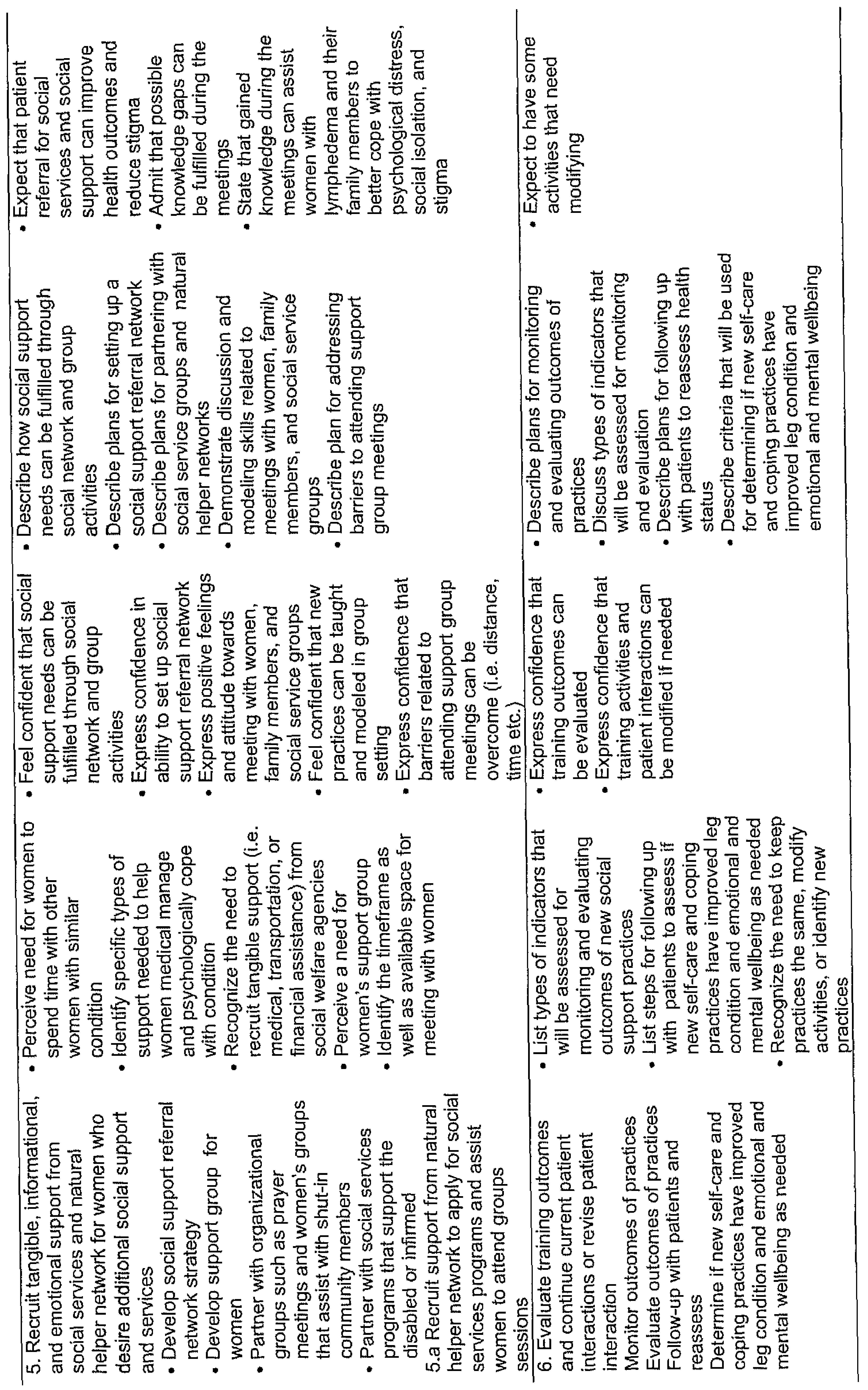




\section{Matrices}

Matrices are the foundation for program development. They are formed by crossing the performance objectives with determinants of behavior. The guiding question to complete the cells on Table 7.2 was: What would the woman have to change (about the column head determinant) in order to achieve the performance objective in the left column. For example, looking at the column with the determinant of self-efficacy, we asked: What would women have to change regarding their self-efficacy in order to identify current and past harmful self-care practices (first performance objective from left column). An answer would be: women with lymphedema (population) express confidence (determinant: self-efficacy) that they can identify the outcomes of each practice used to care for their leg (performance objective). These answers that combine information on the performance and the determinant complete the cells of the matrix. An additional example would be healthcare providers providing services to women with lymphedema (population) would expect that current harmful self-care and coping practices can be modified (determinant: outcome expectations) and train women in new self-care and coping practices and monitor outcomes of training (performance objective). Additional matrices of change for indigenous healers, educators, and members of natural networks are available from the primary author.

\subsubsection{Step 2: Case Study Limitations}

More in-depth systematic exploration into healthcare and public health professionals, indigenous healers, educators, and members of natural helper networks knowledge, attitudes, and skills would have strengthened this step.

Another weakness is the use of theories most often derived from situations in developed countries and not validated in resource-poor countries. Although Bartholomew et al. along with Resnicow and colleagues posit that ... "theories are descriptions of processes that can be generalized over groups and over cultures" (41). And while many theories have yet to be rigorously validated in resource-poor countries, they have increasingly been used in such settings with growing success when the cultural context (superficial and deep) of the affected population and the behavior of interest has been thoroughly considered.

Another limitation of Step $\mathbf{2}$ is the relatively narrow focus on factors to change. An important factor not addressed here is the lack of a referral system to address medical management of complicated cases and mental health support. Other considerations might be poverty, lack of access to clean water, gender inequities related to education and violence, and other critical factors that may be related to the women's ability to manage lymphedema and the capacity of communities to help them.

\subsection{IM Steps 3 and 4: Methods, Strategies and Program Components}

\subsubsection{Steps 3 and 4: Processes and Products}

In Step 3, the program planning group identifies theory-based intervention methods and practical strategies designed to influence the change objectives from the matrices in the previous step. In this step, it is important to review program ideas with the intended participants and use their perspectives when identifying methods 
and strategies. Working from the matrices, the planning group identifies theoretical methods that can influence change in determinants and identifies the conditions under which a given method is most likely to be effective. Then, the theoretical change methods must be translated into practical strategies that can be organized in Step 4 into a program and delivered to the various program participants. Finally, the planners should ensure that the final strategies contain methods that will influence the change objectives in the matrices (41).

Bartholomew and colleagues (2006) describe methods and strategies as a continuum, abstract theoretical methods, through practical strategies, to organize programs. For example, "skills training with guided practice and feedback" is a theoretical method; a step-by-step instruction from a healthcare provider is a practical strategy to deliver the skills training, and a program would describe when and how the training would be delivered.

The product of Step 3 is a description of methods and strategies that will be used in the program linked to the change objectives they will influence. In the next step, the methods and strategies will be the basis of intervention components that can be delivered to the priority population and the environmental agents. In Step 4 the methods and strategies are combined into program components and organized into a deliverable program scope and sequence. Also in this step the planners produce the necessary protocols, materials, teaching tools and products for the program to be implemented. Program materials are pretested with the intended population and modifications are made if necessary. Program implementers are consulted to determine feasibility of the proposed intervention components.

\subsubsection{Steps 3 and 4: Case Study}

\section{Methods, Strategies and Materials}

Working from the matrices, we proposed theoretical methods and practical strategies in Step 3. In Step 2 behavioral determinants of knowledge and awareness, self-efficacy, skills building and outcome expectations were identified as being influential and changeable behavioral determinants for women. In Step 3 we linked these with such theoretical methods such as personal risk appraisal, motivational interviewing, modeling, goal setting, and active learning. The theoretical methods were further linked with practical intervention strategies such as orally presenting woman with scenario-based vignettes of risky behaviors that lead to poor skin condition, infection, and acute attacks and discussing with them their own risk of such problems. Engaging women in setting realistic goals and expectations that they believe are possible, and having women provide testimonials on the benefits of new self-care behaviors are further examples of practical intervention strategies which would link to the theoretical methods presented above.

During the needs assessment, the primary author and interviewers participated in experiences that led to ideas about methods, strategies, and program components. When interviewing women it was noted that many women were illiterate and not comfortable interpreting cartoon or simple line drawings. Following each key informant interview and FGD women were provided local soap and taught lymphedema self-care skills. Women practiced and discussed the steps of self-care and were provided feedback and positive reinforcement. The primary author also 
created several educational tools that women reviewed. Women identified a simple leg care manual with instructional photographs and very few words as a desirable educational tool for learning how to care for their leg. Utilizing local soaps, skin care ointments and medications contributes to feasibility of the program. The importance of simple instruction, demonstration, and enactment by the women, feedback and encouragement were incorporated into the suggested methods and strategies in Table 7.4.

In Table 7.4, we present the determinants to be influenced, the underlying theoretical method, and the practical strategy with the materials or tools needed for the strategy to be successfully administered to women in a lymphedema management program. For example, to address the change objectives related to the determinant of skills acquisition, the recommendation is skills training, guided practice with feedback for leg and wound care, and positive reinforcement. One practical strategy might be step-by step instruction by a woman with lymphedema during practice sessions where women actually wash, dry, and care for the wounds on their leg and get reinforced and complimented on their efforts.

Suggestions for program components which address the needs of healthcare and public health professionals, educators, indigenous healers and members of women's natural helper network were drawn from informal discussions with members of the intended audience, the literature related to behavioral interventions and clinical care settings in developing countries, and previous field and clinical experiences of the authors. Clinic and field staff also felt that a pocket size pictorial notebook with photographs of fungal infections, wounds and skin conditions with appropriate medical management steps and referral options would be a good tool for them as well. In Table $\mathbf{7 . 5}$ we engage in the same process for matching theoretical methods and practical program strategies to the proposed change objectives for healthcare and public health professionals who would care for women with lymphedema. Recommendations for indigenous healers, the women's natural helper networks, and educators are available from the first author.

\section{Recommended Program Components, Scope and Sequence}

In Step 4 the strategies identified in IM Step 3 are incorporated into the design of a comprehensive lymphedema management program. The program in Table 7.6 presents the scope and sequence of specific program activities, messages and delivery mechanisms. The recommended public health program is multicomponent. One component focuses on influencing environmental conditions and social interactions of women through the education and training of such people as healthcare providers in order to improve their ability to diagnose and manage the physical and psychological sequelae of disease, indigenous healers on doing no harm and referral of lymphedema complications, and women's networks and community on reduction of stigma and provision of social support. The program also includes a component specifically aimed towards women with sequelae of lymphatic filariasis to support them in using effective self-care practices and coping strategies. 


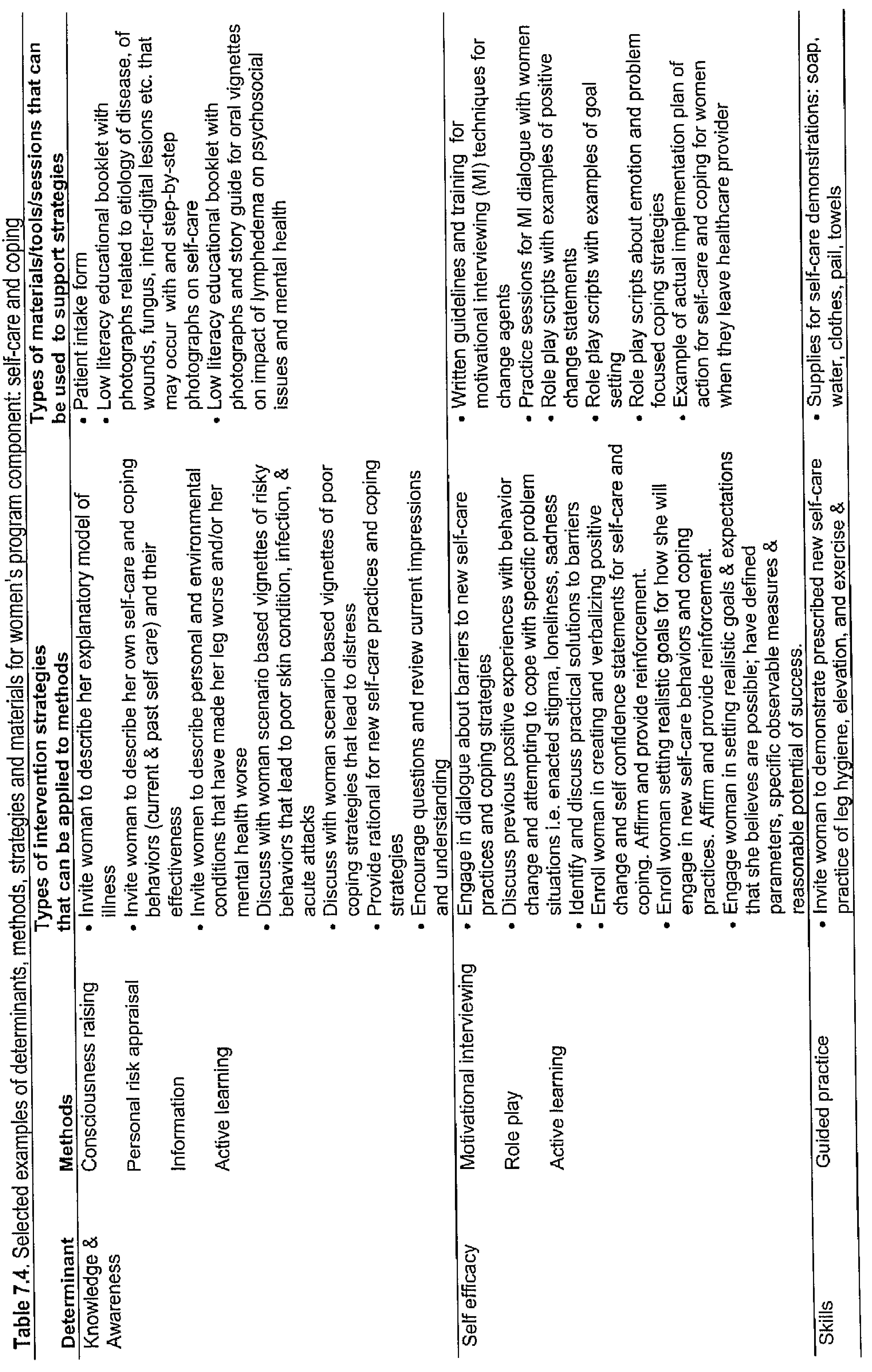




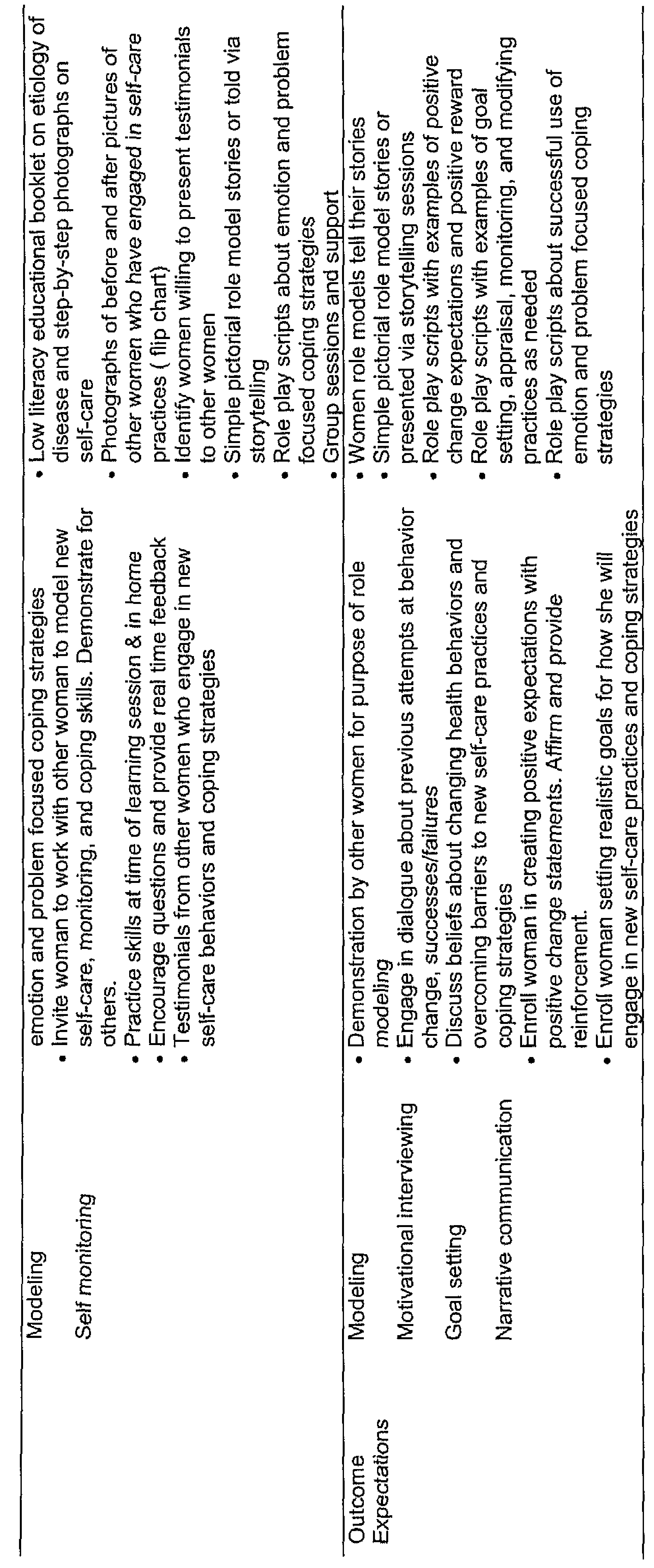

122 
Table 7.5. Selected examples of determinants, methods, and strategies for program component: training of healthcare and public health professionals

\begin{tabular}{|c|c|c|c|}
\hline Determinant & Methods & $\begin{array}{l}\text { Types of intervention strategies } \\
\text { that can be applied to methods }\end{array}$ & $\begin{array}{l}\text { Types of materials/tools/ } \\
\text { sessions that can be } \\
\text { used to support } \\
\text { strategies }\end{array}$ \\
\hline $\begin{array}{l}\text { Awareness } \\
\text { Knowledge } \\
\text { Self-efficacy } \\
\text { Skills } \\
\text { Expectations }\end{array}$ & $\begin{array}{l}\text { Consciousness } \\
\text { raising } \\
\text { Active learning } \\
\text { Role modeling } \\
\text { Guided } \\
\text { practice } \\
\text { Community } \\
\text { mobilization }\end{array}$ & 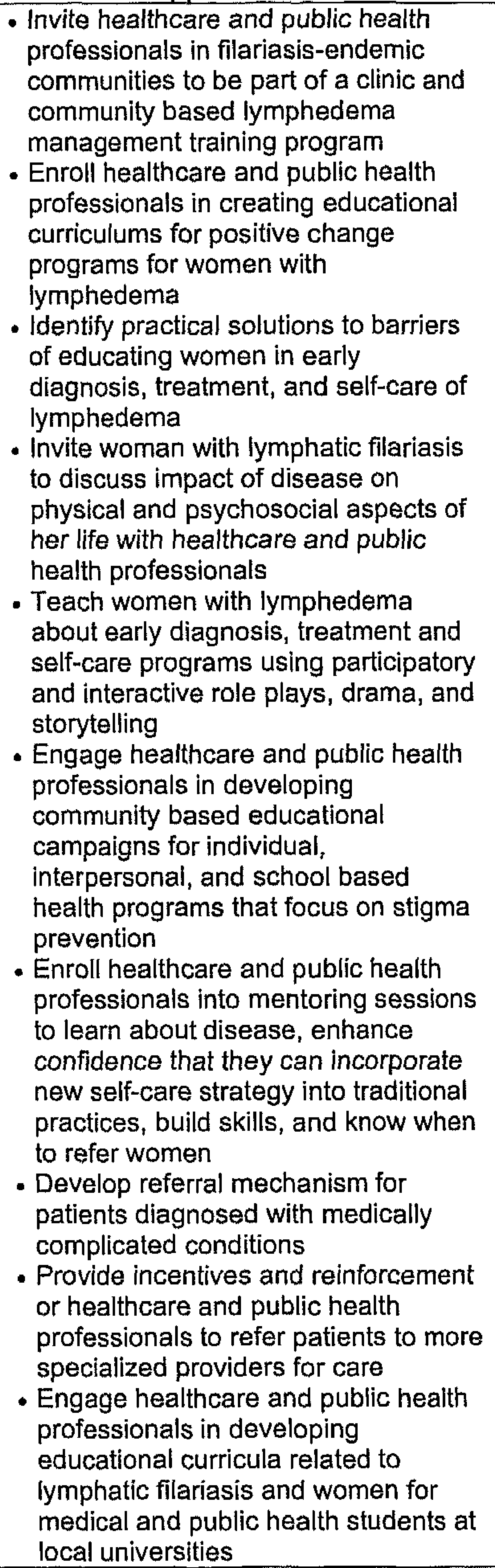 & $\begin{array}{l}\text { - Engage in dialogue with } \\
\text { invitation to participate } \\
\text { - Training curriculum and } \\
\text { training sessions for } \\
\text { healthcare and public } \\
\text { health professionals in } \\
\text { filariasis-endemic } \\
\text { communities } \\
\text { - Role play scripts for } \\
\text { healthcare and public } \\
\text { health professionals about } \\
\text { transmission of disease, } \\
\text { prevention activities, and } \\
\text { encouraging others to } \\
\text { seek early diagnosis and } \\
\text { treatment } \\
\text { - Role play scripts for } \\
\text { healthcare and public } \\
\text { health professionals about } \\
\text { emotional and mental } \\
\text { health issues associated } \\
\text { with lymphedema among } \\
\text { women } \\
\text { - Role play scripts about } \\
\text { stigma and stigma } \\
\text { prevention } \\
\text { - Educational booklet of } \\
\text { vignettes tailored to stage } \\
\text { of lymphedema for use } \\
\text { with woman } \\
\text { - Guidelines for university- } \\
\text { based activities to create } \\
\text { awareness } \\
\text { - Woman with lymphedema } \\
\text { willing to talk at training } \\
\text { sessions programs }\end{array}$ \\
\hline
\end{tabular}




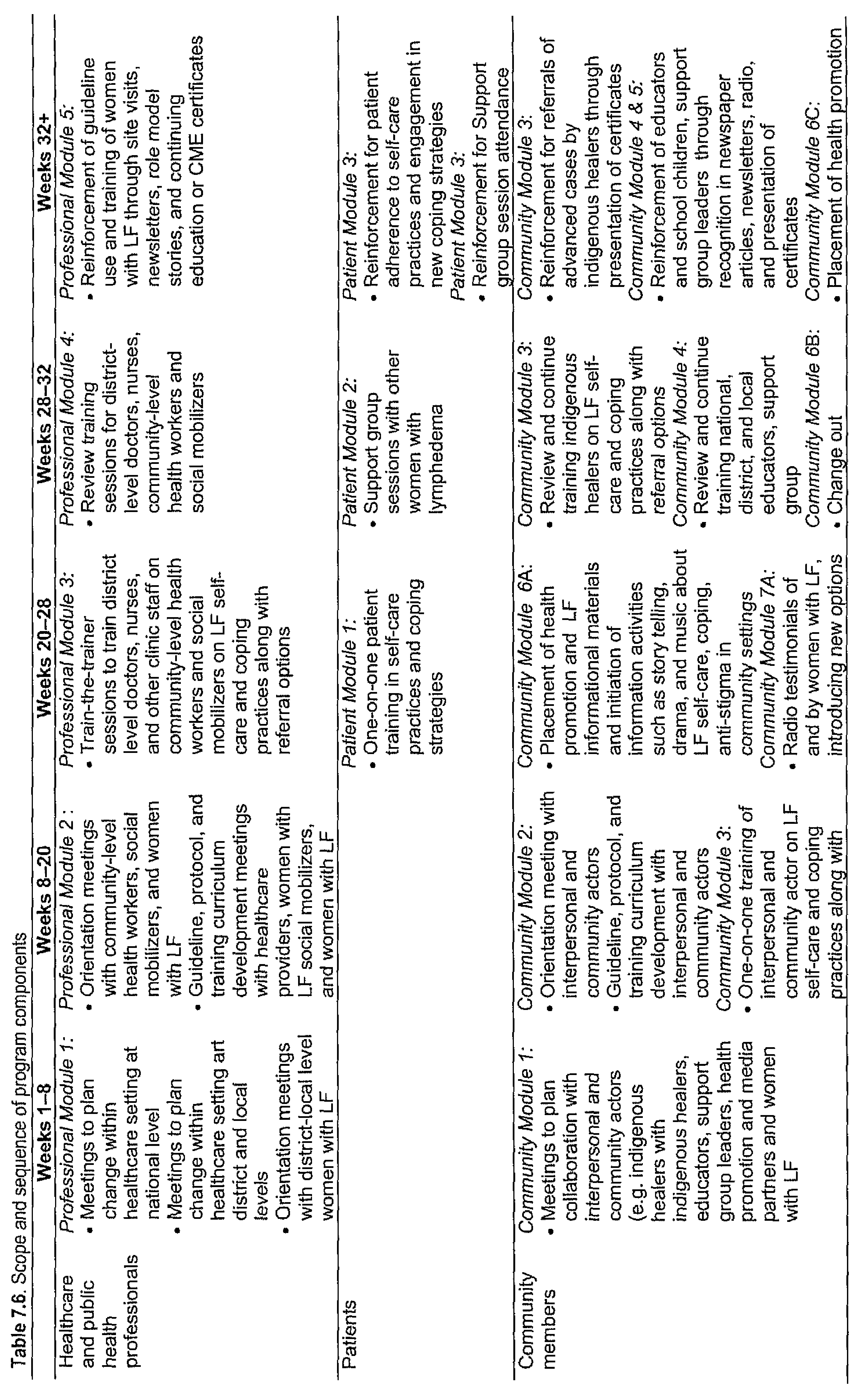




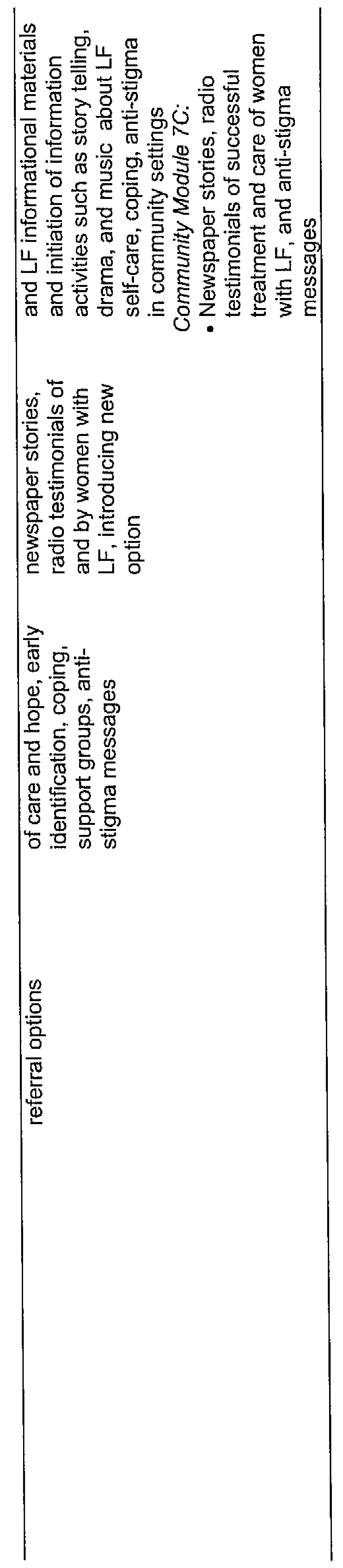


Healthcare professional modules include planning meetings; orientation sessions; development of guidelines, protocol, and curriculum; train-the-trainer sessions; review sessions for modification of activities; and finally reinforcement and positive support activities for program implementers. Modules would provide detailed objectives, tasks, and activities that address the needs of women as identified in the original needs assessment. For example, training clinic and field staff how to take a good history would include how to encourage women to share any traditional self-care practices that they might be practicing and to determine whether they were harmful or not. A good history would also include identifying any emotional or mental health issues that might be affecting women at the time of their visit. The modules for the women with lymphedema comprise in-clinic training and ongoing reinforcement and support group sessions. The community program modules include planning and orientation sessions leading to training activities for indigenous healers, educators, and support group leaders for women and member of their natural helper networks. The community modules also contain community health promotion activities and mass media efforts.

The sequence of activities presented covers an 8-month time period for professional development and training sessions to improve knowledge, self-efficacy, skills and outcome expectations of program implementors to implement the program as designed for women with lymphedema as well as setting up the infrastructures for program activities. The timeframe could be extended without significant detriment to the program for countries who often contend with resource-poor infrastructure, political instability, and logistical barriers (e.g heavy rains, poor roads, uncertain electricity). Innovative thinking is critical for adapting prescribed skin care regimes that require washing with clean water when water is scarce or not clean.

\subsubsection{Steps 3 and 4: Case Study Limitations}

The process of Steps 3 and 4 was not grounded in continued experience with the in-country working group of Step 1. In a real situation, an in-country planning group would comprise multiple stakeholders including the women with lymphedema. The program recommendations presented here must be considered hypothetical and if adopted, should be adapted by a planning group in the country preparing a program.

\subsection{IM Step 5: Planning for program adoption, implementation, and sustainability}

\subsubsection{Step 5: Processes and Products}

In Step 5, the program planner creates a plan specifying the processes to support program adoption, implementation and sustainability. A primary focus of this step is linking the designers of the new intervention program with those who will implement the program. If the planner has been true to the IM process they will already have a planning group of appropriate individuals to determine the best process for program implementation. Sustainability or institutionalization of the program is another important focus of Step 5 if the program is to have measurable impact over time. Therefore it is important that influential members from the group 
of program adopters (e.g. ministry of health officials, private and public healthcare providers, non-governmental partners and stakeholder) be part if the initial IM planning group. It is also important that program adoption, implementation, and sustainability processes be considered from the beginning of the IM process.

The products of this step are performance objectives for program adopters and implementers; a matrix of change objectives for adoption, implementation and sustainability; and a plan to influence the change objectives on the matrix.

\subsubsection{Step 5: Case Study}

Similar to Steps $\mathbf{3}$ and $\mathbf{4}$ the recommendations we present are not derived from the participatory process prescribed by the IM framework but presented as program recommendations for the reader's consideration. In this step we describe how one might translate the recommendations from our example into settings where they are needed. For an agency or organization adopting and implementing the program recommendations derived from our case study examples, the completion of program components will require working with the women and healthcare and public health professionals to identify the activities that are most appropriate to the local social and cultural context as well as develop and translate materials. A first step of adapting these recommendations to meet the specific needs of women is the development of linkages between promoters of the program, people who would be in a position to run the program day-to-day, and the women with lymphedema.

One possible model would be to establish a Ministry of Health ( $\mathrm{MOH})$ planning committee which optimally would include healthcare and public health professionals at the local, district and national levels as well as women with lymphedema, teachers, family members, and indigenous healers to ensure a good fit between the program, its implementers, and the affected women. Professional team members can determine if lymphedema management care is integrated into a general health clinic or stand alone clinic to address the special needs of women. MOH professionals partnered with women with lymphedema can train district and local health management teams as well as indigenous healers to adapt the self-care and coping program to fit the needs of each health center or program, creating ownership, and addressing the specific needs of women in their communities. Referral mechanisms would need to be developed for complicated medical problems and mental health conditions as well as support mechanisms for natural helper networks. Following the development of linkages the planning committee would identify performance objectives to delineate what decisions makers and program implementers need to do to ensure program implementation and to write an implementation plan. Similar to IM Step 2 the development of performance objectives leads to the specification of determinants of behavioral and environmental conditions that would promote program adoption such as awareness of the program among those people who have to manage and implement the program in-country. An example of performance objectives for adoption and implementation is presented in Table 7.7. 


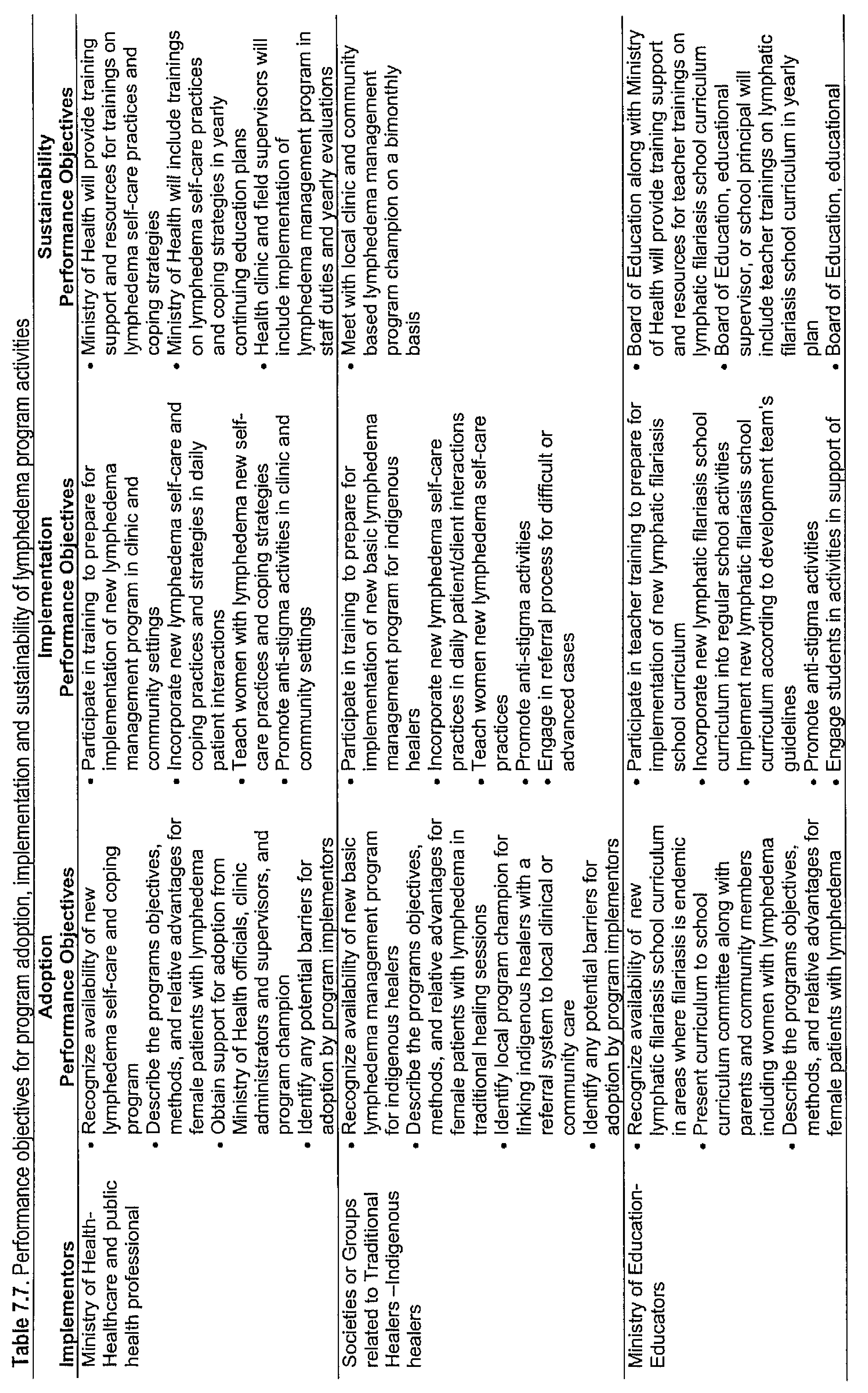




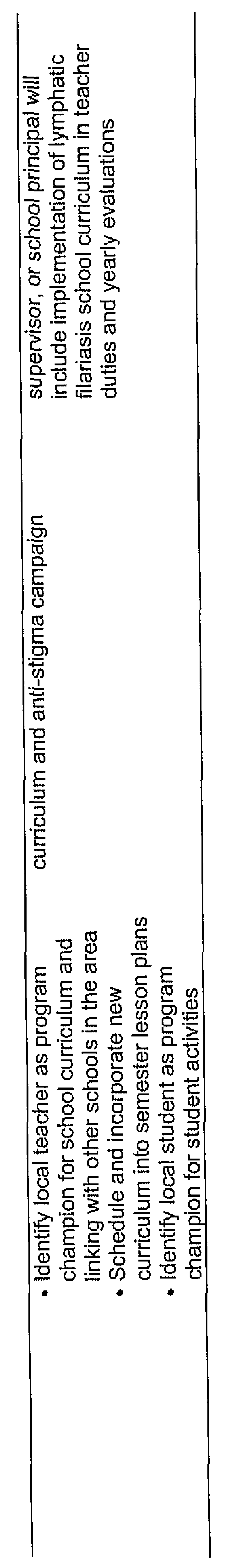


To proceed with matrix development in this step, planners would talk to the individuals and groups who are proposed to participate in implementation to begin to understand determinants related to participation. Similar to Step 2, this requires asking a why question. For example: Why would you establish a lymphedema program hosted by the MOH? Knowing these factors that may be idiosyncratic on a country level or on a more local level is important before constructing a matrix and planning methods and strategies to influence program adoption.

\subsubsection{Step 5: Case Study Limitations}

In this step, we provided recommendations for beginning a program in a country. We are unable to complete this case study step without information from a specific country that is considering program adoption. We did not complete this step with the two case study countries.

\subsection{IM Step 6: Recommendations for program evaluation}

\subsubsection{Step 6: Processes and Products}

In Step 6, the program planner describes the proposed program linked with program outcomes. The program planner also specifies the evaluation design and, drawing upon the matrices presented in the previous steps, develops outcome indicators and measures with accompanying process and outcome questions.

The product of this step is an evaluation plan that capitalizes on the output from all previous IM steps.

\subsubsection{Step 6: Case Study}

In this step we first developed a logic model for the program recommendations presented here (Figure 7.3). To use the logic model for evaluation, we examined each column to formulate evaluation questions. For example, under patient identification and recruitment within the column called project activities, program staff should seek out women with "big legs," screen them to distinguish lymphedema from non-lymphedema, and refer women for treatment and self care training. Process evaluation questions pertaining to this column might include: What were the components of the recruitment strategy for identifying women with a big leg? Who and how many people were involved in recruitment? What as the protocol for distinguishing filarial lymphedema from non-filarial lymphedema? Then as a result of the patient identification and recruitment program component, in the outputs column, questions might pertain to: How many women have been identified as having filarial lymphedema? Of those identified how many were referred to self-care and coping management sessions? How many showed up for management of self-care and coping? Additionally, outcome evaluation questions pertaining to outcomes and goals (columns 4 and 5) would include effect on determinants such as skill acquisition, behavior such as leg washing, and outcomes such as quality of life. 


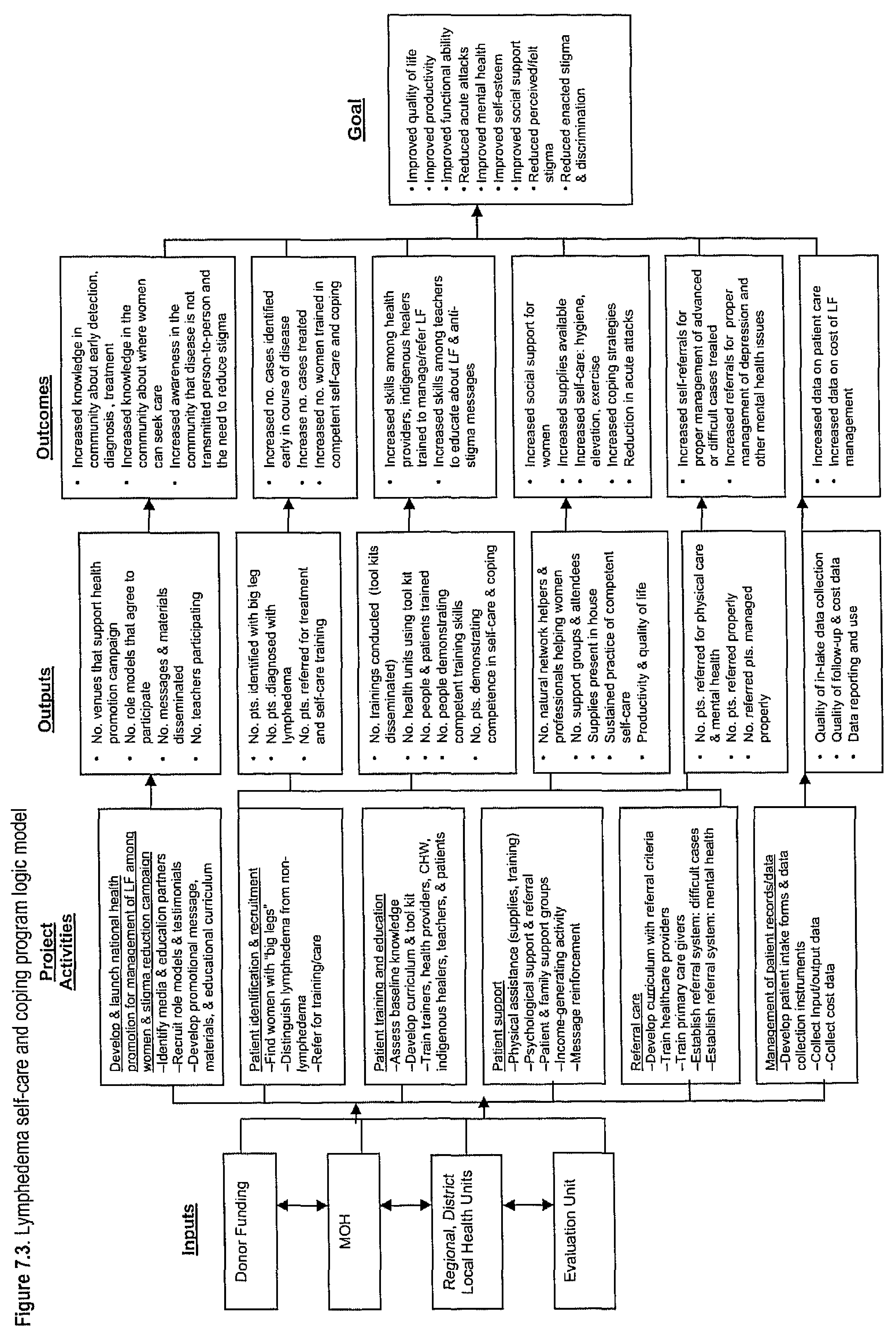


Measures of the impact of the lymphedema management intervention on determinants, behaviors and environmental conditions are derived from the matrices of change objectives. For example, looking back at the matrices in Step 1, a measure of self-efficacy could be based on the items in the self-efficacy column; a measure of skills from the skills column and so forth. The focus of the evaluation should be on specific change indicators and measures for determining whether the lymphedema management program of prescribed self-care behaviors and coping strategies among women slows progression of disease and disability, improves psychological wellbeing, and overall improves quality of life. Additionally, evaluation of environmental factors and change agent should include indicators and measures focused on successful patient instruction in efficacious self-care and coping practices provided by healthcare and public health professionals; the successful instruction of students on the transmission, prevention, diagnosis; and treatment of lymphatic filariasis and sequelae of disease provided by educators; incorporation of efficacious self-care practices with appropriate referrals for medical complications in treatments provided by indigenous healers; and training and support provided by natural helper networks.

\subsubsection{Step 6: Case Study Limitations}

The case study on evaluation presented a logic model of the program and examples of how to develop evaluation questions and measures based on the previous steps of IM. We did not present a full evaluation plan. A full plan would have included all evaluation questions, measures, a design to protect against threats to validity and a protocol for data collection.

\section{Discussion}

Lymphatic filariasis is a neglected disease across the globe. Lymphedema management programs if they exist are often under-funded to deal with basic selfcare must less psychosocial and gender specific needs. Women in our study described exhaustive attempts at seeking treatments and ultimately a cure for their lymphedema. They disclosed that lack of knowledge about their disease and effective self-care practices and coping strategies; decreased self-efficacy in their ability to change the status of their leg and the distress it imposed in their lives; lack of skills to modify the skin texture, wounds, swelling and reduce acute attacks of their legs; and no hope or expectation that their leg or life could be different were key behavioral determinants for a focused intervention.

In this paper we proposed evidence- and theory-informed recommendations developed using an IM framework for a public health intervention and program evaluation targeted to these determinants as well as specific determinants identified for their environmental change agents in filariasis endemic countries. Program recommendations based in behavioral self-regulation theories were associated with determinants, methods, and practical strategies to encourage women to consistently use effective self-care practices to manage their lymphedema on a daily basis and replace unconscious, non-useful coping strategies with useful coping strategies. In addition we proposed program components to enhance healthcare provider's ability to diagnose and manage the resulting physical and psychosocial sequelae of 
lymphatic filariasis; instruct women in efficacious self-care practices and coping practices; and promote education and stigma reduction strategies. We also recommended that indigenous healers be taught to incorporate efficacious self-care practices into their healing activities and refer complications to trained healthcare providers. To further the reach of the proposed programs we included educator and school program components to promote community education for early identification of disease and stigma reduction strategies in the schools to decrease disease-related stigma from a young age, and education and support for members of women's natural helper networks. Additional community education components included mass media activities.

The Intervention Mapping framework as we have presented allows for an evidence-based approach integrating theory with practical strategies for changing behavioral determinants of women and their change agents in real-life situations to prevent disease progression, disability, and the decline of psychosocial well-being. Our formative findings note that environmental factors, such as poverty, lack of education, gender-related power differentials, lack of housing and clean water, and access to transportation also contribute to the burden and stress women with lymphedema face. A lymphedema management program only focusing on the behavioral determinants of women may run the risk of victim-blaming and less than successful intervention outcomes. Interventions to mitigate negative environmental factors that contribute to the suffering of women must also be addressed.

The detailed IM planning process, as presented by the authors of the method, may be somewhat burdensome for program planners in resource-poor countries with high demands on their time. A community-based, participatory program planning process as suggested in this paper requires a commitment of time and resources that some countries may view as demanding to an already strained public healthcare system. But, in this paper we have attempted to simplify the process and have given examples for our readers without sacrificing the critical components necessary for an efficacious and measurable intervention, while acknowledging that flexibility and cultural adaptation is critical. We suggest that traditional lymphedema programs, historically driven by clinical care models, can be enhanced with a more critical behavior change, theory-driven approach using an IM program planning framework as described in this report.

Measures of the impact of the lymphedema management intervention on determinants, behaviors and environmental conditions are derived from the matrices of program objectives for each country. Performance objectives encourage specification of outcome measures, determinants identify specific measurable constructs, and change objectives support the development of measurement questions, scales, and indices that can be tailored to be culturally and gender specific. Process evaluation measures are derived from the description of methods, conditions, strategies and program implementation processes.

The focus of the evaluation should be on specific change indicators and measures for determining whether the lymphedema management program of prescribed self-care behaviors and coping strategies among women slows progression of disease and disability, improves psychological well-being, and overall improves quality of life. Additionally, evaluation of environmental change agents 
should include indicators and measures focused on successful patient instruction in efficacious self care and coping practices provided by healthcare providers; the successful instruction of students on the transmission, prevention, diagnosis, and treatment of lymphatic filariasis and sequelae of disease provided by educators; the incorporation of efficacious self-care practices with appropriate referrals for medical complications in treatments provided by indigenous healers; and successful instruction and support for members of women's natural helper networks. 


\section{References}

1. Molyneux DH, Hotez PJ, Fenwick A. Rapid-impact interventions: how a policy of integrated control for Africa's neglected tropical diseases could benefit the poor. PLoS Med. 2005;2:e336.

2. Franco-Paredes C, Jones D, Rodriguez-Morales A, Santos-Preciado J. Commentary: improving the health of neglected populations in Latin America. BMC Public Health. 2007; 7(1):11.

3. Durrheim D, Wynd S, Liese B, Gyapong J. Lymphatic filariasis endemicity-an indicator of poverty? [editorial]. Trop Med Int Health. 2004; 9(8):843-5.

4. US Department of Health and Human Services. Life cycle of Wuchereria bancrofti. 2007. Available at: http://www.dpd.cdc.gov/dpdx/html/imagelibrary/filariasis_il.htm. Accessed July 18, 2007.

5. Addiss DG, Brady MA. Morbidity management in the Global Programme to Eliminate Lymphatic Filariasis: a review of the scientific literature. Filaria J. 2007; 6:2.

6. World Health Organization. Bridging the gaps. Report of the director-general. Geneva: The Organization; 1995.

7. Ottesen EA, Duke BOL, Karam M, Behbehani K. Strategies and tools for the control/elimination of lymphatic filariasis. Bull World Health Organ. 1997; 75(6):491503 .

8. Michael E, Bundy DAP, Grenfell BT. Re-assessing the global prevalence and distribution of lymphatic filariasis. Parasitol. 1996; 112:409-28.

9. Molyneux DH, Zagaria N. Lymphatic filariasis elimination: progress in global programme development. Ann Trop Med Parasitol. 2002; 96(Suppl 2):S15-40.

10. Babu BV, Nayak AN, Dhal K. Epidemiology of episodic adenolymphangitis: a longitudinal prospective surveillance among a rural community endemic for bancroftian filariasis in coastal Orissa, India. BMC Public Health. 2005;5(1):50.

11. Pani SP, Yuvaraj J, Vanamail P, Dhanda V, Michael E, Grenfell BT, et al. Episodic adenolymphangitis and lymphoedema in patients with bancroftian filariasis. Trans Roy Soc Trop Med Hyg. 1995; 89:72-4.

12. World Health Organization. Annual report on lymphatic filariasis 2002. Geneva: World Health Organization; 2003.

13. Addiss DG, Louis-Charles J, Wendt JM. Epidemiology of "acute attacks" among patients in a treatment program for filariasis-associated lymphedema of the leg, Leogane, Haiti. Abstract 415. Am J Trop Med Hyg. 1999;61(Suppl 3):320.

14. Dreyer G, Medeiros Z, Netto MJ, Leal NC, de Castro LG, Piessens WF. Acute attacks in the extremities of persons living in an area endemic for bancroftian filariasis: differentiation of two syndromes. Trans R Soc Trop Med Hyg. 1999;93(4):413-7.

15. Dunyo SK, Nkrumah FK, Ahorlu CK, Simonsen PE. Exfoliative skin manifestations in acute lymphatic filariasis. Trans R Soc Trop Med Hyg. 1998;92(5):539-40.

16. Ramaiah KD, Ramu K, Kumar KN, Guyatt H. Epidemiology of acute filarial episodes caused by Wuchereria bancrofti infection in two rural villages in Tamil, Nadu, south India. Trans R Soc Trop Med Hyg. 1996;90(6):639-43.

17. Gyapong JO, Evans D, Aikins M, Gyapong M, Adjei S. The economic burden of lymphatic filariasis in northern Ghana. Ann Trop Med Parasitol. 1996;90(1):39-48.

18. Gyapong M, Gyapong JO, Adjei S, Vlassoff C, Weiss M. Filariasis in Northern Ghana: some cultural beliefs and practices and their implications for disease control. Soc Sci Med. 1996;43(2):235-42.

19. Gyapong M, Gyapong JO, Weiss M, Tanner M. The burden of hydrocele on men in Northern Ghana. Acta Tropica. 2000;77(3):287-94.

20. Ramaiah KD, Kumar KN, Ramu K, Pani SP, Das PK. Functional impairment caused by lymphatic filariasis in rural areas of south India. Trop Med Int Health. 1997;2(9):832-8.

21. Babu BV, Nayak AN, Dahl K, Acharya AS, Jangrid PK, Mallick G. The economic loss due to treatment costs and work loss to individuals with chronic lymphatic filariasis in rural communities of Orissa, India. Acta Trop. 2002;82(1):31-8. 
22. Dreyer $G$, Noroes J, Addiss D. The silent burden of sexual disability associated with lymphatic filariasis. Acta Tropica. 1997;63(1):57-60.

23. Ramaiah KD, Guyatt H, Ramu K, Vanamail P, Pani SP, Das PK. Treatment costs and loss of work time to individuals with chronic lymphatic filariasis in rural communities in south India. Trop Med Int Health. 1999 1999;4(1):19-25.

24. Suma TK, Shenoy RK, Kumaraswami V. A qualitative study of the perceptions, practices and socio-psychological suffering related to chronic brugian filariasis in Kerala, southern India. Ann Trop Med Parasitol. 2003;97(8):839-45.

25 Bandyopadhyay L. Lymphatic filariasis and the women of India. Soc Sci Med. 1996;42(10):1401-10.

26. Person B, Addiss D, Kay Bartholomew LK, Meijer C, Pou V, Gonzálvez G, et al. "Can it be that God does not remember me?": a qualitative study on the psychological distress, suffering, and coping of Dominican women with chronic filarial lymphedema and elephantiasis of the leg. Healthcare for Women International. 2008;29:349-65.

27. Person B, Addiss DG, Bartholomew LK, Meijer C, Pou V, Borne BV. Health-seeking behaviors and self-care practices of Dominican women with lymphedema of the leg: implications for lymphedema management programs. Filaria J. 2006;5(1):13.

28. Person B, Addiss D, Bartholomew LK, Meijer C, Pou V, Gonzálvez G, et al. A qualitative study of the psychosocial and health consequences associated with lymphedema among women in the Dominican Republic. Acta Tropica. 2007;103(2):90-7.

29. Person B, Bartholomew LK, Addiss D, Borne van den B. Disrupted social connectedness among Dominican women with chronic filarial lymphedema. Patient Educ Couns. 2007; 68(3):279-86.

30. World Health Organization. The World Health Report 1998. Geneva: World Health Organization; 1998.

31. Seim AR, Dreyer G, Addiss DG. Controlling morbidity and interrupting transmission: twin pillars of lymphatic filariasis elimination. Revista de Sociedade Brasileira de Medicina Tropical. 1999;32(3):325-8.

32. Dreyer G, Addiss D, Dreyer P, Noroes J. Basic lymphoedema management: treatment and prevention of problems associated with lymphatic filariasis. Hollis, NH: Hollis Publishing Company; 2002.

33. Babu BV, Nayak AN. Footcare among lymphoedema patients attending a filariasis clinic in South India: a study of knowledge and practice. Ann Trop Med Parasitol. 2003;97(3):321-4.

34. Schellekens SM, Ananthakrishnan S, Stolk WA, Habbema JDF, Ravi R. Physicians' management of filarial lymphoedema and hydrocele in Pondicherry, India. Trans R Soc Trop Med Hyg. 2005;99(1):75-7.

35. Ehrenberg JP, Ault SK. Neglected diseases of neglected populations: thinking to reshape the determinants of health in Latin America and the Caribbean. BMC Public Health. 2005;5:119.

36. Holveck JC, Ehrenberg JP, Ault SK, et al. Prevention, control, and elimination of neglected diseases in the Americas: pathways to integrated, inter-programmatic, intersectorial action for health and development. BMC Public Health. 2007;7:6.

37. Gwatkin DR, Guillot M, Heuveline, P. The burden of disease among the global poor. Lancet.1999; 354:586-89.

38. Fisher JD, Fisher W. Changing AIDS-risk behavior. Psychological Bulletin. 1992;111(3): 455-74.

39. Lyles CM, Kay LS, Crepaz N, Herbst JH, Passin WF, Kim AS, et al, Best Evidence Interventions: Findings from a systematic review of HIV behavioral interventions for US populations at high risk, 2000-2004. American Journal of Public Health. 2007;97(1):133-43.

40. Wingood GM. DiClemente RJ. HIV sexual risk reduction interventions for women. A review. American Journal of Preventive Medicine. 1996;12(3):209-17.

41. Bartholomew LK, Parcel GS, Kok G, Gottlieb NH. Planning health promotion programs. 2nd ed. San Francisco: Jossey-Bass; 2006. 
42. Alewijnse D, Mesters IEPE, Metsemakers JFM, Van den Borne B. Program development for promoting adherence during and after exercise therapy for urinary incontinence. Patient Educ Couns. 2002;48(2):147-60.

43. Bartholomew LK, Gold RS, Parcel GS, Czyzewski DI, Sockrider MM, Fernandez M, et al. Watch, Discover, Think, and Act: evaluation of computer-assisted instruction to improve asthma self-management in inner-city children. Patient Educ Couns. 2000;39(23):269-80.

44. Heinen MM, Bartholomew LK, Wensing $M$, van de Kerkhof $P$, van Achterberg T. Supporting adherence and healthy lifestyles in leg ulcer patients: systematic development of the Lively Legs program for dermatology outpatient clinics. Patient Educ Couns. 2006;61(2):279-91.

45. Bartholomew LK, Sockrider MM, Seilheimer DK, Czyzewski DI, Parcel GS, Spinelli SH. Performance objectives for the self-management of cystic fibrosis. Patient Educ Couns. 1993;22: 15-25.

46. Gyapong JO. Lymphatic filariasis in Ghana: from research to control. Trans R Soc Trop Med Hyg. 2000;94(6):599-601.

47. Gyapong JO, Adjei S, Sackey SO. Descriptive epidemiology of lymphatic filariasis in Ghana. Trans R Soc Trop Med Hyg. 1996;90(1):26-30.

48. US Department of Health and Human Services. Lymphatic filariasis in the Americas: an epidemiologic history. Atlanta, GA: Centers for Disease Control and Prevention; 2002.

49. Vincent AL, Ureña Rojas CA, Ayoub EM, Ottesen EA, Harden EG. Filariasis and erisipela in Santo Domingo. J Parasitol. 1998;84(3):557-61.

50. Vincent AL, Gonzalvo A, Cowell BC, Nayar JK, Uribe L. A survey of Bancroftian filariasis in the Dominican Republic. J Parasitol. 1987;73(4):839-40.

51. Muhr T. ATLAS-ti version 4.2 [computer software]. Berlin: Scientific Software; 1997.

52. Clark NM, Gong M, Kaciroti N. A model of self-regulation for control of chronic disease. Health Educ Behav. 2001;28(6):769-82.

53. Holman $\mathrm{H}$, Mazonson $\mathrm{P}$, Lorig $\mathrm{K}$. Health education for self-management has significant early and sustained benefits in chronic arthritis. Trans Assoc Am Physicians. 1989;102:204-8.

54. van Dam HA, van der Horst FG, Knoops $\mathrm{L}$, Ryckman RM, Crebolder HF, van den Borne BH. Social support in diabetes: a systematic review of controlled intervention studies. Patient Educ Couns. 2005;59(1):1-12.

55. Paris SG, Paris AH. Classroom applications of research on self-regulated learning. Educational Psychologist. 2001;36:89-101.

56. Locke EA, Latham GP. Building a practically useful theory of goal setting and task motivation: a 35-year odyssey. American Psychologist. 2002;57(9):705-17.

57. Bandura A. Self-efficacy: the exercise of control. New York: Freeman WH; 1997.

58. Bandura A. Health promotion by social cognitive means. Health Educ Behav. 2004; 31(2):143-64.

59. Murphy DA, Rotheram-Borus MJ, Reid HM. Adolescent gender differences in HIVrelated sexual risk acts, social-cognitive factors and behavioral skills. Journal of Adolescence. 1998;21(2):197-208.

6o. Shegog R, Bartholomew LK, Parcel GS, Sockrider MM, Mâsse L, Abramson SL. Impact of a computer-assisted education program on factors related to asthma self-management behavior. Journal of the American Medical Informatics Association. 2001;8:4961.

61. Clark NM, Zimmerman BJ.A Social cognitive view of self-regulated learning about health. Health Education Research. 1990;5(3):371-9. 


\section{Samenvatting}

Het doel van het onderzoek dat is beschreven in dit proefschrift, is het beter begrijpen van het effect van chronische lymphedema op de levens van vrouwen in ontwikkelingslanden waar lymphatic filariasis endemisch is. Lymphatic filariasis is een van de 13 parasitaire en bacteriële tropische infectieziekten waaraan weinig aandacht wordt besteed. Lymphatic filariasis wordt veroorzaakt door nematodes (rondwormen) die leven in de lymfatische vaten en het bloed van mensen. Deze door muskieten overgedragen parasitaire ziekte heeft meer dan 120 miljoen mensen aangetast en is een van de voornaamste oorzaken van permanente invaliditeit. De ziekte komt met name voor in de tropische en subtropische gebieden van Afrika, Azië en Amerika, waaronder de Pacific Islands en wordt beschouwd als een ziekte van de armen. Lymphatic filariasis heeft directe en indirecte fysieke en psychosociale invloeden op het leven van mensen die aan deze ziekte lijden en resulteert in stigmatisering, verminderde productiviteit en toegenomen medische kosten die weer leiden tot nog grotere armoede.

Lymphatic filariasis is een infectieziekte die zich uit in een serie klinische manifestaties variërend van a-symptomatische schade aan het lymfatische systeem tot chronische lymphedema van een hele arm of been, vulva of borst als gevolg van terugkerende aanvallen van acute ontsteking van de huid, lymfatische vaten en knopen, of ledematen die veroorzaakt worden door bacteriële of schimmelinfecties. Veranderingen van de huid komen veel voor bij de aangetaste ledematen en betreffen misvormende plooien in de huid, verharde huidlagen, pigmentveranderingen, abnormale verdikking van de weefsels, chronische zweren, knobbels op en onder de huid, haargroei, en ontsteking van de huid in de huidplooien. Schimmelinfecties komen ook veel voor. Elefantiasis is de ernstigste vorm van chronische lymphedema met als kenmerken ernstige ontsteking van het bindweefsel of verdikking van de huid waardoor deze lijkt op de huid van een olifant.

Slechts een paar onderzoeken hebben zich tot nog toe gericht op de gevolgen van lymphatic filariasis op de kwaliteit van leven van geinfecteerde vrouwen. De onderzoeken gepresenteerd in deze dissertatie, zijn gebaseerd op kwalitatief onderzoek van 2 jaar naar de kwaliteit van leven van vrouwen met lymphatic filariasis in de zuidelijke kuststreek van de Dominicaanse Republiek en in rurale vissersdorpen en landbouwgemeenschappen in het zuidelijk kustgebied van de Western and Central regions van Ghana in West Afrika waar filariasis endemisch is. Ongeveer 740.000 mensen in de Dominicaanse Republiek en bijna de helft van de bevolking van Ghana, 10.2 miljoen mensen, wonen in gebieden waar lymphatic filariasis endemisch is, waardoor zij risico lopen op besmetting. Tengevolge van onvoorziene omstandigheden konden de onderzoeksgegevens uit Ghana alleen betrokken worden bij de analyses van de stigmatiserende effecten van lymphatic filariasis (hoofdstuk 6) en bij de ontwikkeling van een model voor een interventieprogramma voor vrouwen met lymphatic filariasis (hoofdstuk 7).

Een grounded theory benadering van de data analyse van 6

focusgroepdiscussies en 52 key informant interviews resulteerde in 5 belangrijke thema's die uit de data naar voren kwamen: 1) de gevolgen van lymphedema voor de gezondheid en de psychosociale gevolgen; 2) de gevolgen van lymphedema voor de psychische gezondheid; 3) het gezondheidsgedrag en zelfzorggedrag van vrouwen; 4) de rol van sociale steun in het omgaan met de ziekte en de zorg; en 5) de ervaring van vrouwen met betrekking tot aan gezondheid gerelateerde stigma's. De uitkomsten verschaften aanwijzingen voor de ontwikkeling van mede op theorie gebaseerde 
aanbevelingen voor een programma voor vroege ontdekking van de ziekte, preventie van ziekteprogressie en het managen van de medische gevolgen en de gevolgen voor het verloop van de ziekte en de daaruit voortkomende invaliditeit bij vrouwen in ontwikkelingslanden waar lymphatic filariasis endemisch is.

Hoofdstuk 2 geeft een overzicht van de gevolgen voor de gezondheid en de psychosociale gevolgen die samenhangen met lymphedema zoals deze gerapporteerd werden door de Dominicaanse vrouwen in het onderzoek. De vrouwen gaven een eigen verklaring voor hun ziekte waarbij zij hun symptomen beoordeelden en interpreteerden naar aanleiding van hun ervaringen en de contextuele factoren van de eigen cultuur en hun dagelijks leven. De vrouwen beschreven een eerste probleemoplossende fase van de ziekte waarin familie en vrienden fungeerden als leken-gezondheidsadviseurs met verwijzingen naar gebedsgenezers, wonderdokters en tovenaars. Verder onderzoek hiervan zoals nader beschreven in hoofdstuk 4, geeft aan dat familie, vrienden en de sterk cultureel bepaalde overtuigingen invloed hadden op de eigen uitleg van de ziekte door de vrouwen en dat dit hun gedrag met betrekking tot het zoeken naar gezondheid sterk beïnvloedde. Deze gegevens werden verwerkt in een verklaringsmodel vanuit het perspectief van de patiënten, het explanatory model of illness (EMS). Lichamelijke en functionele beperkingen werden niet altijd in verband gebracht met de ernst van de symptomen of, zoals beschreven in dit onderzoek, het stadium van lymphedema. Ondanks de functionele beperkingen en psychische gevolgen beschouwden veel Dominicaanse vrouwen zichzelf niet als gehandicapt. Het meest opvallende gevolg dat door de Dominicaanse vrouwen beschreven werd, was de algemene ervaring van psychische stress tijdens alle stadia van de ziekte. Hierop wordt uitgebreid ingegaan in hoofdstuk 3. Terwijl de gevolgen van de ziekte vaak resulteerden in een verandering of verlies van sociale rollen, gingen sommige vrouwen met ernstige lymphedema door met zorgen voor zichzelf en hun families en met activiteiten die inkomen genereerden en zij maakten zelfs plannen voor nieuwe activiteiten. Religieuze overtuigingen, een positieve kijk op het leven en hoop vormden de basis voor positieve toekomstverwachtingen terwijl ouder worden, vermoeidheid en gevoelens van hopeloosheid de negatieve toekomstverwachtingen versterkten.

In hoofdstuk 3 wordt beschreven hoe met gebruikmaking van aangepaste principes van de grounded theory methoden de psychische toestand van de Dominicaanse vrouwen werd onderzocht en de copingstrategieën die zij gebruikten om de nadelige psychische effecten van deze ziekte te verminderen. Hoewel er individuele verschillen waren, beschreven veel vrouwen gevoelens van schaamte, verdriet, depressiviteit en angst. Daarnaast ervaarden veel vrouwen medelijden, sociale afzondering en stigmatisering. Dit leidde bij sommige vrouwen tot wanhoop en gedachten aan zelfmoord. Vrouwen waren zich sterk bewust van en schaamden zich over hoe hun benen eruitzagen. Omdat zij geen schoenen konden dragen, weerhield hen dit van het bijwonen van sociale activiteiten en het vervullen van de sociale rollen en verantwoordelijkheden die in hun cultuur van hen verwacht werden. De misvorming van de benen had bij veel vrouwen ook een negatieve invloed op het eigen lichaamsbeeld, op het zelfvertrouwen en op de sociale identiteit en het was een bron van stigmatisering, waarop dieper ingegaan wordt in hoofdstuk 6 . Het onvermogen om te voldoen aan de traditionele culturele rollen van de vrouw als echtgenote, moeder en opvoeder droeg vaak bij aan het zich sociaal geïsoleerd voelen van vrouwen, hetgeen weer leidde tot een verlies van zin in het leven en een verminderd gevoel van eigenwaarde. Psychische stress was algemeen in de levens van de vrouwen en de ernst en het type stress bleek te variëren met het verloop van de tijd. Ouder worden, samen met psychische stress, verstoorde sociale 
verbondenheid en het verergeren van de ziekte bleek te leiden tot een hoogst kwetsbare groep van vrouwen die diepgaander worden beschreven in hoofdstuk 5 . Eigen mogelijkheden en copingstrategieën en externe hulpbronnen als geld, onderwijs en sociale steun bleken van invloed te zijn op het vermogen tot omgaan met de ziekte. De vrouwen beschreven ook betekenisvolle ervaringen en adaptieve copingstrategieën voor het omgaan met hun stress. De vrouwen gebruikten probleemoplossende copingstrategieën waaruit bleek dat zij intensief en doelgericht zochten naar zorg voor hun benen. Zij gebruikten ook emotionele copingstrategieën waarbij zij familie, vrienden en sociale netwerken uitzochten om om te gaan met de emotionele gevolgen van de ziekte. Door downward social comparison konden sommige vrouwen hun situatie positief bekijken, terwijl zij door sociaal afstand te houden op veilige afstand konden blijven van stressvolle situaties. Geloof en religie waren vaak belangrijke krachten in de copingstrategieën van de vrouwen waarbij zij bleven hopen op een wonder.

In Hoofdstuk 4 wordt het gezondheidsgedrag en het zelfzorggedrag van Dominicaanse vrouwen beschreven. Zoals eerder opgemerkt, werden de ervaringen van vrouwen, hun begrip van hun eigen ziekte en hun gezondheidsgedrag sterk bepaald door culturele beliefs en door familie en vrienden. Traditionele zorgverleners en inheemse genezers speelden voor veel vrouwen een wezenlijke rol in de lichamelijke, psychische en spirituele zorg. Traditionele genezers raadden voor lymphedema vaak specifieke behandelmethoden aan, zoals bezweringen, algemene drankjes, zalfjes, injecties en gebeden. Het gebruikelijke culturele paradigma van het eerst zoeken van behandeling door inheemse genezers, resulteerde vaak niet alleen in een belangrijke vertraging van een adequate medische behandeling maar ook in potentieel schadelijke behandelingen die de aandoening kunnen verergeren. Het was vaak slechts het mislukken van deze traditionele behandelmethoden dat ertoe leidde dat vrouwen hulp zochten bij professionele zorgverleners. Zelfs wanneer ze hulp zochten bij een arts werden de vrouwen vaak verkeerd gediagnosticeerd en werden zij maar in beperkte mate geholpen. Het niet goed begrijpen van de ziekte, de hoge kosten van gezondheidszorg en het vaak niet goed diagnosticeren door artsen leidden er bij veel vrouwen toe dat zij uiteindelijk zichzelf antibiotica injecteerden en orale pijnstillers namen zonder verder nog een arts te consulteren.

In Hoofdstuk 5 wordt de rol van sociale steun aan de vrouwen bij het omgaan met hun ziekte behandeld en de zorg die zij hierbij ontvingen. Het tijdens dit onderzoek ontwikkelde verklaringsmodel van de verstoorde sociale verbondenheid werd afgeleid uit de verklaringen van de Dominicaanse vrouwen over het nut en de afbraak van hun sociale relaties en verklaarde de primaire processen die het verbreken van de sociale samenhang veroorzaakten, waaronder het verergeren van de ziekte, de mislukte pogingen van vrouwen om zich te houden aan de culturele regels, overbelaste bronnen van sociale steun, gespannen sociale verhoudingen, beschadigde sociale rollen en de gevolgen van ouder worden. De rijkdom van hun verhalen stelde ons in staat om een veel gedetailleerder beeld te vormen van de effecten van lymphedema op sociale verbondenheid dan tot voorheen is gerapporteerd. De uitkomsten belichten de complexiteit van het leven en de moeilijkheden van het omgaan met de ziekte van deze chronisch zieke vrouwen met lymphedema die beperkte levens leiden, die een slecht zelfbeeld hebben en in sociale afzondering leven. Steun van familie en vrienden, speciaal van andere vrouwen, en steun van de kerk werden bijzonder gewaardeerd door de vrouwen. De chronische lymphedema, het ouder worden en gehandicapt zijn, de verminderde sociale contacten, samen met andere medische problemen leidde tot afzonderingsgedrag en verminderd gevoel van eigenwaarde en bleek bij te dragen aan het ontstaan van 
depressieve symptomen bij vrouwen waardoor de kwaliteit van hun leven verminderde. De uitkomsten met betrekking tot verstoorde sociale verbondenheid zoals die uit onze data naar voren kwamen, omvatten verstoorde sociale steun, slechte gezondheid, verslechterd zelfbeeld, psychische stress, symptomen van depressiviteit en sociale isolatie.

De ervaringen van vrouwen met betrekking tot stigma's gerelateerd aan hun gezondheid, worden beschreven in hoofdstuk 6 . De gedeeltelijk samenvallende concepten van enacted, perceived en internalized stigma's verschaften een kader voor het in kaart brengen van een proces van schadelijke stigmatisering ervaren door vrouwen in de Dominicaanse republiek en in Ghana. Vrouwen in beide landen beschreven de angst voor besmetting en het persoonlijk ongemak als gevolg van de geur, de zweren en de misvorming van hun benen als de belangrijkste factoren voor stigmatisering door anderen. Enacted stigma's resulteerden vaak in een verlies van inkomen, verlies van sociale identiteit, verminderde sociale interacties, verminderde toegang tot hulpbronnen en toenemende stigmatisering. Deze stigmatiserende ervaringen leidden vaak tot perceived en internalized stigma's die bijdroegen aan het toenemen van angst, depressie en lijden, hetgeen samenging met een versterkt terugtrekken in zichzelf, zelfvernedering en ziekte rolgedrag, een beschadigd lichaamsbeeld en in sommige gevallen gedachten aan zelfmoord. Vrouwen beschreven zowel positieve als negatieve strategieën om met hun ziekte om te gaan. Deze werden beïnvloed door persoonlijke en sociale factoren die bijdroegen aan negatieve gevolgen voor hun gezondheid en psychosociaal welbevinden en die samenhingen met het moeten leven met deze aandoening. De Ghanese vrouwen noemden hun onvermogen om te werken en bij te dragen aan de gemeenschap vaak als hun grootste verlies. De Dominicaanse vrouwen beschreven het niet meer hebben van het gevoel een normaal uiterlijk te hebben en het niet meer kunnen functioneren in de sociale gemeenschap als hun grootste verlies. Naar aanleiding van de resultaten van het formatieve onderzoek kan worden geconcludeerd dat de 3 deels overlappende vormen van stigma's herkenbaar zijn in de beide culturen en de problemen versterken die vrouwen ervaren als de ziekte verergert, hulpbronnen afnemen en de vrouwen ouder worden.

In Hoofdstuk 7 wordt Intervention Mapping (IM), een systematisch planning- en evaluatiemodel, gepresenteerd. In het IM proces werden de onderzoeksresultaten van de vrouwen, hun familieleden, public health professionals, werkers in de gezondheidszorg en traditionele genezers geïntegreerd. Het proces van Intervention Mapping wordt geillustreerd door middel van het gebruiken van voorbeelden van resultaten samen met manieren om relevante groepen in een participatieproces te betrekken bij elke stap om op evidence en theorie gebaseerde aanbevelingen op te stellen voor programma's om het zelfmanagement te verbeteren van filarial lymphedema.

Als overdracht van de ziekte vanaf vandaag geheel zou stoppen, zouden er in de komende jaren nog steeds duizenden mensen zijn die leven met de gevolgen van lymphatic filariasis voor hun gezondheid en psychosociaal welbevinden.

Beperkte fondsen voor programma's voor het omgaan met lymphedema vereisen dat programma's voor gezondheidsvoorlichting en gezondheidsbevordering doeltreffend zijn door het gebruiken van multiple theoretische en praktische benaderingen die bewezen hebben te werken. Het raamwerk van Intervention Mapping zoals wij het hebben laten zien, maakt een problem based benadering op maat mogelijk die de theorie integreert met praktische strategieën om gedrag en omgevingsfactoren te veranderen met als doel het voorkomen van het verergeren van de ziekte en de handicap en het voorkomen van verdere misvormingen. 


\section{Acknowledgements}

Funding for this study was provided by grant no. G401R103 from the Office of Minority and Women's Health in the National Center for Infectious Diseases, at the Centers for Disease Control and Prevention, Health and Human Services, in Atlanta, Georgia USA.

I want to begin by acknowledging all the women and their families who took the time to talk with me and share their stories. I am forever indebted.

To Meredith Hickson, whose commitment to the importance of this research and the completion of this $\mathrm{PhD}$ was unwavering, I thank you. Your steadfast belief in my abilities and your sense of humor were critical to my success.

To Dr. David Addiss I have heartfelt gratitude for introducing me to this neglected tropical disease and all the people affected by this condition. As mentor, scientist, scholar, and friend your guidance, patience, and kindness have been unlimited. You are truly a person of compassion.

To Cecelia Meijer, my translator, research assistant, and adopted family member, I could not have conducted this research without your assistance, dedication, and friendship. We will always have many stories to tell.

To Dr. Guillermo Gonzálvez who allowed me to work through the Centro Para el Control de Enfermedades Tropicales in Santo Domingo and Dr. Victor Pou from Instituto Dermatologico y Cirugia de Piel Dr. Huberto Bogaert Díaz (DHBD) who changes the lives of women with lymphatic filariasis, I extend unending gratitude for your support. I also want to thank Professor Onofre de La Rosa for his interviewing assistance, Dr. Paulino, Dr. Polibio, and all others from the Dominican Republic who contributed to this study.

To Dr. Margaret Gyapong from the Ministry of Health of Ghana and all those who supported me by interviewing, transcribing and assisting in all the work in Ghana. I extend sincere appreciation for your time, support, and dedication.

I also want to thank Dr. Eric Otteson, Director of the Lymphatic Filariasis Support Center at Emory University, and Dr. Ann Haddix, currently at the Centers for Disease Control and Prevention, for all the support and assistance they provided.

To Shannon O'Connor, I am so grateful for her time and expertise in editing and formatting my work and constructing my book.

To Bart and Kay, as mentors, scholars, and friends your support, guidance and unending commitment to my work over the years are beyond words. My heart is so grateful!

And finally but most importantly, I want to thank my husband Adam Wolkon and my daughter Mara Karell for their undying love and care during this $\mathrm{PhD}$ research. I could not have completed this without your willingness to have me travel the world, monopolize the office in our house, and bear with me during times that must have been exasperating to you both. I love you both. 


\section{Biography}

Bobbie Person was born in the United States. She attended the Medical College of Virginia, Virginia Commonwealth University in Richmond, Virginia, and obtained a degree in Occupational Therapy with a minor in Psychology. As an Occupational Therapist she specialized in Hand Rehabilitation and worked in the USA, India, Nepal and Jamaica. In 1989, she obtained a Masters of Public Health from the Emory University School of Medicine in Atlanta, Georgia, and began work with the Centers for Disease Control and Prevention, where she continues to work today in. As a Senior Behavioral Scientist at the Centers for Disease Control and Prevention, she has conducted and continues to conduct operational behavioral research in domestic and international arenas on HIV/AIDS prevention, antimicrobial resistance, hanta virus, SARS, cholera, and other endemic and epidemic infectious diseases. 